\title{
LINEAR STABILITY ANALYSIS OF EVAPORATING FALLING LIQUID FILMS
}

\author{
A THESIS SUBMITTED TO \\ THE GRADUATE SCHOOL OF ENGINEERING AND SCIENCE \\ OF BILKENT UNIVERSITY \\ IN PARTIAL FULFILLMENT OF THE REQUIREMENTS FOR \\ THE DEGREE OF \\ MASTER OF SCIENCE \\ IN \\ MECHANICAL ENGINEERING
}

By

Hammam Mohamed

August 2019 
Linear Stability Analysis of Evaporating Falling Liquid Films

By Hammam Mohamed

August 2019

We certify that we have read this thesis and that in our opinion it is fully adequate, in scope and in quality, as a thesis for the degree of Master of Science.

Luca Biancofiore(Advisor)

Metin Muradoglu

E. Yegân Erdem

Approved for the Graduate School of Engineering and Science:

Ezhan Karaşan

Director of the Graduate School 


\title{
ABSTRACT \\ LINEAR STABILITY ANALYSIS OF EVAPORATING FALLING LIQUID FILMS
}

\author{
Hammam Mohamed \\ M.S. in Mechanical Engineering \\ Advisor: Luca Biancofiore
}

August 2019

In order to improve our understanding of the wavy dynamics of evaporating falling liquid films, we perform a linear stability analysis using the Orr Sommerfeld (OS) eigenvalue problem. In the present work, the OS problem is extended to include two effects due to evaporation, namely, vapor recoil and mass loss. We present a numerical scheme based on a Chebyshev spectral method to solve the eigenvalue problem. Moreover, we validate our model by comparing the results against the long wave theory in the region of small wavenumber and weak inertia. We also demonstrate how the long wave theory completely fails in predicting the correct behavior when the inertia is strong or the wavenumber is large. By performing a perturbation energy analysis, we confirm that the instability induced by vapor recoil (E-mode) behaves in a similar fashion to the instability due ot Marangoni effect (S-mode). Through the same analysis, we demonstrate that both the Smode and the E-mode can enhance each other.

Keywords: Temporal instability, falling liquid films, phase change, OrrSommerfeld eigenvalue problem. 


\title{
ÖZET \\ BUHARLAŞAN-DÜŞEN SIVI FILMLERIN DOĞRUSAL KARARLILIK ANALIZI
}

\author{
Hammam Mohamed \\ Makine Mühendisliği, Yüksek Lisans \\ Tez Danışmanı: Luca Biancofiore
}

Ağustos 2019

\begin{abstract}
Buharlaşan-düşen sıvı filmlerin dalgalı dinamiklerini Orr Sommerfeld (OS) özdeğer problemini kullanarak doğrusal bir stabilite (kararlılık) analiziyle inceledik. Bu çalışmada, OS sorunu, buharlaşmanın buhar geri tepmesi ve kütle kaybı etkilerini de kapsayacak şekilde genişletildi. Özdeğer problemini çözmek için Chebyshev spektral yöntemine dayanan sayısal bir şema sunduk. Sonuçları uzun dalga teorisinin küçük dalga sayımı ve zayıf atalet koşullarında karşılaştırarak doğruladık. Ayrıca, uzun dalga teorisinin, atalet güçlü olduğunda veya dalga boyu büyük olduğunda doğru davranışı tahmin etmekte tamamen başarısız olduğunu gösterdik. Bir sapma enerji analizi yaparak, buhar geri tepmesi (Emodu) tarafından indüklenen kararsızlı̆̆ın, Marangoni etkisinden (S-modu) kaynaklanan kararsızlı̆̆a benzer şekilde davrandığını onayladık. Aynı analizle, S ve E modlarının birbirini geliştirebileceğini gösterdik.
\end{abstract}

Anahtar sözcükler: geçici kararsızlık, düşen sıvı filmler, faz değişimi, Orr Sommerfeld (OS) özdeğer problemi. 


\section{Acknowledgement}

First, I would like to thank my advisor Dr. Luca Biancofiore for his constant guidance and encouragement and also for pushing me farther than I thought I could go. I am also grateful for his support in my academic and personal matters where it would have been impossible to finish this work without it.

I wish to express my gratitude to Prof. Metin Muradoglu for co-supervising a part of this work. I also would like to thank his PhD student Mohamed Irfan for his help and input regarding the technical details.

I would like to thank my colleagues at FluidFrame lab for their constant encouragement and support. My deepest gratitude goes to my both colleague and brother Omair Mohamed who supported me unconditionally since the time he joined the group.

Special thanks to all my friends, specially Hande Aydoğmuş, Onur Vardar, Ali Sabbagh and Mohamad Albekaai for their encouragement and moral support which kept me inspired during the difficulties I went through.

Finally, and most importantly, huge thanks to my loving and supporting parents, your constant encouragement and support brought me to where I am now. 


\section{Contents}

\begin{tabular}{lll}
\hline & INTRODUCTION & 1
\end{tabular}

1.1 Motivation . . . . . . . . . . . . . . . . . 1

1.2 Mechanisms of instability in falling liquid films . . . . . . . . . . . 2

1.2.1 Surface wave instability (H-mode) . . . . . . . . . . . 2

1.2 .2 Marangoni effect (S-mode) . . . . . . . . . . . . 5

$1.2 .3 \quad$ Evaporation effect (E-mode) . . . . . . . . . . . . 7

1.3 Methodology and literature review . . . . . . . . . . . . . 8

1.4 Objective and structure of thesis . . . . . . . . . . . . 9

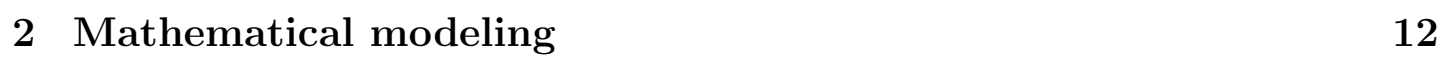

$2.1 \quad$ Governing equations and boundary conditions . . . . . . . . . . . 14

2.2 Non-dimensional and scaled parameters . . . . . . . . . . . . . 17

2.3 Base state solution . . . . . . . . . . . . . . . . . . . . 21

2.4 Long wave theory . . . . . . . . . . . . . . . . . . . . . 26 
2.4 .1 The Benney equation BE . . . . . . . . . . . 26

2.4 .2 Film base state . . . . . . . . . . . . . . . . . . 29

\begin{tabular}{|lll}
\hline 3 & Linear stability analysis: methodologies & 30
\end{tabular}

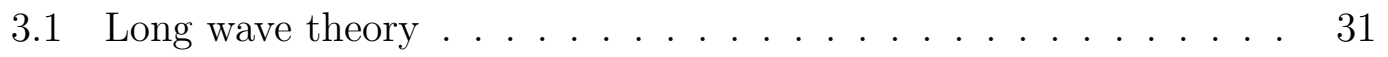

3.2 Orr-Sommerfeld eigenvalue problem . . . . . . . . . . . . . . . . . 32

$3.2 .1 \quad$ Streamwise perturbations $\left(k_{x}=k, k_{z}=0\right)$. . . . . . . 36

3.3 Perturbation energy analysis . . . . . . . . . . . . . . . . . 38

3.4 Chebyshev spectral method . . . . . . . . . . . . . . 43

3.5 Model validation . . . . . . . . . . . . . . . . . 46

3.5 .1 Non-volatile falling films . . . . . . . . . . . . . . . . 46

3.5 .2 Volatile falling films . . . . . . . . . . . . . . . . 47

$\begin{array}{|ll|}4 & \text { Linear stability analysis: } \\ 4\end{array}$

4.1 Hydrodynamic instability (H-mode) . . . . . . . . . . . . . . . . . 49

4.2 Thermocapillary instability (S-mode) . . . . . . . . . . . . . 51

4.3 Vapor recoil instability (E-mode) . . . . . . . . . . . . . . 52

4.4 Perturbation energy . . . . . . . . . . . . . . . . . . . . . . 57

$4.4 .1 \quad$ Energy analysis of the H-mode. . . . . . . . . . . . . . . 57

$4.4 .2 \quad$ Energy analysis of the S-mode . . . . . . . . . . . . 58 
$4.4 .3 \quad$ Energy analysis of the E-mode . . . . . . . . . . . . . . 59

$4.4 .4 \quad$ Energy analysis of combined S-mode and E-mode . . . . . 59

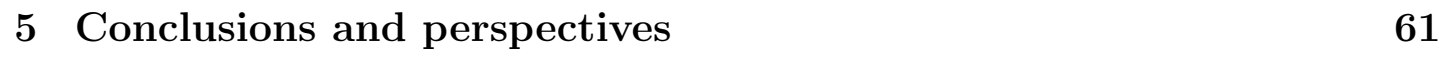

\begin{tabular}{|lll}
\hline A Interface boundary conditions of the one sided model & 69
\end{tabular}

\begin{tabular}{ll}
\hline B About the Perturbations & 71
\end{tabular}

C Direct numerical simulations (DNS) of isothermal films $\quad 74$

C.1 Numerical Solver . . . . . . . . . . . . . . . . . . . . . 74

C.2 Isothermal falling liquid film simulation . . . . . . . . . . . . . . . 76 


\section{List of Figures}

1.1 Schematic diagram of a falling film. $U$ is the fully developed viscous velocity, while $V_{c}$ is the control volume with $Q_{i n}$ the net inflow and

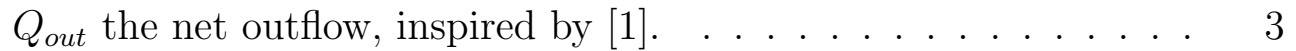

1.2 Falling film with interfacial motion induced by the effect of inertia. The dashed line corresponds to the undisturbed original interface,

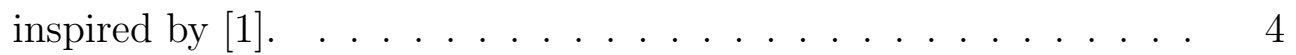

1.3 Interfacial motion due to an increase in the hydrostatic pressure under the crest. The dashed line corresponds to the undisturbed original interface, inspired by $[1] . \ldots$. . . . . . . . . . . . . . . . . 4

1.4 Mechanism of long wave instability induced by Marangoni effect (S-mode). $\quad T_{w}$ is the temperature of the wall, while $T_{\text {inf }}$ is the temperature of the ambient gas, inspired by $[1]$. . . . . . . . . 6

1.5 Mechanism of instability induced by vapor recoil effect (E-mode). $T_{w}$ is the temperature of the wall, $T_{\mathrm{inf}}$ is the temperature of the ambient gas, and $J$ is the mass flux across the interface. . . . . . 8

$2.1 \quad$ Schematic diagram of an evaporating thin film flowing down an inclined surface. . . . . . . . . . . . . . . . . . . . . . . . . . . . 13

2.2 Normal $\mathbf{n}$ and tangential $\tau_{1,2}$ unit vectors on the liquid film interface. 16 
2.3 (a) Film height, (b) Mass flux across interface, (c) Temperature gradient across the film and (d) horizontal velocity profile, for base state through time. Note that $T_{s}$ is the interface temperature, $T_{w}$ is wall temperature and $t_{D}$ is dry out time. . . . . . . . . . . . . 25

3.1 (a) Growth rate comparison between the current OS solver (solid line) and LW approximation (circles) (b) Neutral curve comparison between the current OS solver (solid line) and OS eigenvalue problem solved by Kalliadasis et al $|1|$ (circles). . . . . . . . . . . 47

3.2 Growth rate comparison between the current OS solver for $k=$ 0.001 (solid line) and LW approximation (circles) (a) for different values of $E$ vs Reynolds number and (b) for different values of $R e$ in function of time. . . . . . . . . . . . . . . . . . . . . . . . . . 48

4.1 Contours of the growth rate in the $R e-k$ plane showing the Hmode for different Kapitza number $K a$ and inclination angle $\beta$.

4.2 Contours of the growth rate in the $(R e-k)$-plane with $K a=250$, $K=1, \operatorname{Pr}=7, \beta=15^{\circ}$, and (a) $M a=0$ (b) $M a=10$ (c) $M a=20.51$

4.3 Temporal growth rate $\omega_{i}$ in versus the wavenumber $k$. Comparison is made between extended OS model (solid line) and LW expansion (dashed line) for different values of Reynolds number, when $\mathcal{V}_{r}=$ $10, K=1, \beta=15^{\circ}, K a=250, \operatorname{Pr}=7$. . . . . . . . . . . . 52

4.4 Contours of the growth rate in the $R e-k$ plane with $K a=250$, $\operatorname{Pr}=7, \beta=15^{\circ}, K=1$, and (a) $\mathcal{V}_{r}=15$ (b) $\mathcal{V}_{r}=40 \ldots . . .53$

4.5 Temporal growth rate $\omega_{i}$ in terms of the wavenumber $k$ for two different Reynolds numbers, (a) $R e=0.1$ and (b) $R e=4$. Parameters are different combinations of $\mathcal{V}_{r}$ and $M a$, while $K=1$, $\beta=15^{\circ}, K a=250, \operatorname{Pr}=7, B i=1$. . . . . . . . . . . . . . . 54 
4.6 Temporal growth rate $\omega_{i}$ in terms of the wavenumber $k$ for different values of the parameter $K$, when $R e=1, \mathcal{V}_{r}=20, \beta=15^{\circ}$, $K a=250, P r=7, B i=1$. . . . . . . . . . . . . 55

4.7 Contours of the growth rate in the $R e-k$ plane with $K a=250$, $\operatorname{Pr}=7, \beta=15^{\circ}, K=1$, and $\mathcal{V}_{r}=10$ for times: (a) $t=0 t_{d}$ (b) $t=0.33 t_{d}$ and $(\mathrm{c}) t=0.66 t_{d} \ldots \ldots . . . . . . . .56$

4.8 Normalized energy terms along $R e$ for $k=0.1, K a=250, \beta=15^{\circ} \quad 57$

4.9 Normalized energy terms along $R e$ for $k=0.1, K a=250, K=1$, $\operatorname{Pr}=7, \beta=15^{\circ}$. . . . . . . . . . . . . 58

4.10 Normalized energy terms along $R e$ for $k=0.1, K a=250, \operatorname{Pr}=7$, $\beta=15^{\circ}, M a=0, \mathcal{V}_{r}=10$, and $K=1$. . . . . . . . . . . . 59

4.11 Normalized energy terms versus $k$, for $R e=0.1, \beta=15^{\circ}, K a=$ 250, $\operatorname{Pr}=7, K=1$, (a) total energy when $M a=10$ and $\mathcal{V}_{r}=0$ (red line), $M a=0$ and $\mathcal{V}_{r}=5$ (blue line), $M a=10$ and $\mathcal{V}_{r}=5$ (yellow line). (b) $\mathrm{SHE}^{*}$, (c) MAR ${ }^{*}$, and (d)VRE $\mathrm{VRE}^{*}$. . . . . . . . 60

C.1 The standard staggered grid. The pressure nodes reside at the center of control volume. The velocity is stored at the nodes residing in the control volume edges. Picture taken from G. Tryggvason et

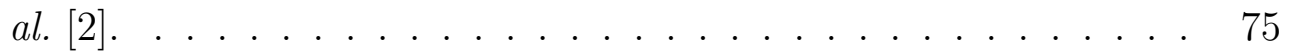

C.2 Logarithm of the root mean square velocity $\ln \left(U_{r m s}\right)$ versus time for $R e=6.5, K a=10, \beta=45^{\circ}$ and $k=0.48$. The linear growth found by our DNS (cyan line) perfectly agrees with the value of the growth rate $\left(\omega_{i}=0.034\right)$ found with our OS solver (dashed red line) while the LW theory (green line) fails to predict it. . . . . . 77 
C.3 Contours of the horizontal (a,c,e) and vertical (b,d,f) velocity at different time $(\mathrm{a}, \mathrm{b}) t=0,(\mathrm{c}, \mathrm{d}) t=22.94$ and $(\mathrm{e}, \mathrm{f}) t=77.35$. We consider an isothermal film falling down an inclined surface with $R e=6.5, K a=10, \beta=45^{\circ}$ and $k=0.48$. . . . . . . . . . . . . . 


\section{List of Tables}

2.1 Physical parameters in terms of flow rate. . . . . . . . . . . 21 


\section{Chapter 1}

\section{INTRODUCTION}

\subsection{Motivation}

Falling liquid films hold a vital position in pure and applied sciences. They are classified under the class of free-boundary problems, where highly non-linear and complex problems are encountered. Therefore, the wavy dynamics of falling liquid films have been the main focus of many researches since several decades until now. It is not only the pure science that motivates researchers to work on this topic more, but also the wide range of applications of this kind of flows, particularly in chemical processing industry. Typical examples are evaporators, heat exchangers and cooling towers in power generation. Falling films evaporators represent the state-of-art technique in the sugar industry. Moreover, they are also the basic components in water desalination plants. Aside from large scale applications, such kind of films are also used in fuel preparation systems in internal combustion engines, and also as means of thermal protection in rocket engine nozzles. Finally, they are also used in cooling of microelectronics.

With regards to heat/mass transfer applications, falling liquid films offer two main advantages (i) large contact area and small thermal resistance, and (ii)they drastically enhance heat/mass transport [3]. Some studies have found that the 
heat transfer through a wavy interface can be $10-100 \%$ higher than that of a flat film [4,5].

\subsection{Mechanisms of instability in falling liquid films}

There are many phenomenons present in falling liquid films, such as, surface waves, dry spots formation, Marangoni and evaporation effects. In order to understand how these phenomenons effect the evolution of falling films and their instability, we present the mechanisms of three different instabilities due to long surface waves, Marangoni effect, and vapor recoil.

\subsubsection{Surface wave instability (H-mode)}

Isothermal thin films falling down an inclined surface experience "long" wave deformations at the interface as shown in figure 1.1. Saying "long" means that the deformations wavelength is much longer than the film thickness. These long wave deformations are a result of the instability of an initially fully-developed flow. This instability is called the long-wave hydrodynamic instability [4], and will be referred to as the H-mode in this monograph. Three mechanisms [6] governing this instability are discussed below:

- Streamwise component of gravity:

Consider a liquid film is disturbed by adding a perturbation with a wavelength $l$ much longer than the depth of the film $\bar{h}_{N}$. The viscous parabolic profile $U$ shown in figure 1.1 is assumed to remain constant at every streamwise location as long as the height of the top surface varies slowly in the streamwise direction. Figure 1.1 shows that the net streamwise flow is positive, and increases as the depth of the film increases. Therefore, the streamwise flow is at maximum at the crest of the perturbation and, decreases to reach a minimum value at the trough. 


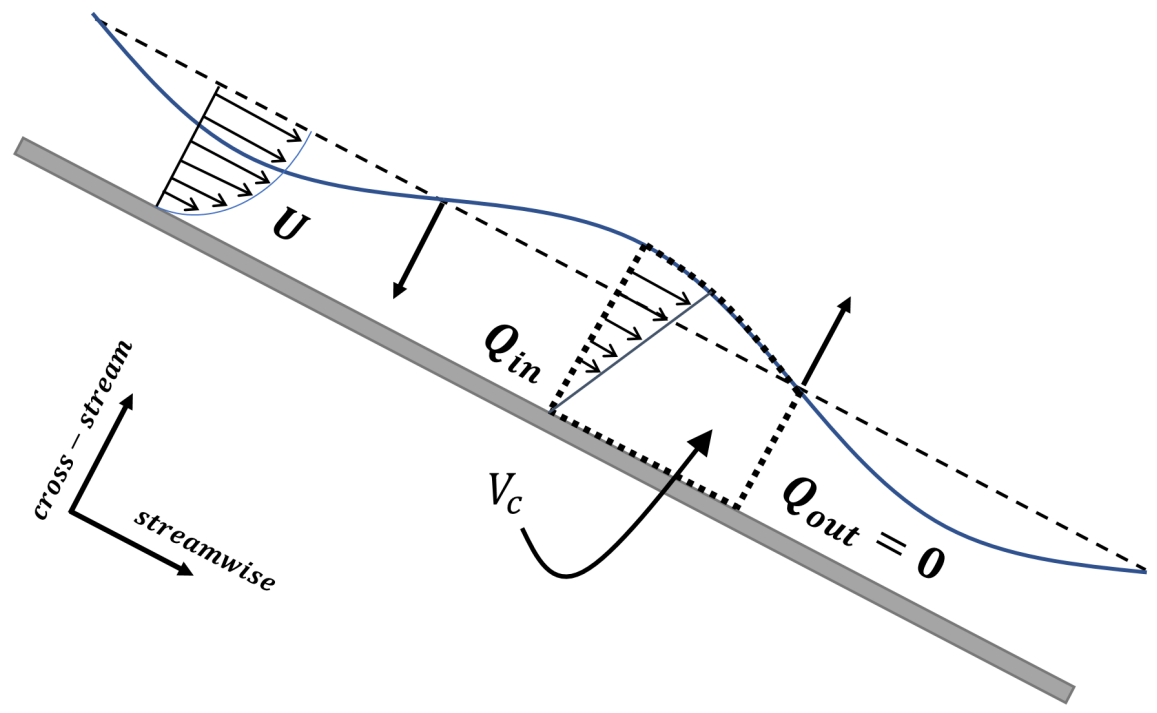

Figure 1.1: Schematic diagram of a falling film. $U$ is the fully developed viscous velocity, while $V_{c}$ is the control volume with $Q_{\text {in }}$ the net inflow and $Q_{\text {out }}$ the net outflow, inspired by [1].

Consequently, gravity will re-balance the flow rate causing the front of the crest to go upward and the rear of the crest go downward. This can be demonstrated by considering a control volume $V_{c}$ (dotted box) to the right of the perturbation crest, see figure 1.1. At the inlet there is a net flow $Q_{i n}$, however there is no net flow at the outlet $Q_{\text {out }}=0$. In order to satisfy mass conservation, the interface must go downward on the left side of the control volume, while it must go upward on the right side. These motions cause the advection of the perturbation in the streamwise direction without growth, at a phase speed higher than the velocity of any fluid particle.

\section{- Inertia:}

Figure 1.2 shows the crest of the perturbation at a particular instance of time. The surface height is increasing because of the streamwise advection of the perturbation. The fully developed velocity profile is increasing at the front face of the crest because of an increase in the interface height, while, it decreases at the rear face of the crest due to the interface height decrease. However, inertia effects prevent the flow from accelerating or decelerating fast enough to follow the fully developed velocity profile. Therefore the volume fluxes at the front and rear faces of the crest are not as the same size as the fluxes due to the fully developed film 
flow. This yields to accumulation of the flow beneath the perturbation crest and an increase in the perturbation amplitude, figure 1.2 .

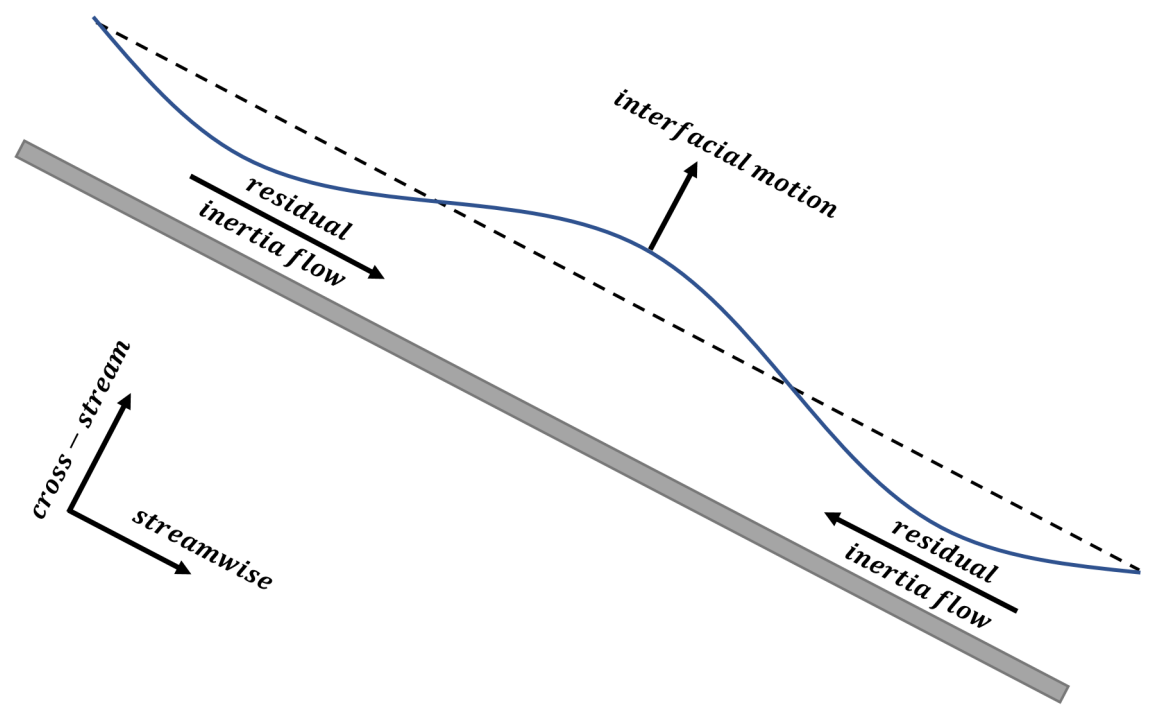

Figure 1.2: Falling film with interfacial motion induced by the effect of inertia. The dashed line corresponds to the undisturbed original interface, inspired by [1.

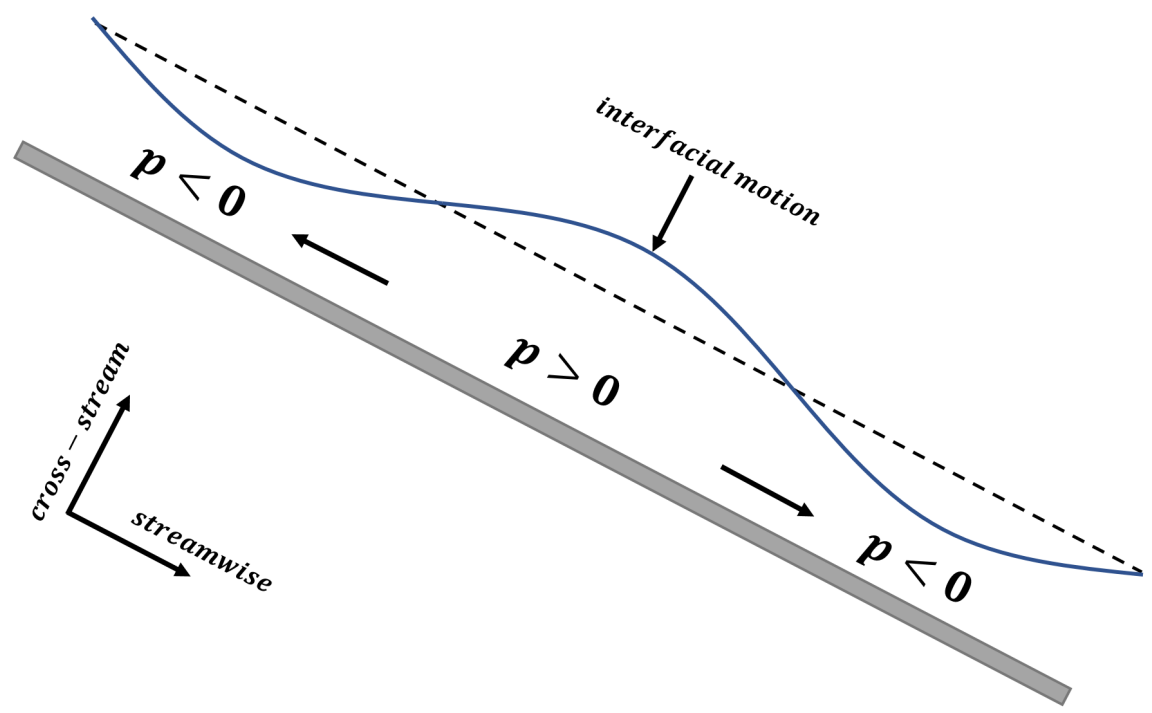

Figure 1.3: Interfacial motion due to an increase in the hydrostatic pressure under the crest. The dashed line corresponds to the undisturbed original interface, inspired by [1]. 
- Hydrostatic pressure:

The perturbation modifies the hydrostatic pressure in the film due to the gravity cross-stream component, see figure 1.3 . The hydrostatic pressure increases under the crest as the film depth increases, while it decreases under the trough where the film is thinner. In order to balance the hydrostatic pressure, the fluid is drained from under the crest and a decrease in the film height occurs, hence stabilizing the film. This effect competes against the inertia, if inertia is stronger, the film is unstable and the perturbation grows, otherwise, the film is stable and the perturbation is damped. Based on that, films falling down a vertical wall are always unstable since the hydrostatic pressure destabilizing force is canceled out [7].

Benjamin [8] studied the hydrodynamic instability of films falling down an inclined plane, and showed there exists a critical flow rate under which the uniform laminar flow could be observed, while it is not possible when the plane is vertical. He also showed that surface tension does not play an important role in deciding the critical flow rate. In conclusion, the key point in long wave hydrodynamic instability H-mode is that the perturbation travels with a phase speed much faster than any fluid particle in the flow, and that inertia plays a major role in the growth of the instability.

\subsubsection{Marangoni effect (S-mode)}

Surface tension at the interface between two fluids depends on temperature and concentration. Nonuniformity in one of these two quantities along the interface could cause the generation of a flow or changing an existing one. This behavior is called the Marangoni effect. More specifically, the effect is referred to as thermocapillary effect when there is a surface tension gradient along the interface, while it is called solutocapillary effect if the gradients are in the concentration. Only the former one will be considered in this monograph.

When surface tension is nonuniform along an interface, a flow motion is generated as a result of tangential shear stresses at the interface due to surface tension 
gradient. This gradient is a result of either two mechanisms, one is due to the modulation of the interface height, while the second is due to modifications of the temperature in the film bulk by the velocity advection. These two mechanisms yield to temperature gradient at the interface. Goussis \& Kelly [9] classified the two mechanisms as the S-mode and P-mode respectively. With regards to the later one, the P-mode results in convection patterns (rolls or hexagonal cells), where their size is of the same order as the film thickness, thus they are referred to as short-wave instabilities. One the other hand, the S-mode produces significant large-scale deformations at the interface, which is much longer than the film thickness. This instability was clearly discussed by K.A. Smith and was referred to as long-wave Marangoni instability [10]. Since we focus in this thesis on long-wave instabilities, the P-mode will not be discussed and the aftermentioned "Thermocapillary instability" or "Marangoni effect" refers to the S-mode.

\section{$\boldsymbol{T}_{\infty}$}

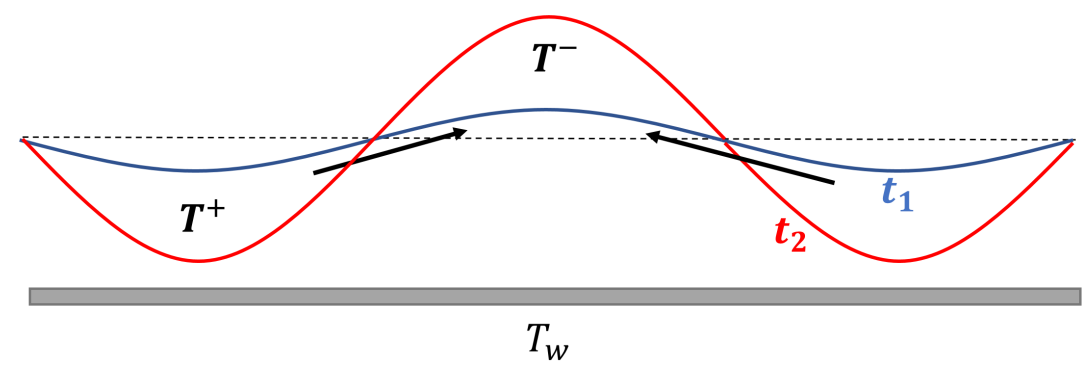

Figure 1.4: Mechanism of long wave instability induced by Marangoni effect (Smode). $T_{w}$ is the temperature of the wall, while $T_{\mathrm{inf}}$ is the temperature of the ambient gas, inspired by [1].

Now, we elaborate more on the mechanism of the long-wave Marangoni instability (S-mode), while keeping in mind that it is caused by surface temperature gradient due to interface amplitude modification, and also by assuming that surface tension decreases as temperature increases. Figure 1.4 shows a thin film on a horizontal wall heated at $T_{w}$. If an infinitesimal perturbation occurs at the interface (stage $t_{1}$ ), the temperature at the trough will be hotter than the temperature at the crest, and surface tension will be higher at the crest than that at the trough. Consequently, a flow is induced from the trough to the crest 
due to tangential stresses along the interface. This flow amplifies the infinitesimal perturbation and destabilizes the flat film (stage $t_{1}$ to stage $t_{2}$ ). The main opposing force to the long-wave Marangoni instability is the hydrostatic pressure force trying to maintain the interface flat. Moreover, for perturbations with short wave-length surface tension becomes strong and it also opposes the growth of the perturbation. For this reason instability due to S-mode takes the from of long-wave disturbances.

\subsubsection{Evaporation effect (E-mode)}

When the liquid is volatile, there is a mass flux across the interface due to phase change, where in this case we consider evaporation only. First let's assume that the evaporation rate is steady and uniform along the interface and the density of the gas is much smaller than the liquid density. When evaporation occurs at the interface, a discontinuity in the fluid velocity and the linear momentum occurs across it due to the rapid change in the fluid density. Since the vapor density is much smaller than the liquid density, the vaporized particles at the interface accelerate dramatically, causing a back reaction called vapor recoil. Vapor recoil is proportional to the evaporation rate, and also to the liquid/vapor density ratio. Figure 1.5 shows a volatile film with evaporation. When the interface is disturbed with an infinitesimal perturbation, the evaporation rate at the trough becomes higher than that at the crest, because the former has higher temperature. Consequently, the vapor recoil forces is higher at the trough than at the crest, which results in amplifying the disturbance. We named the instability due to vapor recoil as E-mode in this work. 


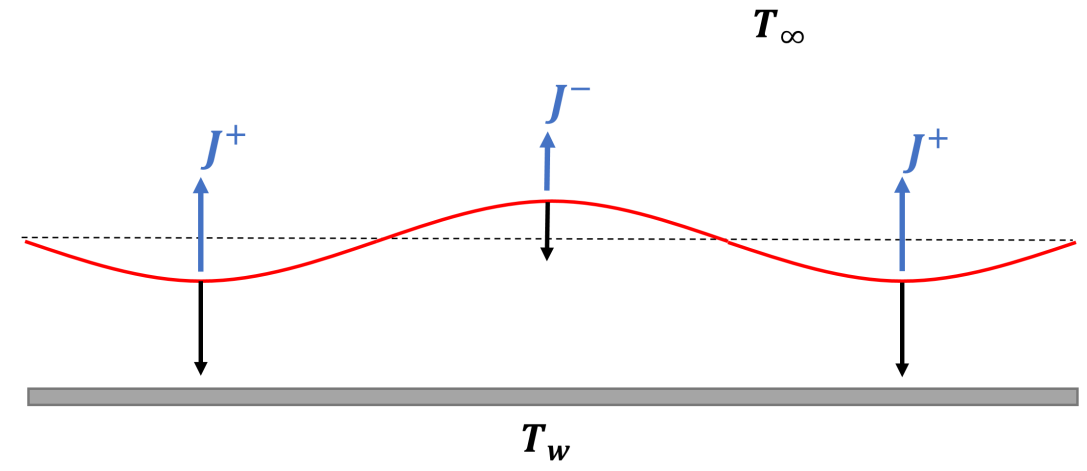

Figure 1.5: Mechanism of instability induced by vapor recoil effect (E-mode). $T_{w}$ is the temperature of the wall, $T_{\mathrm{inf}}$ is the temperature of the ambient gas, and $J$ is the mass flux across the interface.

\subsection{Methodology and literature review}

There exists several models used to study the stability falling liquid films. The governing equations and boundary conditions can be simplified to an extent depending on the flow regime under interest. As mentioned beforehand, the perturbations at the interface are much longer than the film thickness, therefore, the streamwise scale can be well separated from the cross-stream scale. The combination of the scales discrepancy and low Reynolds numbers yields to the long wave theory, where the velocity and the temperature change slowly in time. In this scenario, the flow is controlled by one single equation describing the interface evolution. This equation is named as the Benny equation (BE) referring to Benny who derived it first for isothermal films [11]. This approach was adopted by many researches afterwards to study the nonlinear dynamics of falling liquid films with different physical effects $12 \sqrt{15}]$. However, for moderate to large Reynolds numbers, the Benny equation fails, leading to a finite-time blow-up behavior [16, 17. Moreover, linear stability comparison between the Benny equation and linearized Naiver-Stokes models such as the Orr-Sommerfeld equation shows poor agreement, which degrades also as Reynolds number increases [18]. There are several models derived to overcome the setbacks of the BE, for example, the integral boundary layer approximation [19,20], the weighted residuals model [21,22]. See references [1, 12,23 for more details. 
Thermocapillary effects were considered for non-volatile heated films by Lin [24], Sreenivasan \& Lin [25], and Kelly, Davis \& Goussis [26]. They considered a falling liquid film over a uniformly heated wall and extended the BE to include the Marangoni effect. Kelly et al. showed that films under thermocapillary effect can be unstable for inclination angles well below $90^{\circ}$. Later they classified the instability into the S-mode and P-mode, and also examined the interaction between the $\mathrm{H}$-mode and S-mode showing that those instability modes enhance each other [9].

When the liquid is volatile, phase change effects were taken into consideration by Bankoff [27]. He showed that a critical Reynolds number exists for a vertical falling film under condensation effect. Furthermore, Splinder [28] concluded that the phase change term in the interfacial mass balance is only important in the case of limited wall heat flux, while for large heat fluxes, extra terms should be added to the interfacial momentum balance. Moreover, Bulerbach et al. [29] considered a horizontal volatile film, and used the long wave theory to derive a similar equation to BE, but with additional terms corresponding to thermocapillary effects, evaporation and intermolecular forces. Joo, Davis \& Bankoff [30] extended the model to include gravity effect, where they examined the nonlinear evolution of the different modes of instability. Oron [31] studied three dimensional evaporating films by solving the 3D interface evolution equation using Newton-Kantorovich method 32].

\subsection{Objective and structure of thesis}

Despite the fact that phase change process is present in many falling liquid films applications, there has not been much work related to the instabilities induced by evaporation in the literature. The main contributions are limited to approximation models, such as the long wave theory mentioned earlier. Our main objective in this work is to extend the Orr-Sommerfeld eigenvalue problem to include evaporation effects, in particular, vapor recoil and mass loss. Moreover, to examine 
the interaction between the different modes of instabilities by analyzing the perturbation energy, where this is not possible using the currently available models.

This work is structured as follows:

- In Chapter 2, the governing equations and wall boundary conditions are introduced, while the interface boundary conditions are derived using the one-sided model [29]. Then we present suitable length and time scales alongside the non-dimensional parameters that enter the evaporating falling liquid film problem, then a base state solution is obtained. Finally, we rederive the long wave theory for the scaling introduced in this work alongside the film base state.

- Chapter 3 is devoted to linear stability analysis. First, the long wave theory derived in the previous chapter is utilized to obtain analytical expressions of the growth rate and phase speed of the perturbation, the critical conditions at which the flow is unstable for different instability modes are summarized in one single expression. Moreover, the general Orr-Sommerfeld (OS) eigenvalue problem is extended to include vapor recoil and mass loss effects, which is reduced after in the limit of streamwise perturbations. Next, we extend the energy kinetic balance to include evaporation effects, where an extra term proportional to vapor recoil shows up we call it "VRE". Furthermore, we present the numerical scheme used to solve the OS eigenvalue problem. Finally we validate the extended OS model and the numerical scheme by comparing the results to different benchmarks in the literature.

- Chapter 4 presents the results of linear stability analysis performed by the Orr-Sommerfeld eigenvalue problem. We examine the different instability modes alone, and also their interactions with one another. We also analyze the perturbations energy and find the main contributing terms for every instability mode.

- Finally, chapter 5 summarizes what has been presented in this work, as well as the future plans. It also introduces a brief summary of a multiphase direct numerical simulation (DNS) solver, which was adapted to simulate 
falling liquid films. We also outline the future plan to incorporate thermal and evaporation effects into the DNS solver. 


\section{Chapter 2}

\section{Mathematical modeling}

The first objective of this chapter is to set the theoretical and mathematical ground on which this work will be built. The major assumptions necessary to formulate the problem are highlighted first. Next, the governing equations and boundary conditions are derived based on these assumptions. Moreover a suitable non-dimensionalization is made and several scaling parameters are introduced. The second objective of this chapter is to re-derive already existing models, which are necessary to perform linear stability analysis of evaporating falling liquid films.

Figure 2.1 illustrates an evaporating thin film falling down an inclined wall. The wall forms an angle $\beta$ with respect to the horizontal direction. A 3D Cartesian coordinate $(x, y, z)$ is considered, where $x$ is in the streamwise direction (direction of the flow), $y$ is the coordinate normal to the wall, and $z$ is the coordinate parallel to the cross-stream direction. The wall is fixed at $y=0$, while the interface is a function of space and time located at $y=h(x, z, t)$. The wall is uniformly heated to a fixed temperature $T_{w}$. The liquid is volatile and therefore mass flux across the interface is present $(J)$. The vapor is fixed at a constant temperature and pressure $T_{\infty}$ and $P_{\infty}$ respectively.

The local film thickness is a function of space and time $h(x, z, t)$, and $\bar{h}_{N}$ is the mean film thickness $\left(\bar{h}_{N}<1 \mathrm{~mm}\right)$, which is way shorter than a typical stream-wise 


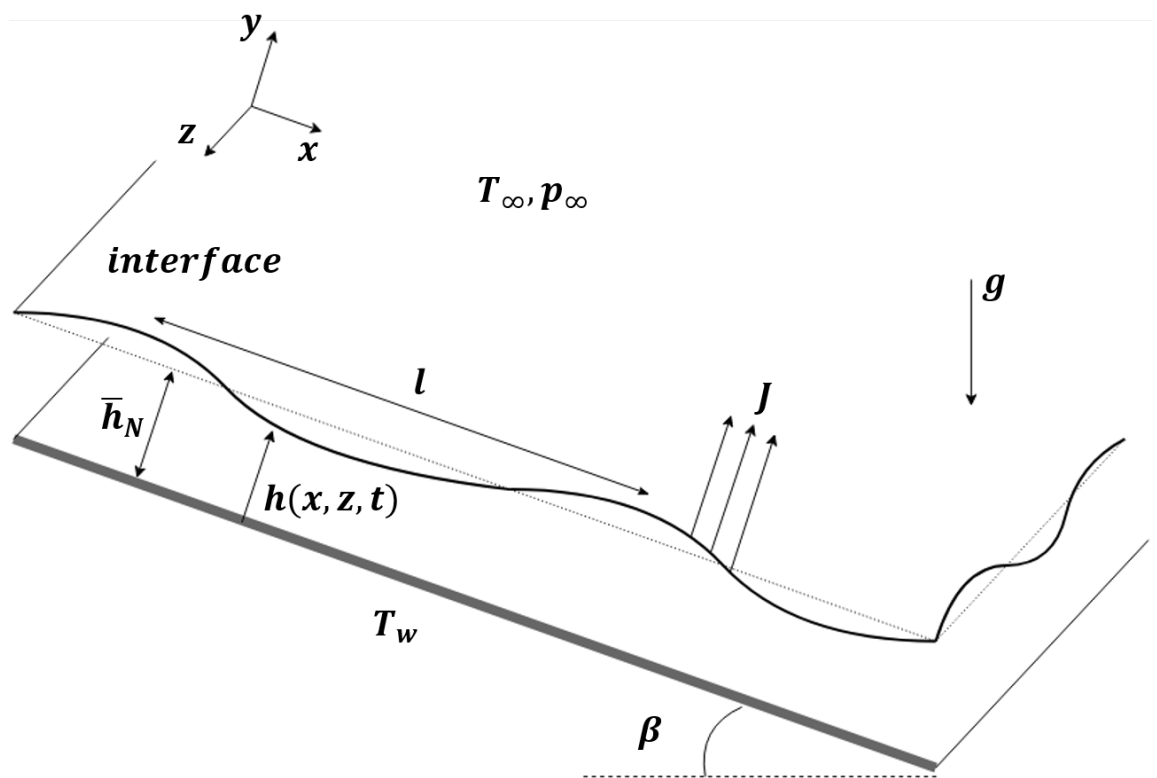

Figure 2.1: Schematic diagram of an evaporating thin film flowing down an inclined surface.

wavelength $\left(l \gg \bar{h}_{N}\right)$.

\section{The main assumptions used in this monograph are:}

- The viscosity of the liquid is constant. Additionally, all the physical parameters of the liquid are constant with respect to thermal gradients.

- The intermolecular interactions are not taken into consideration, therefore the thickness of films under focus are not in the range of $\mathrm{nm}$.

- No-penetration and no-slip boundary conditions are assumed at the wall.

- The density, viscosity and thermal conductivity of liquid are assumed to be much greater than those of the vapor, simply this says that the vapor is mechanically and thermally passive. This assumption allows decoupling the dynamics of the liquid from the dynamics of the vapor, forming what is called the "one sided model", which will be discussed in more details in the following section.

- The vapor is fixed at a constant temperature $T_{\infty}$, and thus has infinite heat capacity. It is also fixed at a constant pressure $p_{\infty}$. 
- In order to be able to model Marangoni effect, a constitutive equation that governs the relation between surface tension $\sigma\left(T_{s}\right)$ and interface temperature $T_{s}$ is needed. This can be done by expanding the surface tension using Taylor series with the reference temperature taken to be $T_{\infty}$ :

$$
\sigma=\sigma_{\infty}-\gamma\left(T_{s}-T_{\infty}\right)
$$

where $\sigma_{\infty}$ is the surface temperature at the gas temperature. The rate of surface tension change alongside temperature is $\gamma=-\left(d \sigma / d T_{s}\right), \gamma$ is assumed to be positive indicating that the surface tension decreases when the temperature of the interface increases.

- The viscous dissipation in the energy equation is neglected, this assumption is valid for the thin films and the considered thermal gradients [3].

- The evaporation rate is assumed to be steady, and proportional to the local surface temperature $\left(T_{s}\right)$ 33.

- The frozen interface assumption is utilized, which means that the viscous time scale is shorter than the evaporative scale.

\subsection{Governing equations and boundary condi- tions}

The governing equations, namely continuity, 3-D Navier-Stokes and energy for the film illustrated by figure 2.2 are:

$$
\begin{aligned}
& \partial_{y} u+\partial_{x} v+\partial_{z} w=0 \\
& \rho\left(\partial_{t} u+u \partial_{x} u+v \partial_{y} u+w \partial_{z} u\right)=-\partial_{x} p+\mu\left(\partial_{x x} u+\partial_{y y} u+\partial_{z z} u\right)+g \sin \beta \\
& \rho\left(\partial_{t} v+u \partial_{x} v+v \partial_{y} v+w \partial_{z} v\right)=-\partial_{y} p+\mu\left(\partial_{x x} v+\partial_{y y} v+\partial_{z z} v\right)-g \cos \beta \\
& \rho\left(\partial_{t} w+u \partial_{x} w+v \partial_{y} w+w \partial_{z} w\right)=-\partial_{z} p+\mu\left(\partial_{x x} w+\partial_{y y} w+\partial_{z z} w\right) \\
& \partial_{t} T+u \partial_{x} T+v \partial_{y} T+w \partial_{z} T=\frac{\kappa}{\rho c_{p}}\left(\partial_{x x} T+\partial_{y y} T+\partial_{z z} T\right)
\end{aligned}
$$


where, $u, v$ and $w$ are the fluid velocities in the directions $x, y$, and $z . p$ is pressure, $g$ is the gravitational body force, $T$ is temperature, $\kappa$ is thermal conductivity, and $c_{p}$ is constant pressure heat capacity. The wall and interface boundary conditions governing the equations (2.2) are presented below:

\section{Wall Boundary Conditions $y=0$}

- No slip boundary condition,

$$
u=v=w=0 .
$$

- The wall is heated to a constant wall temperature

$$
T=T_{w}
$$

Interface boundary conditions $y=h(x, z, t)$ :

The integral forms of the mass, momentum, energy balance laws were utilized to derive the jump conditions at the free surface of an evaporating falling film 34. Later many authors used these jump conditions to derive the "one sided model" [29 30], which states that the vapor is mechanically and thermally passive (assumption 4). The one sided model is presented directly here, while the reader is referred to appendix $\mathrm{A}$ for derivation.

- Mass balance jump condition

$$
J=\rho\left(\mathbf{v}-\mathbf{v}^{(s)}\right) \cdot \mathbf{n},
$$

with $\rho$ is the density of the liquid, $\mathbf{v}^{(s)}$ is the velocity of the interface, and $\mathbf{n}$ is the unit vector normal to the interface defined as below:

$$
\mathbf{n}=\frac{1}{n}\left(-\partial_{x} h, 1,-\partial_{z} h\right),
$$

with $\mathrm{n}=\left(1+\left(\partial_{x} h\right)^{2}+\left(\partial_{z} h\right)^{2}\right)^{1 / 2}$.

- Normal stress jump condition

$$
-\frac{J^{2}}{\rho^{(v)}}-(\mathbf{P} \cdot \mathbf{n}) \cdot \mathbf{n}=2 H \sigma(T),
$$


where, $\rho^{(v)}$ is the vapor density, $\mathbf{P}$ is the deviatoric stress tensor, and $H$ is the mean curvature of the interface defined as follows:

$$
H(h)=\frac{1}{2} \frac{\partial_{x x} h\left[1+\left(\partial_{z} h\right)^{2}\right]+\partial_{z z} h\left[1+\left(\partial_{x} h\right)^{2}\right]-2 \partial_{x} h \partial_{z} h \partial_{x z} h}{\left[1+\left(\partial_{x} h\right)^{2}+\left(\partial_{z} h\right)^{2}\right]^{3 / 2}} .
$$

Physically, the first term represent the vapor recoil, the second term is a force per unit area, and the right hand side term is the surface tension force normal to the interface. This condition states that all the normal forces must be balanced across the interface.

- Tangential stress jump condition

$$
(\mathbf{P} \cdot \mathbf{n}) \cdot \tau_{\mathbf{i}}=\nabla_{\mathbf{s}} \sigma \cdot \tau_{\mathbf{i}}, \quad i=1,2
$$

where $\nabla_{\mathbf{s}} \sigma$ is the tangential surface tension force, and $\tau_{\mathbf{i}}$ is the tangential unit vector, defined as

$$
\tau_{\mathbf{1}}=\frac{1}{\tau_{1}}\left(1, \partial_{x} h, 0\right) \quad \text { and } \quad \tau_{\mathbf{2}}=\frac{1}{\tau_{2}}\left(0, \partial_{z} h, 1\right)
$$

with $\tau_{1}=\left(1+\left(\partial_{x} h\right)^{2}\right)^{1 / 2}$ and $\tau_{2}=\left(1+\left(\partial_{z} h\right)^{2}\right)^{1 / 2}$.

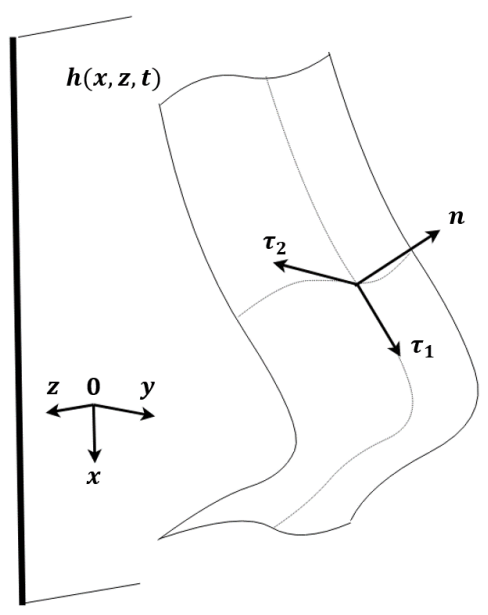

Figure 2.2: Normal $\mathbf{n}$ and tangential $\tau_{1,2}$ unit vectors on the liquid film interface.

- Energy jump condition

$$
J\left[L+\frac{1}{2}\left[\frac{J}{\rho^{(v)}}\right]^{2}\right]=-k \nabla \mathbf{T} \cdot \mathbf{n},
$$


where, and $L$ is the latent heat of vaporization. The energy jump condition states that total heat conducted across the liquid film is used either to vaporize the liquid particles at the interface (first term) or to impart kinetic energy to the vapor particles (second term).

- The linearized constitutive equation which relates the mass flux across the interface to the local interface temperature 33.

$$
J=\left(\frac{\alpha \rho^{(v)} L}{T_{v}^{\frac{3}{2}}}\right)\left(\frac{M_{w}}{2 \pi R_{g}}\right)^{\frac{1}{2}}\left(T^{(I)}-T_{\infty}\right),
$$

where, $\alpha$ is the accommodation coefficient, $\mathrm{L}$ is the latent heat, $M_{w}$ is the molecular weight, and $R_{g}$ is the universal gas constant.

\subsection{Non-dimensional and scaled parameters}

For falling liquid films, the streamwise acceleration causes the flow, while the viscosity of the liquid resists this flow, the balance between these two forces leads to a semi-parabolic velocity profile referred to as the Nusselt film solution 35. The Nusselt film solution is utilized to introduce length and time scales based on the viscous-gravity balance:

$$
l_{\nu}=\left(\frac{\nu^{2}}{g \sin \beta}\right)^{1 / 3} \text { and } \quad t_{\nu}=\left(\frac{\nu}{(g \sin \beta)^{2}}\right)^{1 / 3} .
$$

Additionally, an evaporative time scale is introduced:

$$
t_{E}=\frac{\rho \bar{h}_{N}^{2} L}{\kappa \Delta T} .
$$

It is assumed that the evaporative time scale is much longer than the viscous time scale. This leads to the frozen interface assumption which means that the evaporation is independent of the viscous forces of the flow.

These balances are utilized to scale the different parameters in the governing equations and boundary conditions $2.3-2.12$ as follows:

$$
(x, y, z)^{*} \rightarrow \bar{h}_{N}(x, y, z), \quad h^{*} \rightarrow \bar{h}_{N} h, \quad t^{*} \rightarrow \frac{t_{v} l_{v}}{\bar{h}_{N}} t
$$




$$
\begin{aligned}
\mathbf{v}^{*} \rightarrow \frac{\bar{h}_{N}^{2}}{t_{v} l_{v}} \mathbf{v}, \quad p^{*} & \rightarrow p_{\infty}+\rho \frac{l_{v} \bar{h}_{N}}{t_{v}^{2}} p, \quad T^{*} \rightarrow T_{\infty}+T \Delta T, \\
J^{*} & \rightarrow J \frac{\kappa \Delta T}{\bar{h}_{N} L},
\end{aligned}
$$

where $\Delta T=T_{w}-T_{\infty}$. Note that the starred quantities are the dimensional ones. The non-dimensional governing equations and boundary conditions are presented below:

$$
\begin{aligned}
& \partial_{x} u+\partial_{y} v+\partial_{z} w=0 \\
& 3 R e\left(\partial_{t} u+u \partial_{x} u+v \partial_{y} u+w \partial_{z} u\right)=-\partial_{x} p+\partial_{x x} u+\partial_{y y} u+\partial_{z z} u+1, \\
& 3 R e\left(\partial_{t} v+u \partial_{x} v+v \partial_{y} v+w \partial_{z} v\right)=-\partial_{y} p+\partial_{x x} v+\partial_{y y} v+\partial_{z z} v-C t \\
& 3 \operatorname{Re}\left(\partial_{t} w+u \partial_{x} w+v \partial_{y} w+w \partial_{z} w\right)=-\partial_{z} p+\partial_{x x} w+\partial_{y y} w+\partial_{z z} w \\
& 3 \operatorname{Re} \operatorname{Pr}\left(\partial_{t} T+u \partial_{x} T+v \partial_{y} T\right)=\partial_{x x} T+\partial_{y y} T+\partial_{z z} T
\end{aligned}
$$

subject to the boundary conditions, at the plate $(y=0)$ :

$$
\begin{aligned}
& u=v=w=0, \\
& T=1
\end{aligned}
$$

at the free surface $y=h(x, z, t)$ :

$$
\begin{aligned}
& v=\partial_{t} h+u \partial_{x} h+J E, \\
& p=(\mathbf{P} \cdot \mathbf{n}) \cdot \mathbf{n}-\frac{1}{n^{3}}(W e-M T) \partial_{x x} h+\frac{V_{r} J^{2}}{2}, \\
& 0=(\mathbf{P} \cdot \mathbf{n})+M\left(\partial_{x} T+\partial_{x} h \partial_{y} T\right), \\
& J+\frac{R e V_{r}}{D \mathcal{L}} J^{3}=-\nabla T . \mathbf{n}, \\
& J K=T .
\end{aligned}
$$

The parameters which govern the physical effects are presented below:

- The Kapitza number

$$
K a=\frac{\sigma_{\infty}}{\rho(g \sin \beta)^{1 / 3} \nu^{4 / 3}}
$$


the Kapitza number compares the surface tension force to the inertia. Its a function of the liquid properties and inclination angle, while independent of the flow rate.

- The Marangoni number

$$
M a=\frac{\gamma \Delta T}{\rho(g \sin \beta)^{1 / 3} \nu^{4 / 3}},
$$

Ma represents the ratio between the force resulted from the surface tension gradient across the interface and inertia.

- The Prandtl number

$$
\operatorname{Pr}=\frac{\nu \rho c_{p}}{\kappa}
$$

compares the momentum diffusivity to thermal diffusivity.

- The evaporation number

$$
\mathcal{E}=\frac{\kappa \Delta T}{\rho \nu L}
$$

measures the rate of evaporation, and compares the viscous time scale to the evaporative time scale.

- The vapor recoil number.

$$
\mathcal{V}_{r}=\frac{\mathcal{E}^{2}}{D}
$$

it is a measure of the vapor recoil, where $\left(D=3 \rho^{(v)} / \rho\right)$ is the ratio between liquid and vapor densities, where $D$ is usually very small [11]

- the latent heat number

$$
\mathcal{L}=\frac{8 \bar{h}_{N}^{2} L}{9 \nu^{2}}
$$

which is a measure of the latent heat.

Next, the non-dimensional numbers in the equations 2.13-2.15) are presented in terms of the physical parameters and the flow rate:

- The Reynolds number

$$
R e=\frac{g \sin \beta \bar{h}_{N}^{3}}{3 \nu^{2}}
$$


the Reynolds number relates the inertia to viscous forces. It also relates the dimensionless film thickness $\left(h_{N}\right)$ to the length scale:

$$
h_{N}=\frac{\bar{h}_{N}}{l_{v}}=(3 R e)^{1 / 3}
$$

- The inclination number

$$
C t=\cot \beta
$$

relates the cross stream and the stream-wise components of the gravitational force. It is a measure of the hydrostatic pressure.

- The parameter $\mathrm{K}$ from the scaled constitutive equation

$$
K=\left(\frac{\lambda T_{s}^{\frac{3}{5}}}{\alpha h_{N} \rho^{(v)} L^{2}}\right)\left(\frac{2 \pi R_{g}}{M_{w}}\right)^{0.5}
$$

The parameter $K$ measures the degree of non-equilibrium at the evaporating interface. $K=0$ corresponds to the quasi-equilibrium limit, where the interfacial temperature is constant and equal to the saturation value, while $K^{-1}=0$ corresponds to the non-volatile case in which the evaporative mass flux $(J)$ is zero.

Furthermore, When the liquid is non-volatile and the mass flux is zero $(J=$ 0 ), the energy boundary condition (2.15,f) and constitutive relation 2.15.e) are combined to form a Robin/mixed boundary condition:

$$
K \partial_{y} T+T=0
$$

this means that all the heat conducted through the liquid is dissipated into the gas at the interface, the parameter $K$ is no longer a measure of evaporation equilibrium and instead we consider $K^{-1}$ as the Biot number $B$, and we recover the base state solution of a non-volatile heated film obtained by Kalliadasis [1].

As a result of the viscous-gravity scaling, the physical parameters presented before $\left(\mathrm{Ka}, \mathrm{Ma}, \mathcal{E}\right.$ and $\left.\mathcal{V}_{r}\right)$ show up scaled with the flow rate in the non-dimensional equations 2.13-2.15), the new set of parameters are listed below: 


\begin{tabular}{lll} 
Non-Dimensional Number & Symbol & Expression \\
\hline Weber number & We & $\mathrm{Ka} /(3 R e)^{2 / 3}$ \\
Marangoni number & $\mathrm{M}$ & $\mathrm{Ma} /(3 R e)^{2 / 3}$ \\
Evaporation number & $\mathrm{E}$ & $\mathcal{E} /(3 R e)$ \\
Vapor Recoil number & $V_{r}$ & $\mathcal{V}_{r} /(R e)$ \\
\hline
\end{tabular}

Table 2.1: Physical parameters in terms of flow rate.

To elaborate more, this scaling was chosen among many others available in the literature because it relates all the physical parameters to the flow rate, and hence provide more realistic presentation of the problem. One extra advantage it that it makes comparison to experimental work accessible, since we have only Re depending on the film thickness, while all the other parameters will scale by default when Re changes.

\subsection{Base state solution}

The system of governing equations and boundary conditions (2.13-2.15) have two different solutions [29]. First solution is steady and space-dependent. The other solution is time dependent and spacially uniform representing a volatile film falling down a heated plate, while thinning at the same time due to evaporation. The later mentioned solution will be the main focus since the scope of this monograph is the temporal stability analysis. The base state quantities are specified by overbar, the equations are simplified by setting $\left(v=0, \partial_{x}=0\right)$ :

$$
\begin{aligned}
& 3 \operatorname{Re}\left(\partial_{t} \bar{u}\right)=\partial_{y y} \bar{u}+1, \\
& \partial_{y} \bar{p}=-C t, \\
& 3 \operatorname{RePr} \partial_{t} \bar{T}=\partial_{y y} \bar{T},
\end{aligned}
$$

with boundary conditions at the wall $(y=0)$ :

$$
\begin{aligned}
& \bar{u}=0, \\
& \bar{T}=1,
\end{aligned}
$$


and at the free surface $y=\bar{h}(x, t)$ :

$$
\begin{aligned}
& \partial_{t} \bar{h}=-\bar{J} E, \\
& \bar{p}=\frac{V_{r} \bar{J}^{2}}{2}, \\
& \partial_{y} \bar{u}=0 \\
& \bar{J}+\frac{R e V_{r}}{D^{-1} \mathcal{L}^{-1}} \bar{J}^{3}=-\partial_{y} \bar{T}, \\
& K \bar{J}=\bar{T} .
\end{aligned}
$$

In order to obtain the base state solution of equations $(2.17-2.19)$, it is assumed that evaporation is slow $(E<<1)$ and the system is expanded in terms of the film evaporation number $(E)[29]$. The velocity $\bar{u}(y, t)$, mass flux $\bar{J}(t)$, liquid temperature $\bar{T}(y, t)$, and pressure $\bar{p}(y, t)$ are assumed to be of order unity, while the film thickness $\bar{h}(t)$ is considered an unspecified order-one function.

$$
\begin{aligned}
& \bar{u}=u_{o}+E u_{1}+E^{2} u_{2}+\mathcal{O}\left(E^{3}\right), \\
& \bar{J}=J_{o}+E J_{1}+E^{2} J_{2}+\mathcal{O}\left(E^{3}\right), \\
& \bar{\Theta}=\Theta_{o}+E \Theta_{1}+E^{2} \Theta_{2}+\mathcal{O}\left(E^{3}\right), \\
& \bar{p}=p_{o}+E p_{1}+E^{2} p_{2}+\mathcal{O}\left(E^{3}\right) .
\end{aligned}
$$

Several approximations are used in order to find the base state solution [29]:

- Sine evaporation is slow, the effect of mass loss in the kinematic boundary condition $(2.19 a)$ is recovered by rescaling the time on the evaporation scale:

$$
t=E \tilde{t} \quad \text { and } \quad \tilde{z}=z .
$$

- The parameter $(\mathcal{L})$ is quite large [29], and therefore the kinetic energy of the vapor particles in the energy boundary condition is assumed negligible

$$
\mathcal{L}^{-1}=o(1)
$$

- The vapor recoil term in the normal stress boundary condition (2.19.b) is conserved by assuming a relationship between the small parameters $(D)$ and $(E)$

$$
D=E^{2} \bar{D}
$$


After applying the approximations and substituting the expansions in equations 2.172 .19 the leading order system is:

$$
\begin{aligned}
& \partial_{y y} u_{o}=-1, \\
& \partial_{y} p_{o}=-C t \text {, } \\
& \partial_{y y} T_{o}=0 \text {, } \\
& y=0 \text { : } \\
& u_{o}=0, \quad T_{o}=0, \\
& y=h(\tilde{t}): \\
& h_{\tilde{t}}=-J_{o}, \\
& p_{o}=\frac{V_{r} J_{o}^{2}}{2} \text {, } \\
& \partial_{y} u_{o}=0, \\
& -T_{y}=J_{o}, \\
& K J_{o}=T_{o} .
\end{aligned}
$$

with he initial condition:

$$
\tilde{t}=0: \quad \bar{h}=1 .
$$

Finally, the leading order base state solution is:

$$
\begin{aligned}
& \bar{h}(t)=-K+\left(K^{2}+2 K+1-2 E t\right)^{\frac{1}{2}}, \\
& \bar{J}(t)=\left(K^{2}+2 K+1-2 E t\right)^{-\frac{1}{2}}, \\
& \bar{U}(y, t)=y\left(h(t)-\frac{y}{2}\right), \\
& \bar{P}(y, t)=C t(h(t)-y)+\frac{V_{r}}{2} J^{2}, \\
& \bar{\Theta}(y, t)=1-J y .
\end{aligned}
$$

Figure 2.3 shows the behavior of the base state for different K values. Starting with the isothermal case $\left(K^{-1}=0\right)$, the temperature gradient across the film is zero, and therefore there is no evaporation, and the film thickness remains constant as in figure 2.3(a). The velocity keeps a parabolic profile as in figure 2.3(d). Note that the Nusselt film solution for isothermal falling films [35] can be retained by setting $(E=0, K=0)$. 
As for the non-equilibrium case $(K \neq 0)$, the film thickness decreases with time until it reaches zero at the dry out-time

$$
t_{D}=\frac{1+2 K}{2 E} .
$$

Moreover, the interface temperature $\left(T_{s}\right)$ approaches the wall temperature $\left(T_{w}\right)$ as the film thins, and the mass flux increases with time and its maximum is at the dry out time. For the quasi-equilibrium case $(K=0)$, dry out occurs within a shorter time than in non-equilibrium case.

$$
t_{D}=\frac{1}{2 E} .
$$

The temperature difference between the interface and the wall is constant, consequently the heat flux across the film increases as the film thins at higher rates than of the previous scenario $(K \neq 0)$, this explains why the mass flux is higher in this case. The velocity maintains its parabolic profile in all the three scenarios.

The base state in equation (2.21) is based on assuming that the evaporation is slow, and that the evolution of the base state in time is much slower than the perturbation growth or decaying in time. Therefore, this base state is utilized in the next chapter to study the primary instability of evaporating falling liquid films. 

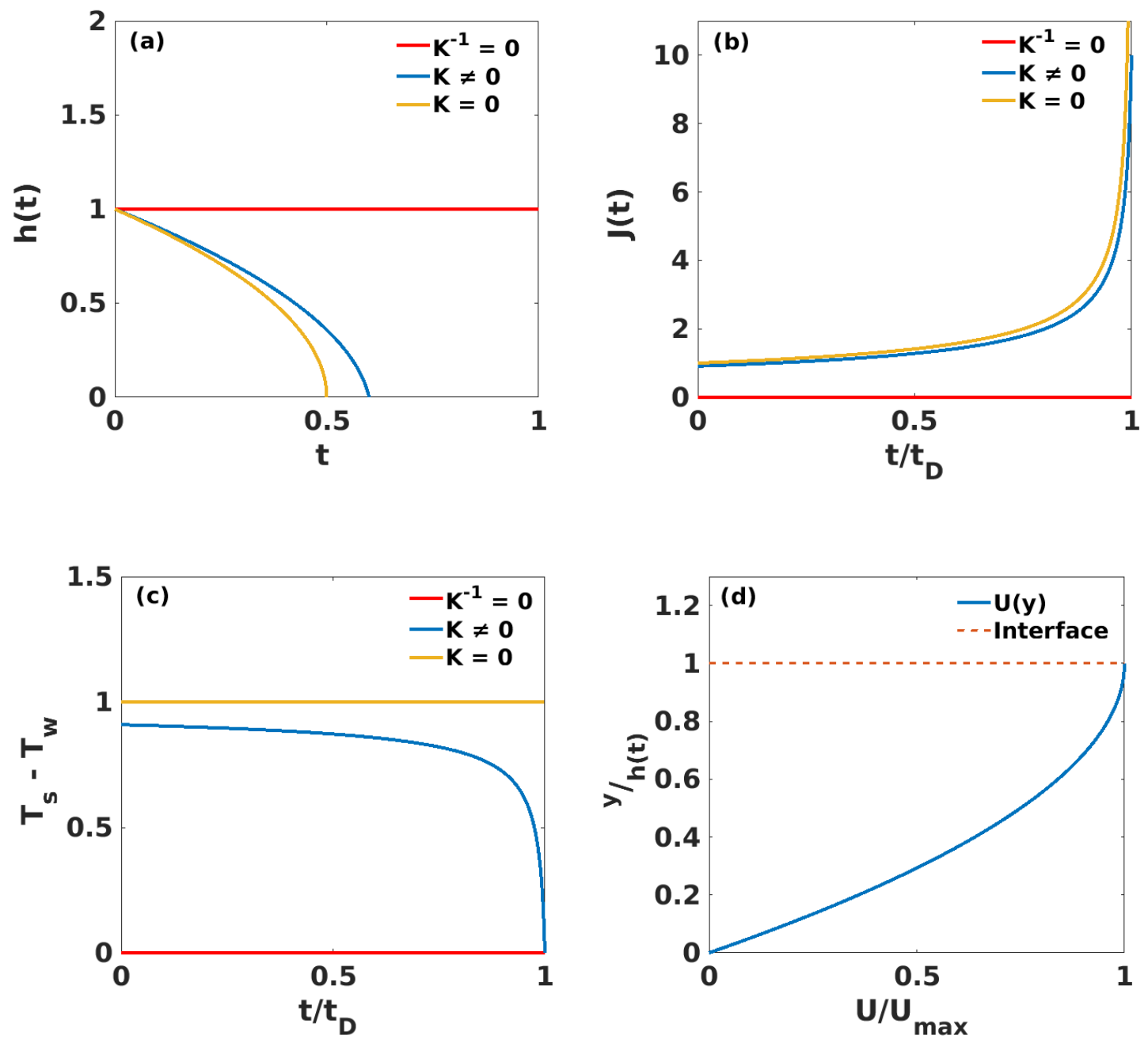

Figure 2.3: (a) Film height, (b) Mass flux across interface, (c) Temperature gradient across the film and (d) horizontal velocity profile, for base state through time. Note that $T_{s}$ is the interface temperature, $T_{w}$ is wall temperature and $t_{D}$ is dry out time. 


\subsection{Long wave theory}

\subsubsection{The Benney equation BE}

Falling liquid films with low flow rates (small $R e$ ) belong to the drag-gravity regime. In this regime, the inertia is weak and the surface tension is strong. Therefore the base state does not change significantly and the slope of interface remains smooth. It is valid then to propose a long wave expansion in terms of the film parameter $\left(\epsilon=\bar{h}_{N} / l\right)$, which is the ratio between the film thickness and the wavelength. This expansion states that the dependent variables $(u, v, T, p)$ change very slowly in space and time as long as the inertia is weak and surface tension is strong. This was done first for isothermal films by Benny [11, where he performed a long-wave expansion of the governing equations and boundary conditions in terms of the film parameter $(\epsilon)$. He also obtained one single equation which describes the evolution of the interface, usually called the Benny equation.

In this monograph, the same procedure done by [30] is followed to obtain the Benny type equation for evaporating falling liquid films. First the time and space are scaled:

$$
\xi=\epsilon x, \quad \zeta=y, \quad \tau=\epsilon t
$$

In order to retain the terms causing hydrodynamic and thermal instabilities, it is assumed that the parameters $(R e, P r, M, C t)$ are of $\mathcal{O}(1)$ [1,36]. with regards to the evaporative instability terms, we assume that $E$ is of $\mathcal{O}(\epsilon)$ and D is of $\mathcal{O}\left(\epsilon^{2}\right)$, in order to retain the effects of mass loss and vapor recoil in the normal stress boundary condition

$$
(E, D)=\left(\epsilon \bar{E}, \epsilon^{2} \bar{D}\right)
$$

where the barred quantities are $\mathcal{O}(1)$. Finally surface tension is assumed of order

$\mathcal{O}\left(\epsilon^{-2}\right)$ corresponding to strong surface tension effects. The dependent variables are expanded ass follows: 


$$
\begin{aligned}
& u=u_{o}+\epsilon u_{1}+\mathcal{O}\left(\epsilon^{2}\right), \\
& v=\epsilon\left(v_{o}+\epsilon v_{1}\right)+\mathcal{O}\left(\epsilon^{3}\right), \\
& T=T_{o}+\epsilon T_{1}+\mathcal{O}\left(\epsilon^{2}\right), \\
& p=p_{o}+\epsilon p_{1}+\mathcal{O}\left(\epsilon^{2}\right), \\
& J=J_{o}+\epsilon J_{1}+\mathcal{O}\left(\epsilon^{2}\right),
\end{aligned}
$$

where, $(u, v, p, T, J)$ are assumed of $\mathcal{O}(1)$ and $\mathrm{v}$ is of order $\mathcal{O}(\epsilon)$ in order to preserve continuity, while $h(x, t)$ is an unknown function of $\mathcal{O}(1)$. The expansions are substituted in the non-dimensional governing equations and boundary conditions 2.13 - 2.15), the velocity, temperature and mass flux solutions are obtained up to $\mathcal{O}(1)$ in terms of the unknown function $h(x, t)$. The velocity in the streamwise direction $(u)$ is obtained as:

$$
\begin{aligned}
& u(\xi, \zeta, \tau)=\left(h \zeta-\frac{1}{2} \zeta^{2}\right)+\epsilon\left\{\left(C_{t} h_{\xi}-\Omega\right)\left(\frac{1}{2} \zeta^{2}-h \zeta\right)\right. \\
& \left.+\frac{3}{2} \operatorname{Reh}_{\tau}\left(\frac{1}{3} \zeta^{3}-h^{2} \zeta\right)+\frac{1}{2} \operatorname{Reh}_{\xi} h\left(\frac{1}{4} \zeta^{4}-h^{3} \zeta\right)+M\left(\frac{h}{h+K}\right)_{\xi}\right\}+O\left(\epsilon^{2}\right),
\end{aligned}
$$

where,

$$
\Omega=V_{r} \frac{h_{\xi}}{(k+h)^{3}}+W e h_{\xi \xi \xi} .
$$

Moreover, the velocity component in $y$-direction at the free surface $\zeta=h$ :

$$
\begin{gathered}
v(\xi, h, \tau)=\epsilon\left\{-\frac{1}{2} h^{2} h_{\xi}\right\}+\epsilon^{2}\left\{C t h^{2}\left(\frac{1}{3} h h_{\xi \xi}+\frac{1}{2} h_{\xi}^{2}\right)-h^{2}\left(\frac{1}{3} h \Theta_{\xi}+\frac{1}{2} h_{\xi} \Theta\right)\right. \\
\left.+3 \operatorname{Reh}^{3}\left(\frac{5}{24} h h_{\tau \xi}+\frac{1}{2} h_{\xi} h_{\tau}\right)+\frac{1}{40} \operatorname{Reh}^{5}\left(39 h_{\xi}^{2}+9 h h_{\xi \xi}\right)-\frac{M}{2}\left(\frac{h}{h+k}\right)_{\xi \xi} h^{2}\right\}+O\left(\epsilon^{3}\right) .
\end{gathered}
$$

The pressure equation:

$$
p=C_{t}(h-\zeta)+\frac{V_{r}}{2} \frac{1}{(h+K)^{2}}-W e h_{\xi \xi}+O(\epsilon),
$$

where the first term is the hydrostatic pressure, the second and the third are vapor recoil and surface tension forces, respectively. The temperature and mass 
flux are obtained as well:

$$
\begin{gathered}
T=1-\frac{\zeta}{h+K}+O(\epsilon), \text { and } \\
J=\frac{1}{h+K}-\epsilon\left\{\frac{P r R e h^{3}}{(K+h)^{3}}\left(h_{\tau}+\frac{1}{8}(39 h+3 K) h_{\xi} h\right)\right\}+O\left(\epsilon^{2}\right) .
\end{gathered}
$$

Substituting the solutions in the kinematic boundary condition $(2.15$,a) yields to the interface evolution equation:

$$
\begin{aligned}
& h_{\tau}+h^{2} h_{\xi}+\frac{\bar{E}}{h+K}+\epsilon\left[\frac{2}{5} \operatorname{Reh}_{\xi} h^{6}+\frac{1}{3} W e h^{3} h_{\xi \xi \xi}-\frac{1}{3} C t h^{3} h_{\xi}\right. \\
& \left.+\frac{K M}{2} \frac{h_{\xi} h^{2}}{(h+K)^{2}}+V_{r} \frac{h^{3} h_{\xi}}{(h+K)^{3}}\right]_{\xi}+\epsilon \frac{5}{2} \operatorname{Re} \bar{E}\left[h^{3} \frac{h^{3} h_{\xi}}{(h+K)}-\frac{1}{4} \bar{E} \frac{h^{4} h_{\xi}}{(h+K)^{2}}\right] \\
& +\epsilon \bar{E} \operatorname{Pr} \operatorname{Re}\left[\frac{7}{40} \frac{h^{5} h_{\xi}}{(h+K)^{3}}+\bar{E} \frac{h^{3}}{(h+K)^{4}}-\frac{3}{8} \frac{h^{4} h_{\xi} h K}{(h+K)^{3}}\right]=0 .
\end{aligned}
$$

The first term represents the film evolution in time, while the second term governs the spacial development of the wave due to mean flow. Inertia, surface tension, and hydrostatic pressure forces are represented by the fourth, fifth, and sixth terms, respectively. The seventh and eighth terms are a result of adding the Marangoni and vapor recoil effects to the Benny equation. Finally, all the terms proportional to $\bar{E}$ represents the mass loss effect, which is responsible for thinning the film until it reaches dry out. The fourth term explains the reason why Benny type equations are only valid when $R e$ is small: it shows that inertia is $\mathcal{O}(\epsilon)$ and therefore it is assumed to be small, however for larger Reynolds numbers, inertia is not negligible, which causing the long wave theory to predict a wrong behavior of the interface evolution.

The interface evolution equation obtained earlier can represent several scenarios. For an isothermal film $\left(M=0, V_{r}=0, E=0\right)$, equation 2.32 is reduced to the evolution equations obtained by many authors in the literature, e.g. see [11,37]. When the Marangoni effect is added $\left(M \neq 0, V_{r}=0, E=0\right)$, the mass flux $J=0$, and the parameter $K^{-1}$ is no longer a measure of evaporation equilibrium and instead it is considered as the Biot number $B$ as mentioned earlier. 
When evaporation effects are included, we recover the equation obtained by [29] but with different scaling parameters. Moreover, if the sign of the hydrostatic pressure term is switched, we recover the evolution equation of an evaporating film falling beneath an inclined surface derived by [38].

The interface evolution equation is a highly non-linear differential equation which can numerically be solved to study the linear and non-linear dynamics of an evaporating falling liquid film. However, in the scope of this monograph, this equation will be linearized with respect to infinitesimal disturbances to study the linear stability of these films in the limit of weak inertia (Reynolds $\mathcal{O}(1)$ ) and longwaves $(k \ll 1)$.

\subsubsection{Film base state}

An exact base state solution is available for the interface evolution equation (2.32), It can be obtained as previously mentioned by setting the spacial derivatives to zero and then obtain the solution as a function of time. $\left(\partial_{\xi}=0 \quad \partial_{\tau} \neq 0\right)$

$$
\begin{gathered}
h_{\tau}+\frac{E}{(h+K)}+\epsilon\left\{\frac{E^{2} P e h^{3}}{(h+K)^{4}}\right\}=0, \\
h_{o}=\Omega-K+\epsilon \frac{E P e}{\Omega}\left\{\frac{K\left(2 K^{2}+6 K+3\right)}{1+K}+\frac{3 K^{2}}{2} \ln \frac{\Omega}{1+K}+\frac{K^{3}}{\Omega}-3 K \Omega-E \tau\right\}+O\left(\epsilon^{2}\right),
\end{gathered}
$$

where, $\Omega=\left((1+K)^{2}-2 \bar{E} \tau\right)^{1 / 2}$. Since the inertia is not taken in consideration in this analysis, the base state (2.34) does not depend on Reynolds number $(R e)$ and mainly depends on the evaporation number $(E)$. For a non-volatile film we recover the film base state found by [7] for isothermal films, and [26] for films with Marangoni effect. 


\section{Chapter 3}

\section{Linear stability analysis: methodologies}

Linear stability analysis is performed by studying the stability of falling films with respect to infinitesimal perturbation (primary instability). Linear stability analysis is a fundamental step in understanding the evolution of these falling liquid films, from a stable state to a chaotic one [1]. In this chapter, the temporal stability of the flow/interface base state is considered, simply, the development of the infinitesimal disturbances is studied in time only. The first part focuses on utilizing the interface evolution equation 2.32 in linear stability analysis. In the next section, 3.2, the well known Orr-Sommerfled eigenvalue problem is extended to include evaporation effects. In Section 3.3, energy balance analysis is carried out for the perturbation in order to to understand the mechanisms of different instabilities, and the way they interact with one another. Finally, a spectral collocation numerical scheme to solve the Orr-Sommerfeld eigenvalue problem is presented along with it is validation, in sections 3.4 and 3.5 respectively. 


\subsection{Long wave theory}

We perform linear stability analysis by adding an infinitesimal perturbation $\tilde{h}(\tau, \xi)$ to the interface

$$
h=h_{o}(\tau)+\tilde{h}(\tau, \xi) .
$$

Then the perturbation is assumed in the form of normal mode:

$$
\tilde{h}(\tau, \xi)=H e^{i(k \xi-\omega \tau)}
$$

where $\omega$ is th complex angular frequency, while $k$ and $H(\tau)$ are real and represent the wavenumber of the perturbation and amplitude, respectively. By substituting the expansion (eq. 3.2) into the evolution equation (eq. 2.32) and linearizing for $\tilde{h}<<1$ the following relation is obtained:

$$
\frac{\dot{H}}{H}=\omega(\tau)-i k c(\tau)+\omega_{e f f}(\tau),
$$

where, $\omega(\tau)$ is the temporal growth rate, $c(\tau)$ is the phase speed, and $\omega_{\text {eff }}$ is the effective growth rate. These terms are defined as follows:

$$
\begin{gathered}
\omega(\tau)=\epsilon k^{2}\left[\frac{2}{5} R e h_{o}^{6}-\frac{1}{3} C t h_{o}^{3}-\frac{1}{3} \bar{W} e h_{o}^{3} k^{2}+\frac{M K h_{o}^{2}}{2\left(h_{o}+K\right)^{2}}+\frac{1}{3} \frac{V_{r} h_{o}^{3}}{\left(h_{o}+K\right)^{3}}\right] \\
c(\tau)=h_{o}^{2}+\epsilon\left[\frac{5 R e \bar{E} h_{o}^{3}}{2\left(h_{o}+K\right)}-\frac{15 R e h_{o}^{4} \bar{E}}{24\left(h_{o}+K\right)^{2}}+\frac{7 \bar{E} P e h_{o}^{5}}{40\left(h_{o}+K\right)^{3}}-\frac{3 \bar{E} P e h_{o}^{4} K}{8\left(h_{o}+K\right)^{3}}\right] \\
\omega_{e f f}(\tau)=\frac{\bar{E}}{\left(h_{o}+K\right)^{2}}+\epsilon \bar{E}^{2} P e \frac{h_{o}^{2}\left(3 K-h_{o}\right)}{\left(h_{o}+K\right)^{5}}
\end{gathered}
$$

If the temporal growth rate is positive $\omega(\tau)>0$, the disturbance grows exponentially in time and the base state is unstable. On the other hand, if $\omega(\tau)<0$ the base state is stable and the disturbance is damped. Furthermore, if $\omega(\tau)=0$, the disturbance is neither amplified or damped, but instead the film base state solution is shifted and travels with a constant phase speed $c(\tau)$. The sign of the different terms in equation (3.4) indicates the role every term plays, the first term shows the destabilizing effect of the mean flow (positive), while the second and third terms shows the stabilizing effects of the hydrostatic pressure and surface tension respectively (negative). Marangoni and vapor recoil effects are destabilizing by their nature, and therefore they appear with a positive sign in the last 
two terms. Finally, the term $\left(\omega_{e f f}\right)$ seems to contribute positively to the growth rate in equation (3.3), however it does not affect the exponential growth of the disturbance, and it only indicates that initial disturbance amplitude grows as the film thins 29,30$]$.

Moreover, by setting $\omega(\tau)=0$ and rearranging equation 3.4 , the critical condition is easily obtained:

$$
0=\frac{2}{5} R e h_{o}^{6}-\frac{1}{3} C t h_{o}^{3}+\frac{M K h_{o}^{2}}{2\left(h_{o}+K\right)^{2}}+\frac{1}{3} \frac{V_{r} h_{o}^{3}}{\left(h_{o}+K\right)^{3}} .
$$

When $M=0, V_{r}=0, E=0$, the above relation reduces to the critical condition of surface wave instability (H-mode) obtained in [8]. When vapor recoil effect is included, equation (3.7) has two solutions for $R e$, one corresponds the vapor recoil instability (E-mode), while the other one corresponds to the surface wave instability (H-mode) modified by evaporation. The same applies when Marangoni effect is included.

In summary, the primary instability of evaporating falling liquid films is resolved under two strict assumptions, first the inertia is weak (low Re), and second, the disturbance wavenumber is small.

\subsection{Orr-Sommerfeld eigenvalue problem}

The Orr-Sommerfeld (OS) eigenvalue problem is the classical tool used to study the linear stability of film flows. It has no restrictions on inertia nor disturbance wavenumber, and therefore it gives a wider insight into the linear stability analysis. The OS problem was first derived and solved by Goussis and Kelly for heated falling films [39]. Afterwards, it was extensively used in the literature to study linear stability of isothermal and heated falling films with different effects, for example see [40]. However, for evaporating films, the OS has not been derived to our extent of knowledge. The main originality of this work is to derive the OS problem for evaporating falling liquid films. 
The linear stability of the base state with respect to infinitesimal perturbations is considered by substituting the following in the governing equations and boundary conditions $2.13-2.15$,

$$
\mathbf{v}=(\bar{U}+\tilde{u}, \tilde{v}, \tilde{w}), \quad T=\bar{\Theta}+\tilde{T}, \quad p=\bar{P}+\tilde{p}, \quad h=\bar{h}+\tilde{h}, \quad J=\bar{J}+\tilde{J},
$$

in which the "tilde" quantities are the perturbations. The linearized perturbation equations are obtained by setting $(\tilde{u}, \tilde{v}, \tilde{T}, \tilde{p}, \tilde{h}, \tilde{J} \ll 1)$ :

$$
\begin{aligned}
& \partial_{x} \tilde{u}+\partial_{y} \tilde{v}+\partial_{z} \tilde{w}=0, \\
& 3 \operatorname{Re}\left(\partial_{t} \tilde{u}+\bar{U} \partial_{x} \tilde{u}+D \bar{U} \tilde{v}\right)+\partial_{x} \tilde{p}-\nabla^{2} \tilde{u}=0, \\
& 3 \operatorname{Re}\left(\partial_{t} \tilde{v}+\bar{U} \partial_{x} \tilde{v}\right)+\partial_{y} \tilde{p}-\nabla^{2} \tilde{v}=0, \\
& 3 \operatorname{Re}\left(\partial_{t} \tilde{w}+\bar{U} \partial_{x} \tilde{w}\right)+\partial_{y} \tilde{p}-\nabla^{2} \tilde{w}=0, \\
& 3 \operatorname{Re} \operatorname{Pr}\left(\partial_{t} \tilde{T}+\bar{U} \partial_{x} \tilde{T}+D \bar{\Theta} \tilde{v}\right)-\nabla^{2} \tilde{T}=0,
\end{aligned}
$$

along with the boundary conditions, at the plate $y=0$,

$$
\begin{aligned}
& \tilde{u}=\tilde{v}=\tilde{w}=0, \\
& \tilde{T}=0,
\end{aligned}
$$

and at the free surface $y=h(x, z, t)$

$$
\begin{aligned}
& \tilde{v}=\partial_{t} \tilde{h}+\bar{U} \partial_{x} \tilde{h}+E \tilde{J}, \\
& \tilde{p}=2 \frac{\partial \tilde{v}}{\partial y}+C t \tilde{h}-(W e-M \bar{\Theta}(h)) \nabla_{x z}^{2} \tilde{h}+\frac{V_{r}}{2} \bar{J} \tilde{J}, \\
& \tilde{h}=\partial_{y} \tilde{u}+\partial_{x} \tilde{v}+M\left(\partial_{x} \tilde{T}+\partial_{x} \tilde{h} D \bar{\Theta}\right), \\
& \partial_{y} \tilde{T}=-\tilde{J}+\frac{3 R e V_{r} \bar{J}}{D \mathcal{L}} \tilde{J}, \\
& \tilde{J}=\frac{\tilde{T}}{K}+\frac{D \bar{\Theta}}{K} \tilde{h} .
\end{aligned}
$$

With regards to the interface boundary conditions, the introduction of evaporation effects causes changes to the kinematic $(3.10 a)$, normal stress $(3.10 \mathrm{p})$, and energy $(3.10)$ ) boundary conditions. The kinematic boundary condition gains an additional term proportional to $E$ which accounts for mass loss, as explained in the previous section this term does not contribute to the exponential instability and therefore will be disregarded. Furthermore, the normal stress boundary condition also gains an additional term proportional to $V_{r}$ which represents the vapor 
recoil effect. The first term in the energy boundary condition represent the perturbation in the mass flux across the interface, while the second term represent the perturbation in the kinetic energy imparted to the vapor particles. The later is small compared to the former and therefore will be disregarded [29]. Taylor series expansion have been utilized to obtained the interface boundary conditions at $y=h=\bar{h}+\tilde{h}$, for example the velocity is expanded as $\left.u\right|_{h}=U(\bar{h})+\left.\tilde{u}\right|_{\bar{h}}+D U(\bar{h}) \tilde{h}$, where $D$ is the derivative in $y$-direction.

The following steps are performed to let appear only the perturbations of the normal velocity $(\tilde{v})$, the temperature $(\tilde{T})$ and the film thickness $(\tilde{h})[1$. First the divergence of the linearized Navier-Stokes equations is taken in vector form $\left[\partial_{x}(3.8 . b)+\partial_{y}(3.8 . c)+\partial_{z}(3.8 . d)\right]$, and with the aid of the continuity equation (3.8a), it is obtained

$$
\nabla^{2} \tilde{p}=-6 \operatorname{Re} D \bar{U} \partial_{x} \tilde{v}
$$

Then the two-dimensional Laplacian operator is applied on the linearized ymomentum equation (3.8c), while using (3.11) to eliminate the pressure:

$$
\nabla^{2}\left(3 R e \partial_{t} \tilde{v}-\nabla^{2} \tilde{v}\right)+3 R e\left(1+\bar{U} \nabla^{2}\right) \partial_{x} \tilde{v}=-6 \operatorname{Re} D \bar{U} \partial_{x} \tilde{v}
$$

differentiating the y-momentum equation 2.13 ) with respect to $\mathrm{y}$,

$$
\partial_{y y} \tilde{p}=-3 R e\left(\partial_{y t} \tilde{v}+D \bar{U} \partial_{x} \tilde{v}+\bar{U} \partial_{x y} \tilde{v}\right)+\partial_{y} \tilde{v}^{2}
$$

Next, 3.13 is evaluated at the undeformed interface $h_{o}$ and using the property $\left.\nabla^{2} \tilde{p}\right|_{h}=\left.\nabla_{x z}^{2} \tilde{p}\right|_{h}+\left.\partial_{y y} \tilde{p}\right|_{h}=0$

$$
\nabla_{x z}^{2} \tilde{p}=-3 \operatorname{Re}\left(\partial_{y t} \tilde{v}+U \partial_{x y} \tilde{v}\right)+\partial_{y} \tilde{v}^{2}
$$

from $\nabla_{x z}^{2}$ in $(3.10 \mathrm{~b})$, we get:

$C t \nabla_{x z}^{2} \tilde{h}-(W e-M \bar{\Theta}) \nabla_{x z}^{2} \nabla_{x z}^{2} \tilde{h}+3 \nabla_{x z}^{2} \partial_{y} \tilde{v}+\partial_{y y y} \tilde{v}-3 R e\left(\partial_{y t} \tilde{v}+\bar{U} \partial_{x y} \tilde{v}\right)+V_{r} \bar{J} \tilde{J}$.

Finally, by taking the divergence of the tangential stress boundary conditions $\left[\partial_{x}(3.10 . c)+\partial_{y}(3.10, d)\right]$ and using the continuity equation $\left.3.8 \mathrm{a}\right)$,

$$
\partial_{x} \tilde{h}-M\left(D \bar{\Theta} \nabla_{x z}^{2} \tilde{h}+\nabla_{x z}^{2} \tilde{T}\right)-\left(\nabla_{x z}^{2}-\partial_{y y}\right) \tilde{v}=0
$$


The solution is then assumed in the form of normal modes:

$$
\left\{\begin{array}{c}
\tilde{v} \\
\tilde{T} \\
\tilde{h} \\
\tilde{J}
\end{array}\right\}=\left\{\begin{array}{c}
i k \varphi(y) \\
\tau(y) \\
\eta \\
\mathcal{J}
\end{array}\right\} e^{i(\mathbf{k} \cdot \mathbf{x}-\omega t)}
$$

where, the left hand side shows the perturbation quantities. $\mathbf{x}=(x, z)$, and $\mathbf{k}=\left(k_{x}, k_{z}\right)$ is the complex wavenumber, while $\omega$ is the complex angular frequency. The normal modes solution is then substituted in the linearized governing equations (3.12), (3.8p), and the boundary conditions (3.9), 3.10a), 3.10e), (3.15) and (3.16) leading to the generalized Orr-Sommerfeld eigenvalue problem for evaporating falling films:

$$
\begin{aligned}
& \left(D^{2}-k^{2}\right)^{2} \phi+3 i \operatorname{Re}\left[(\omega-k \bar{U})\left(D^{2}-k^{2}\right)+D^{2} \bar{U} k\right] \phi=0 \\
& \left(D^{2}-k^{2}\right) \tau+3 \operatorname{Pe}[D \Theta \phi-i(\omega-k \bar{U}) \tau]=0
\end{aligned}
$$

along with the wall boundary conditions $(y=0)$,

$$
\begin{aligned}
& \phi(0)=D \phi(0)=0, \\
& \tau(0)=0,
\end{aligned}
$$

and interface boundary conditions $y=h(t)$,

$$
\begin{aligned}
& \phi(h)+i \frac{1}{2} \eta(2 \omega-2 \bar{U} k)=0, \\
& {\left[\left(D^{2}-3 k^{2}\right)+3 i R e(\omega-\bar{U} k)\right] D \phi(h)} \\
& \quad=\eta k^{2}\left[C t+(W e-M \bar{\Theta}) k^{2}\right]+k^{2} V_{r} \bar{J} \mathcal{J}, \\
& \left(D^{2}+k^{2}\right) \phi(h)+M[\eta D \bar{\Theta}+\tau(h)] k^{2}+i k \eta=0, \\
& D \tau(h)+\mathcal{J}=0, \\
& \mathcal{J}=\frac{\tau(h)}{K}+\frac{D \bar{\Theta}}{K} \eta,
\end{aligned}
$$

where the base state quantities (with a bar) in the interface boundary conditions are evaluated at the unreformed interface. 
The way $\mathbf{k}$ and $\omega$ are chosen depends on the type of analysis required.

- For temporal stability analysis, where the evolution of disturbance in time is concerned. The disturbance wavenumber $\mathbf{k}$ is set to be real, then the problem is solved for the complex eigenvalue $\omega$. The imaginary part of $\omega$ is called the temporal growth rate $\omega_{i}$ (subscript $\mathrm{i}$ is used to denote imaginary, while subscript $\mathrm{r}$ denotes real), while $c_{r}=k / \omega_{r}$ is the phase velocity, and $\mathrm{k}=\sqrt{k_{x}^{2}+k_{z}^{2}}$ is the modulus of the wavenumber vector.

- One the other hand, when the evolution of a disturbance in space is the main focus (spatial stability analysis), $\omega$ is assumed to be real and the solution is found for the complex eigenvalue $\mathbf{k}$. The imaginary part of $\mathrm{k}$ is called in this case the spatial growth rate $k_{i}$. For both cases, if the growth rate is positive $\left(\omega_{i} / k_{i}>0\right)$ the disturbance grows in time/space, while if $\left(\omega_{i} / k_{i}<0\right)$ the disturbance is damped.

- Another scenario exists when the evolution of the disturbance in time and space at the same time is targeted (spatial/temporal stability analysis), both $\mathbf{k}$ and $\omega$ are assumed to be complex in this case.

- Moreover, the general Orr-Sommerfeld eigenvalue problem $3.17-3.19$ has two limiting cases, streamwise perturbations $\left(k_{x} \neq 0, k_{z}=0\right)$, and transverse perturbations $\left(k_{x}=0, k_{z} \neq 0\right)$.

In this monograph our focus only goes to temporal stability analysis in the streamwise direction.

\subsubsection{Streamwise perturbations $\left(k_{x}=k, k_{z}=0\right)$}

Isothermal falling liquid films are the most unstable for two dimensional perturbations, and more specifically, for streamwise perturbations [41], this also applies when Marangoni effect is taken into account [39]. The same assumption is used for studying the linear stability of evaporating falling liquid films in this monograph. 
Therefore, the streamfunction is utilized to rewrite the perturbation velocity amplitude as follows:

$$
\phi(y)=-i k_{x} \varphi(y)
$$

by applying the previous transformation and setting $\left(k_{x}=k, \omega=k c\right)$ in 3.17 3.19), the streamwise OS eigenvalue problem is obtained as follows:

$$
\begin{aligned}
& \left(D^{2}-k^{2}\right)^{2} \varphi+3 \operatorname{Reik}\left[(c-\bar{U})\left(D^{2}-k^{2}\right)+D^{2} \bar{U}\right] \varphi=0, \\
& \left(D^{2}-k^{2}\right) \tau+3 \operatorname{RePrik}[D \Theta \varphi+(c-\bar{U}) \tau]=0,
\end{aligned}
$$

with boundary conditions at the wall

$$
\begin{aligned}
& \varphi(0)=D \varphi(0)=0, \\
& \tau(0)=0,
\end{aligned}
$$

and at the free surface

$$
\begin{aligned}
& \eta=\frac{\varphi(h)}{(c-\bar{U})}, \\
& {\left[\left(D^{2}-3 k^{2}\right)+3 \operatorname{Reik}(c-\bar{U})\right] D \varphi(h)} \\
& -i \eta k\left[C t+(W e-M \bar{\Theta}) k^{2}\right]-i k V_{r} \bar{J} \mathcal{J}=0, \\
& \left(D^{2}+k^{2}\right) \varphi(h)+i k M[\eta D \bar{\Theta}+\tau(h)]-\eta=0, \\
& D \tau(h)+\frac{\tau(h)}{K}-\frac{\bar{J}}{K} \eta=0, \\
& \mathcal{J}=\frac{\tau(h)}{K}+\frac{D \bar{\Theta}}{K} \eta \text {. }
\end{aligned}
$$

The OS eigenvalue problem in $3.20-3.22)$ outperforms the long wave theory presented earlier when linear stability analysis is performed. Not only the fact that OS eigenvalue problem is valid for wider range of $R e$ and $k$ parameters, but it also provides a solution of the perturbation quantities $(\tilde{u}, \tilde{v}, \tilde{T}, \tilde{J}, \eta)$. These quantities are necessary to determine the energy balance of the perturbation, which gives better insight on how physical effects can cause or demolish different instability modes. We elaborate more on this in the next section. 


\subsection{Perturbation energy analysis}

There are several forces presented in evaporating falling liquid films, for example, surface tension, hydrostatic pressure, and vapor recoil. In order to understand how these forces could enhance/oppose the perturbation growth rate for different parameters such as $(k, R e, \beta)$, it is necessary to analyze the rate of change of the perturbation kinetic energy . This can be achieved by deriving the "kinetic energy balance". This was done first for isothermal films, where it was found that the work done by the perturbation shear stress at the interface is the main contributor to the energy of the perturbation [9]. The "kinetic energy balance" was then extended to include Marangoni effect for heated falling films. The contribution of thermocapillary forces to the perturbation kinetic energy was analyzed for different values of Reynolds number $(R e)$ and wavenumber $(k)$ [39]. Here, the same procedure is followed in order to derive the "kinetic energy balance" including vapor recoil effect $\left(V_{r}\right)$.

The kinetic energy balance of the perturbation can be obtained by multiplying the perturbation $x$-momentum equation $(3.8 \mathrm{~b}$ ) by $\tilde{u}$ and the perturbation $y$ momentum equation (3.8.c) by $\tilde{v}$ and summing them up together,

$$
\begin{aligned}
\frac{1}{2}\left(\partial_{t}+U \partial_{x}\right)\left(\tilde{u}^{2}+\tilde{v}^{2}\right)= & -D U \tilde{u} \tilde{v}-\frac{1}{R e}\left(\tilde{u} \partial_{x}+\tilde{v} \partial_{y}\right) \tilde{p} \\
& +\frac{1}{3 R e}\left[\tilde{u}^{2}\left(\partial_{x x}+\partial_{y y}\right)+\tilde{v}^{2}\left(\partial_{x x}+\partial_{y y}\right)\right] .
\end{aligned}
$$

Since the perturbation can be decomposed into a sum of periodic functions through Fourier transform, and based on the Parseval theorem, it is valid to assume that the kinetic energy of the perturbation is the sum of all the kinetic energies of the periodic functions . Therefore the kinetic energy balance is derived for one periodic function with wavelength $(\lambda=2 \pi / k)$. By integrating (3.23) over the domain, and dividing by $\lambda$ for averaging:

$$
\begin{aligned}
\frac{1}{2 \lambda} \int_{0}^{h} & \int_{0}^{\lambda}\left(\partial_{t}+U \partial_{x}\right)\left(\tilde{u}^{2}+\tilde{v}^{2}\right) d x d y \\
& =-\frac{1}{\lambda} \int_{0}^{h} \int_{0}^{\lambda} D U \tilde{u} \tilde{v} d x d y-\frac{1}{\operatorname{Re\lambda }} \int_{0}^{h} \int_{0}^{\lambda}\left(\tilde{u} \partial_{x}+\tilde{v} \partial_{y}\right) \tilde{p} d x d y \\
& +\frac{1}{3 \operatorname{Re\lambda }} \int_{0}^{h} \int_{0}^{\lambda}\left[\tilde{u}^{2}\left(\partial_{x x}+\partial_{y y}\right)+\tilde{v}^{2}\left(\partial_{x x}+\partial_{y y}\right)\right] d x d y .
\end{aligned}
$$


- The first term is simplified as follows:

$$
\begin{aligned}
\int_{0}^{h} & \int_{0}^{\lambda} \frac{1}{2}\left(\partial_{t}+U \partial_{x}\right)\left(\tilde{u}^{2}+\tilde{v}^{2}\right) d x d y \\
& =\frac{1}{2} \frac{d}{d t} \int_{0}^{h} \int_{0}^{\lambda}\left(\tilde{u}^{2}+\tilde{v}^{2}\right) d x d y+\frac{1}{2} \int_{0}^{h} U(y) \int_{0}^{\lambda} \partial_{x}\left(\tilde{u}^{2}+\tilde{v}^{2}\right) d x d y \\
& =\frac{1}{2} \frac{d}{d t} \int_{0}^{h} \int_{0}^{\lambda}\left(\tilde{u}^{2}+\tilde{v}^{2}\right) d x d y+\frac{1}{2} \int_{0}^{h} U(y)\left[\left(\tilde{u}^{2}+\tilde{v}^{2}\right)\right]_{0}^{\lambda} d y \\
& =\frac{1}{2} \frac{d}{d t} \int_{0}^{h} \int_{0}^{\lambda}\left(\tilde{u}^{2}+\tilde{v}^{2}\right) d x d y .
\end{aligned}
$$

- The third term is first simplified by integrating by parts, and then using the continuity equation (3.8.a):

$$
\int_{0}^{h} \int_{0}^{\lambda}\left(\tilde{u} \partial_{x}+\tilde{v} \partial_{y}\right) \tilde{p} d x d y=-\int_{0}^{h}[\tilde{u} \tilde{p}]_{0}^{\lambda} d y-\int_{0}^{\lambda}[\tilde{v} \tilde{p}]_{0}^{h} d x=-\left.\left.\int_{0}^{\lambda} \tilde{v}\right|_{h} \tilde{p}\right|_{h} d x .
$$

Then by substituting for $\left.\tilde{p}\right|_{h}$ using the normal stress boundary condition $(3.10 . \mathrm{b})$,

$$
\begin{aligned}
& -\left.\left.\int_{0}^{\lambda} \tilde{v}\right|_{h} \tilde{p}\right|_{h} d x= \\
& \quad-\left.\int_{0}^{\lambda} \tilde{v}\right|_{h}\left(C t \tilde{h}-W e \partial_{x x} \tilde{h}+M \bar{\Theta}(h) \partial_{x x} \tilde{h}+2 \partial_{y} \tilde{v}+V_{r} \bar{J} \tilde{J}\right) d x .
\end{aligned}
$$

By using the continuity equation (3.8.a) again and integration by parts we also can write:

$$
\begin{aligned}
\left.\left.\int_{0}^{\lambda} \tilde{v}\right|_{h} \partial_{y} \tilde{v}\right|_{h} d x & =-\left.\left.\int_{0}^{\lambda} \tilde{v}\right|_{h} \partial_{x} \tilde{u}\right|_{h} d x \\
& =-\left[\left.\left.\tilde{u}\right|_{h} \tilde{v}\right|_{h}\right]_{0}^{\lambda}+\left.\left.\int_{0}^{\lambda} \tilde{v}\right|_{h} \tilde{u}\right|_{h} d x \\
& =\left.\left.\int_{0}^{\lambda} \tilde{v}\right|_{h} \tilde{u}\right|_{h} d x,
\end{aligned}
$$

after using the previous relation, the third term is written as:

$$
\begin{aligned}
\int_{0}^{h} \int_{0}^{\lambda}\left(\tilde{u} \partial_{x}+\tilde{v} \partial_{y}\right) \tilde{p} d x d y= & \left.\int_{0}^{\lambda} \tilde{v}\right|_{h}\left(W e \partial_{x x} \tilde{h}-M \bar{\Theta}(h) \partial_{x x} \tilde{h}-V_{r} \bar{J} \tilde{J}-C t \tilde{h}\right) d x \\
& +\left.\int_{0}^{\lambda} \tilde{u}\right|_{h}\left(\left.\partial_{y} \tilde{u}\right|_{h}-\left.\partial_{x} \tilde{v}\right|_{h}\right) d x .
\end{aligned}
$$


- The fourth term in equation $(3.24)$ is expanded as follows:

$$
\begin{aligned}
\int_{0}^{h} \int_{0}^{\lambda}\left[\tilde{u}^{2}\left(\partial_{x x}+\partial_{y y}\right)+\tilde{v}^{2}\left(\partial_{x x}+\partial_{y y}\right)\right] d x d y \\
=\int_{0}^{h}\left[\tilde{u} \partial_{x} \tilde{u}+\tilde{v} \partial_{x} \tilde{v}\right]_{0}^{\lambda} d y+\int_{0}^{\lambda}\left[\tilde{u} \partial_{y} \tilde{u}+\tilde{v} \partial_{y} \tilde{v}\right]_{0}^{h} d y \\
\quad-\int_{0}^{h} \int_{0}^{\lambda}\left[\left(\partial_{x} \tilde{u}\right)^{2}+\left(\partial_{y} \tilde{u}\right)^{2}+\left(\partial_{x} \tilde{v}\right)^{2}+\left(\partial_{y} \tilde{v}\right)^{2}\right] \\
=\int_{0}^{\lambda}\left[\tilde{u} \partial_{y} \tilde{u}+\tilde{v} \partial_{y} \tilde{v}\right]_{0}^{h} d y-\int_{0}^{h} \int_{0}^{\lambda}\left[\left(\partial_{x} \tilde{u}\right)^{2}+\left(\partial_{y} \tilde{u}\right)^{2}+\left(\partial_{x} \tilde{v}\right)^{2}+\left(\partial_{y} \tilde{v}\right)^{2}\right] d x d y .
\end{aligned}
$$

Then equation 3.24 is rewritten again with the simplified terms:

$$
\begin{aligned}
\frac{1}{2 \lambda} \frac{d}{d t} \int_{0}^{h} \int_{0}^{\lambda}\left(\tilde{u}^{2}+\tilde{v}^{2}\right) d x d y \\
=-\frac{1}{\lambda} \int_{0}^{h} \int_{0}^{\lambda} D U \tilde{u} \tilde{v} d x d y+\left.\frac{1}{3 \operatorname{Re} \lambda} \int_{0}^{\lambda} \tilde{u}\right|_{h}\left(\left.\partial_{y} \tilde{u}\right|_{h}-\left.\partial_{x} \tilde{v}\right|_{h}\right) d x \\
\quad+\left.\frac{1}{3 \operatorname{Re} \lambda} \int_{0}^{\lambda} \tilde{v}\right|_{h}\left(W e \partial_{x x} \tilde{h}-M \Theta(h) \partial_{x x} \tilde{h}-V_{r} \bar{J} \tilde{J}-C t \tilde{h}\right) d x \\
\quad-\frac{1}{3 \operatorname{Re} \lambda} \int_{0}^{h} \int_{0}^{\lambda}\left[2\left(\partial_{x} \tilde{u}\right)^{2}+\left(\partial_{y} \tilde{u}\right)^{2}+\left(\partial_{x} \tilde{v}\right)^{2}\right] d x d y
\end{aligned}
$$

Finally by using the continuity equation and integration by parts:

$$
\begin{aligned}
0 & =\int_{0}^{h} \int_{0}^{\lambda}\left(\partial_{x} \tilde{u}+\partial_{y} \tilde{v}\right)^{2} d x d y \\
& =\int_{0}^{h} \int_{0}^{\lambda}\left[\left(\partial_{x} \tilde{u}\right)^{2}+\left(\partial_{y} \tilde{v}\right)^{2}\right]+2 \int_{0}^{h} \int_{0}^{\lambda}\left(\partial_{x} \tilde{u} \partial_{y} \tilde{v}\right) d x d y \\
& =\int_{0}^{h} \int_{0}^{\lambda}\left[\left(\partial_{x} \tilde{u}\right)^{2}+\left(\partial_{y} \tilde{v}\right)^{2}\right]+2 \int_{0}^{h}\left[\tilde{u} \partial_{y} \tilde{v}\right]_{0}^{\lambda} d y \\
& -2 \int_{0}^{\lambda}\left[\tilde{u} \partial_{x} \tilde{v}\right]_{0}^{h} d x+2 \int_{0}^{h} \int_{0}^{\lambda} \partial_{y} \tilde{u} \partial_{x} \tilde{v} d x d y \\
= & \int_{0}^{h} \int_{0}^{\lambda}\left[\left(\partial_{x} \tilde{u}\right)^{2}+\left(\partial_{y} \tilde{v}\right)^{2}+\partial_{y} \tilde{u} \partial_{x} \tilde{v}\right] d x d y-\left.\left.2 \int_{0}^{\lambda} \tilde{u}\right|_{h} \partial_{x} \tilde{y}\right|_{h} d x .
\end{aligned}
$$

The final form of the kinetic energy balance is:

$$
K I N+S T E+H Y D=R E Y+S H E+D I S+M A R+V R E
$$


where the different energy terms are defined as follows:

$$
\begin{aligned}
K I N & =\frac{1}{2 \lambda} \frac{d}{d t} \int_{0}^{\lambda} \int_{0}^{h}\left(\tilde{u}^{2}+\tilde{v}^{2}\right) d y d x \\
S T E & =-\frac{W e}{3 \operatorname{Re} \lambda} \int_{0}^{\lambda}\left[\left.\tilde{v}\right|_{h}\left(\partial_{x x} \tilde{h}\right)\right] d x \\
H Y D & =\left.\frac{C t}{3 \operatorname{Re} \lambda} \int_{0}^{\lambda} \tilde{v}\right|_{h}(\tilde{h}) d x \\
S H E & =-\left.\frac{1}{3 \operatorname{Re} \lambda} D^{2} U \int_{0}^{\lambda} \tilde{u}\right|_{h} \tilde{h} d x \\
R E Y & =-\frac{1}{\lambda} \int_{0}^{\lambda} \int_{0}^{h} \tilde{u} \tilde{v} D U d y d x \\
D I S & =-\frac{1}{3 \operatorname{Re} \lambda} \int_{0}^{\lambda} \int_{0}^{h}\left[2\left(\partial_{x} \tilde{u}\right)^{2}+\left(\partial_{y} \tilde{u}+\partial_{x} \tilde{v}\right)^{2}+2\left(\partial_{y} \tilde{v}\right)^{2}\right] d y d y \\
M A R & =-\frac{M}{3 \operatorname{Re} \lambda} \int_{0}^{\lambda}\left[\left.\Theta(h) \tilde{v}\right|_{h} \partial_{x x} \tilde{h}+\left.\tilde{u}\right|_{h}\left(D \Theta(h) \tilde{h}+\partial_{x} \tilde{T}\right)\right] d x \\
V R E & =-\left.\frac{J V_{r}}{3 \operatorname{Re} \lambda} \int_{0}^{\lambda} \tilde{v}\right|_{h} \tilde{J} d x .
\end{aligned}
$$

The Vapor recoil energy (VRE) can be rewritten using the linearized constitutive equation (3.10) as:

$$
V R E=-\left.\frac{\bar{J} V_{r}}{3 \operatorname{Re} \lambda K} \int_{0}^{\lambda} \tilde{v}\right|_{h}\left[\left.\tilde{T}\right|_{h}+\left.D \bar{\Theta}\right|_{h} \tilde{h}\right] d x .
$$

The real part of the perturbation quantities $(\tilde{u}, \tilde{v}, \tilde{T}, \eta)$ in equation 3.33$)$ is defined as follows: (See Appendix B for derivation details )

$$
\begin{aligned}
& \tilde{u}=\left[D \varphi_{r} \cos (\theta)-D \varphi_{i} \sin (\theta)\right] e^{k c_{i} t} \\
& \tilde{v}=\left[\varphi_{i} \cos (\theta)+\varphi_{i} \sin (\theta)\right] k e^{k c_{i} t} \\
& \tilde{T}=\left[\tau_{r} \cos (\theta)+\tau_{i} \sin (\theta)\right] e^{k c_{i} t} \\
& \tilde{h}=\left[\eta_{r} \cos (\theta)+\eta_{i} \sin (\theta)\right] e^{k c_{i} t}
\end{aligned}
$$

where, $\theta=k\left(x-c_{r} t\right)$, while, $\eta_{r}$ and $\eta_{i}$ are:

$$
\eta_{r}=\frac{\varphi_{r}(h)\left(c_{r}-\bar{U}(h)\right)+\varphi_{i}(h) c_{i}}{\left(c_{r}-\bar{U}(h)\right)^{2}+c_{i}^{2}}, \quad \eta_{i}=\frac{\varphi_{i}(h)\left(c_{r}-\bar{U}(h)\right)-\varphi_{r}(h) c_{i}}{\left(c_{r}-\bar{U}(h)\right)^{2}+c_{i}^{2}}
$$


The three terms on the right hand side represent the way the total energy is distributed to the perturbation.

- KIN represents the rate of change of the perturbation kinetic energy and it is proportional to the growth rate $\left(\omega_{i}\right)$. If the flow is unstable $\left(\omega_{i}>0\right)$, the kinetic energy of the perturbation is increasing and KIN is positive. Moreover, KIN is negative if the flow is stable $\left(\omega_{i}<0\right)$, and zero if the flow is neutrally stable $\left(\omega_{i}=0\right)$.

- HYD represents the rate of work done against the hydrostatic pressure. It is also proportional to the growth rate, it indicates that the energy is released from the perturbation if the growth rate is negative $\left(\omega_{i}<0\right)$, , however for an unstable flow $\left(\omega_{i}>0\right)$, HYD is positive, which means that energy is given to the perturbation in order to overcome the stabilizing effect of hydrostatic pressure.

- STE represents the work done against the stabilizing effect of surface tension. It is proportional to the growth rate and behaves the same way as HYD. It indicates that the energy it released or given to the perturbation in order to overcome the stabilizing forces of surface tension depending on whether the flow is stable or unstable, respectively. For disturbances with large wavenumber $(k)$, surface tension is the main restoring force and hydrostatic pressure is negligible in comparison. For disturbances with small wavenumber, the opposite applies.

With regards to the terms on the right hand side, they represent the rate of change of available energy. SHE represents the work done by perturbation shear stress and always positive. MAN and VRE have destabilizing nature, they show the rate of energy transfer to the perturbation by Marangoni and vapor recoil forces, respectively. REY is the work done by Reynolds stress and is negligible in the range of Reynolds numbers used in this monograph. Finally DIS is the rate of energy dissipation by viscosity and it is always negative. Consequently, the total energy available for the perturbation is the net sum of DIS, SHE, MAN, and VRE,. If the sum is positive, the flow is unstable and the disturbance grows, otherwise the flow is stable and the disturbance is damped. 
The evaluation of the energy terms in (3.33) requires the solution of the OS eigenvalue problem consisting of the eigenvalue $\omega$ and the eigenfunctions $\varphi(y)$ and $\tau(y)$, the next section suggests a numerical scheme that provides accurate results of these quantities.

\subsection{Chebyshev spectral method}

Solving the Orr-Sommerfeld problem is not a simple task. Several numerical methods have been developed to obtain an accurate solution of the eigenvalue problem for different hydrodynamic instability problems. It was first solved by Anshus [42] in 1966 for an isothermal falling liquid film. Other methods were developed after, for example, the shooting method [43], and finite difference method [44]. The mentioned methods accurately solved the OS problem, however large storage and computational effort were required. One alternative method is the spectral collocation method, where the domain is discretized using Chebyshev polynomials, it was first developed by [45], and subsequently was used extensively in the literature for its accuracy and simplicity.

First, the Chebyshev spectral method is briefly introduced. The fist kind Chebyshev polynomials can be written in many ways, one is using the trigonometric functions,

$$
T_{n}(z)=\cos \left(n \cos ^{-1}(z)\right),
$$

where $-1<z<1$, and $n$ is the order of the polynomial, another form of presentation is the direct formula,

$$
T_{n}(z)=\frac{1}{2}\left[\left(z+\sqrt{z^{2}-1}\right)^{n}+\left(z-\sqrt{z^{2}-1}\right)^{n}\right] .
$$

The dependent variable is approximated by the Chebyshev expansion

$$
f(z)=\sum_{n=0}^{N} a_{n} T_{n}(z),
$$

where the Chebyshev polynomials are evaluated at the chebyshev points

$$
z_{j}=\cos \left(\frac{j \pi}{N}\right), \quad \text { with } \quad j=0,1, \ldots, N .
$$


The derivatives of the dependent variables are obtained by differentiating the expansion above, the first derivative for example,

$$
f^{\prime}(z)=\sum_{n=0}^{N} a_{n} T_{n}^{\prime}(z),
$$

the Chebyshev polynomials derivatives can be found using the following relation:

$$
T_{n}^{(d)}\left(z_{j}\right)=2 n, T_{n-1}^{(d-1)}\left(z_{j}\right)+\frac{n}{n-2} T_{n-2}^{(d)}, \quad \text { with } \quad n=3,4, \ldots
$$

where, the subscript $d$ denotes the order of derivatives. For more details about the method the reader is advised to refer to [46].

Next, the Orr-Sommerfeld eigenvalue problem (3.20-3.22) is solved using the scheme described earlier. First, the eigenfunctions $\varphi(y), \tau(y)$, and their derivatives are mapped from the physical domain $(0 \leq y \leq h)$ to the Chebyshev conical domain $(-1 \leq z \leq 1)$ by using the transformation $z=2 y / h-1$ :

$$
\begin{aligned}
\varphi(z) & =\varphi(y), & D^{m} \varphi(z) & =\left(\frac{2}{h}\right)^{m} D^{m} \varphi(y), \\
\tau(z) & =\tau(y), & D^{m} \tau(z) & =\left(\frac{2}{h}\right)^{m} D^{m} \tau(y),
\end{aligned}
$$

where $m$ denotes the order of differentiation. After this mapping is done, the Orr-Sommerfeld eigenvalue problem is rearranged in the following form:

$$
\begin{aligned}
& \left(\frac{4}{h^{2}} D^{2}-k^{2}\right)^{2} \varphi+3 i k \operatorname{Re} U\left(k^{2} \varphi-\frac{4}{h^{2}} D^{2} \varphi\right)+3 i k \operatorname{Re} D^{2} U \varphi=c\left[3 i k \operatorname{Re}\left(k^{2} \varphi-\frac{4}{h^{2}} D^{2} \varphi\right)\right] \\
& \left(\frac{4}{h^{2}} D^{2}-k^{2}\right) \tau+3 \operatorname{RePrik}(D \Theta \varphi-U \tau)=-c[3 i k \operatorname{RePr} \tau]
\end{aligned}
$$

with boundary conditions at the wall

$$
\begin{aligned}
& \varphi(0)=D \varphi(0)=0, \\
& \tau(0)=0
\end{aligned}
$$

and at the free surface

$$
\begin{aligned}
& \varphi(h)+\frac{1}{2} \eta=c \eta, \\
& \begin{aligned}
\left(\frac{4}{h^{2}} D^{2}-\right. & \left.3 k^{2}-3 i k R e \bar{U}\right) \frac{2}{h} D \varphi(h)-i \eta k\left(C t+(W e-M \bar{\Theta}) k^{2}\right) \\
& +i k V_{r}\left(\frac{\tau(h)}{K}+\frac{D \bar{\Theta}}{K} \eta\right)=c\left[-\frac{3}{2 h} i k \operatorname{Re} D \varphi(h)\right],
\end{aligned}
\end{aligned}
$$




$$
\begin{aligned}
& \left(\frac{4}{h^{2}} D^{2}+k^{2}\right) \varphi(h)+i k M\left(\eta \frac{2}{h} D \bar{\Theta}+\tau(h)\right)-\eta=0 \\
& \frac{2}{h} D \tau(1)+\frac{\tau}{K}-\frac{J}{K} \eta=0 .
\end{aligned}
$$

The system of equations 3.42 can be simplified by finding $\eta$ from the tangential boundary condition $3.42 . \mathrm{c})$, and then substitute it in the kinematic $3.42 \mathrm{a}$ ), normal 3.42,b) and energy 3.42, d) boundary conditions [39]. Next the eigenfunctions $\varphi(z)$ and $\tau(z)$ are expanded as truncated series of the Chebyshev polynomials:

$$
\varphi(z)=\sum_{n=0}^{N} a_{n} T_{n}(z), \quad \quad \tau(z)=\sum_{n=0}^{N} b_{n} T_{n}(z) .
$$

The OS eigenvalue problem is written in the generalized matrix eigenvalue problem:

$$
\mathbf{A w}=c \mathbf{B w}
$$

where $\mathbf{A}$ and $\mathbf{B}$ are square matrices of size $2 N+2$, while $\mathbf{w}$ is $2 N+2$ long, where $\mathrm{N}$ is the number of collocation points. The following diagram shows how $\mathbf{A}$ and B matrices are set:

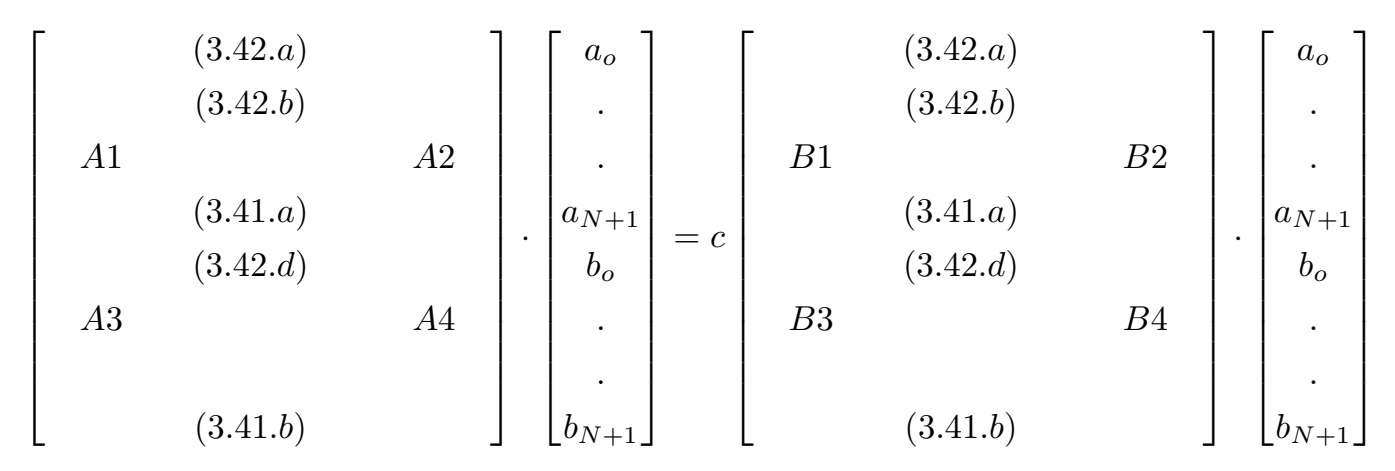

The governing equations are governed by the matrices:

$$
\begin{aligned}
& A 1=\left(D^{2}-k^{2}\right)^{2} \varphi+3 i k \operatorname{Re} U\left(k^{2} \varphi-4 D^{2} \varphi\right)+3 i k \operatorname{Re} D^{2} U \varphi \\
& A 2=0 \\
& A 3=3 \operatorname{RePrikD\Theta \varphi }, \\
& A 4=\left(D^{2}-k^{2}\right) \tau-3 \operatorname{RePrikU\tau }, \\
& B 1=3 i k \operatorname{Re}\left(k^{2} \varphi-4 D^{2} \varphi\right), \\
& B 2=0,
\end{aligned}
$$




$$
\begin{aligned}
& B 3=0, \\
& B 4=3 i k \operatorname{Re} \operatorname{Pr} \tau .
\end{aligned}
$$

where $\mathbf{D}$ denotes the Chebyshev differentiation matrices. The interface boundary conditions for the $\varphi(z)$ are applied on the first two rows as shown, while their order does not not affect the accuracy of the results. Same goes for the energy boundary condition where it is applied on the first rows of (A3, B3, and B4). With regards to the wall boundary conditions, they are applied on the last row of the governing equations matrices, the associated spurious eigenvalues can be removed by multiplying the same rows in matrix $\mathbf{A}$ by a complex multiple of the corresponding rows in B [47]. Another method to apply the wall boundary conditions is to multiply the Chebyshev polynomials by another polynomial such that the two conditions are satisfied at same point [48], both of the methods were applied and the obtained results had an excellent agreement. The eigenvalue problem was solved using the QZ algorithm [49] implemented in MATLAB. The number of collocation points sufficient to obtain accurate results is $15-30$.

\subsection{Model validation}

The derived Orr-Sommerfeld eigenvalue problem including evaporation effects is validated by comparing it against the long wave theory for small $R e$ and $k$, this will also validate the numerical scheme presented earlier. Excellent agreement was found by comparing the results to several references in the literature.

\subsubsection{Non-volatile falling films}

The Orr-Sommerfeld model is first validated for a non-volatile liquid. For small wavenumbers $k$ and weak inertia (small $R e$ ), the results are compared against those of the long wave model derived in section 3.1. Figure 3.1(a) shows the perturbation growth rate $\omega_{i}$ as a function of the Reynolds number Re, when the wavenumber is small $k=0.001$. Excellent match is found between the two models 

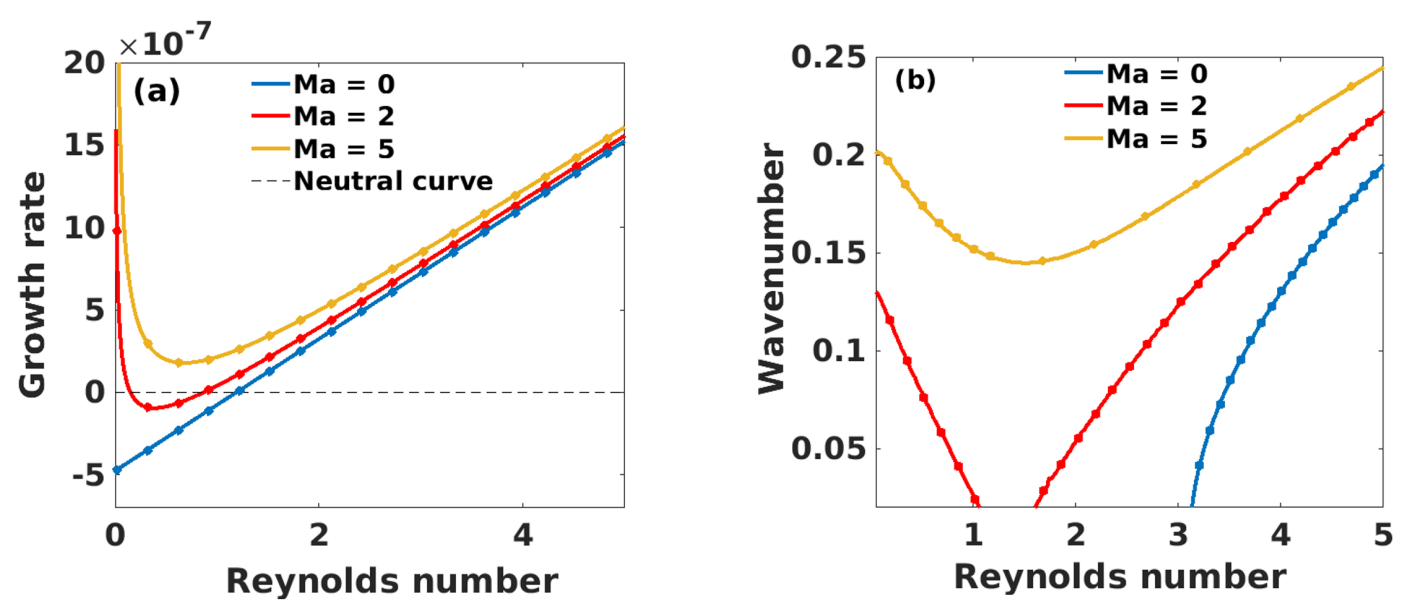

Figure 3.1: (a) Growth rate comparison between the current OS solver (solid line) and LW approximation (circles) (b) Neutral curve comparison between the current OS solver (solid line) and OS eigenvalue problem solved by Kalliadasis et al [1] (circles).

for isothermal case (blue line), and also when the Marangoni effect is added.

The model was also validated for larger wavenumbers $k$ and Reynolds numbers Re by comparing the results to the neutral curves (the line where the flow goes from unustable to stable or the other way around) obtained by Kalliadasis et al. [1], see figure 3.1b. Both results are for the following parameters: $\beta=15^{\circ}$, $K a=250, \operatorname{Pr}=7$, and $B i=1$.

\subsubsection{Volatile falling films}

Since the Orr-Sommerfeld eigenvalue problem has not been derived for a falling film with evaporation present, the only benchmark available to validate our model is by comparing it against the long wave theory. Figure 3.2 , a considers the vapor recoil effect with the absence of mass loss, again the two models agrees very well in the small $(k-R e)$ region for different values of the parameter $\mathcal{V}_{r}$. The results also agree with the long wave theory in terms of mass loss effect with the absence of vapor recoil, as it can be seen from figure 3.2.(b). The growth rate decreases as the film thins. The dry out time $t_{d}$ also increases with Reynolds number $(R e)$. 

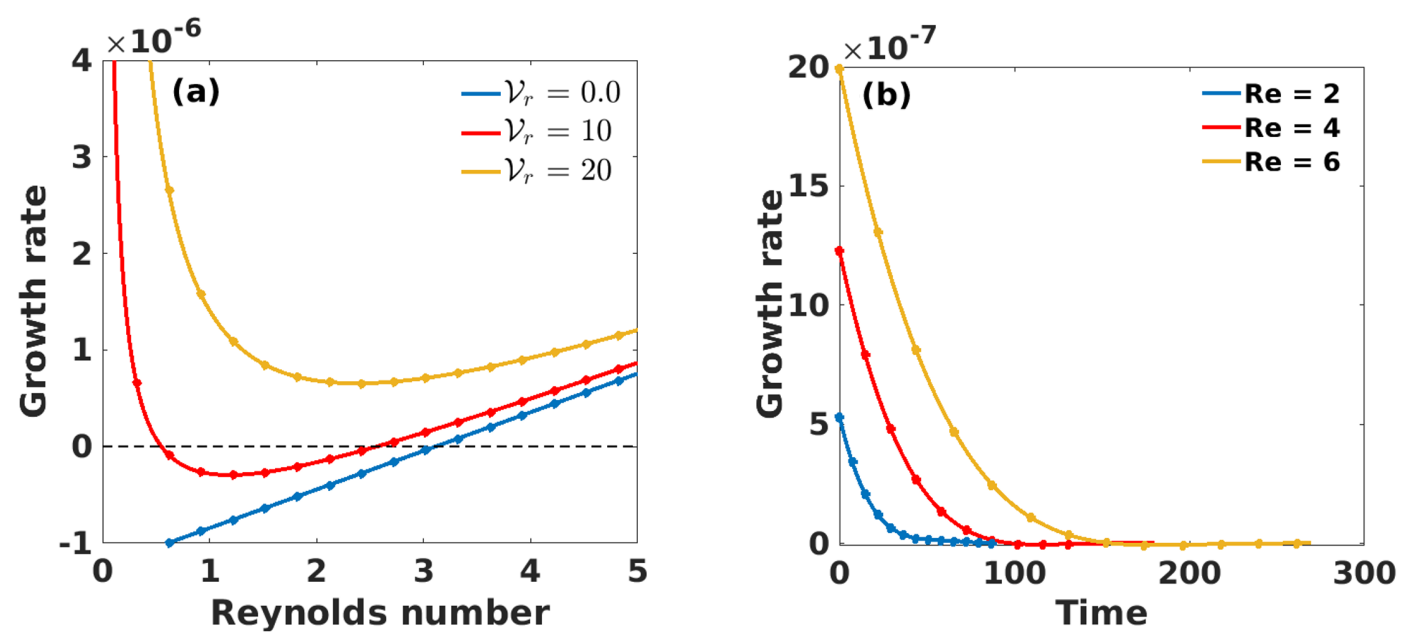

Figure 3.2: Growth rate comparison between the current OS solver for $k=$ 0.001 (solid line) and LW approximation (circles) (a) for different values of $E$ vs Reynolds number and (b) for different values of $R e$ in function of time. 


\section{Chapter 4}

\section{Linear stability analysis: results and discussions}

The different instability modes discussed in this monograph are analyzed through growth rate, and energy perturbations. Section 4.1 is devoted to the hydrodynamic instability (H-mode), while section 4.2 discusses the stability of non-volatile heated films where Marangoni effect is present (S-mode). The main contribution of this work is presented in section 4.3 , where we discuss the instability caused by evaporation effects (E-mode), and how it affects other instability modes. Finally, we present a detailed perturbation energy analysis in section 4.4 .

\subsection{Hydrodynamic instability (H-mode)}

In the absence of any thermal effects, only one unstable mode exists, the well known H-mode [1] shown in figure 4.1. This mode is due to gravity effects induced by surface inclination. For small Reynolds number Re, the inertia is weak and hydrostatic pressure is stabilizing the flow. The inertia becomes stronger as the Reynolds number increases, and at some point it overcomes the hydrostatic pressure and destabilizes the flow at $(R e>5 / 6 C t)$, see figure 4.1(a). 

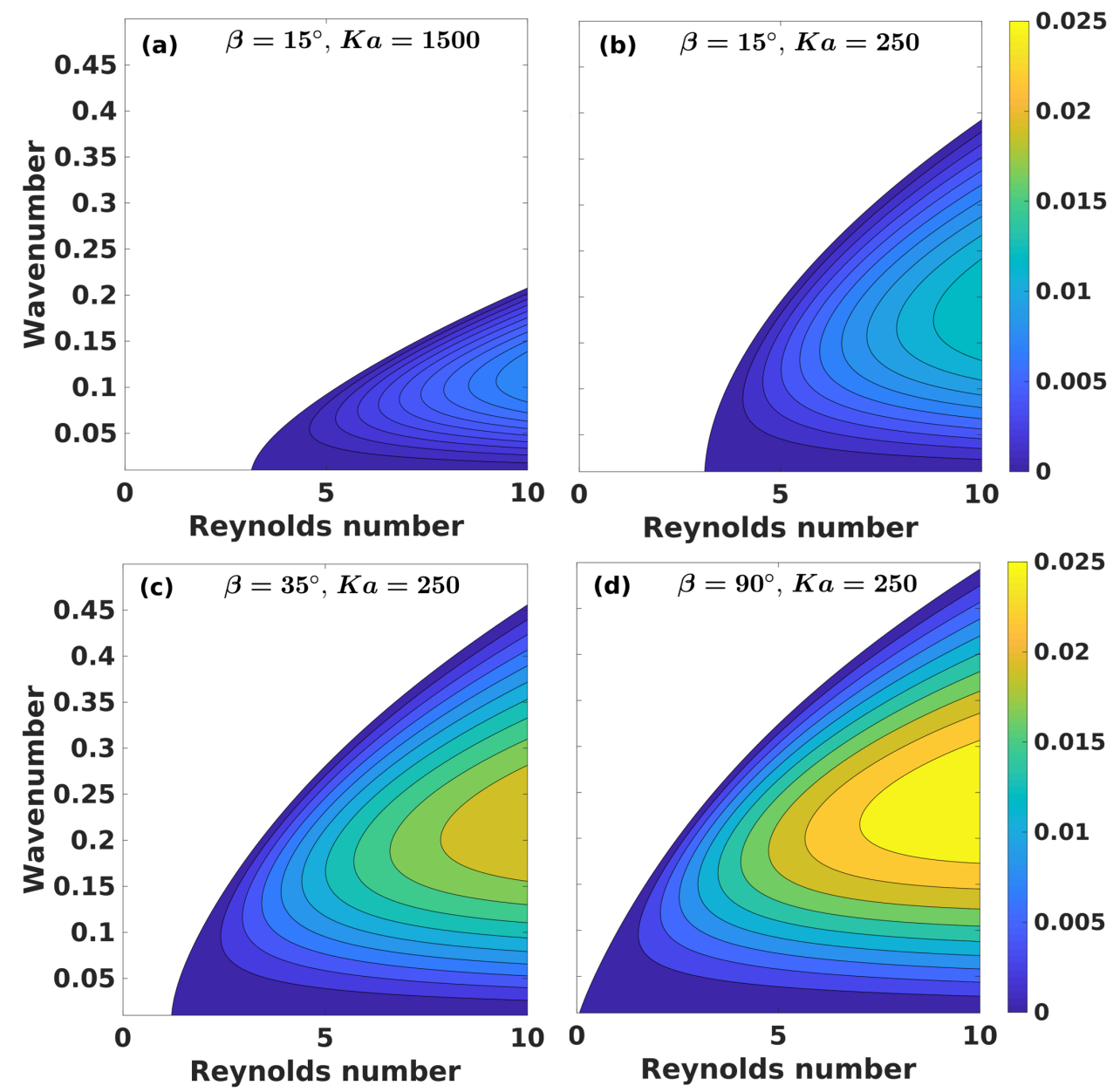

Figure 4.1: Contours of the growth rate in the $R e-k$ plane showing the H-mode for different Kapitza number $K a$ and inclination angle $\beta$.

Figure 4.1(b) shows the effect of decreasing the surface tension force by decreasing the Kapitza number $K a$. The unstable region expands along the wavenumber axis, since short wavelength perturbations are destabilized due decrease in surface tension, while long wavelength perturbations in the limit $(k \rightarrow 0)$ are unaffected showing that the critical number remains the same $(R e>5 / 6 C t)$.

The consequence of changing the inclination angle $\beta$ on the H-mode is easily observed by comparing figures 4.1(a) and 4.1(c). As $\beta$ increases, the destabilizing streamwise film inertia increases, while the stabilizing hydrostatic pressure 
decreases, this yields to expanding the H-mode region, and also to higher growth rates. Additionally, the critical Reynolds number decreases too. We also recovered the classical result by Benjamin [8], where the film is unstable for all Reynolds numbers at $\beta=90^{\circ}$ in the limit $(k \rightarrow 0)$ as shown in 4.1(d).

\subsection{Thermocapillary instability (S-mode)}

Next, thermal effects are taken into consideration, while the liquid is still nonvolatile. As it is possible to see in figure 4.2, for small values of $M a$, a second unstable mode associated with the Marangoni effect appears for small values of $R e$, this mode is called S-mode, which is a result of surface tension changes due to the temperature gradient along the free surface. Additionally, the H-mode is further destabilized, where the critical Reynolds number at which isothermal flows are unstable has decreased, see difference between figures 4.2 (a) and 4.2(b). Moreover, the S-mode disappears as Re increases. More interestingly as $M a$ increases, the two modes combine into one unstable region, thus showing that they can support each other figure $4.2 \mathrm{~b}$.
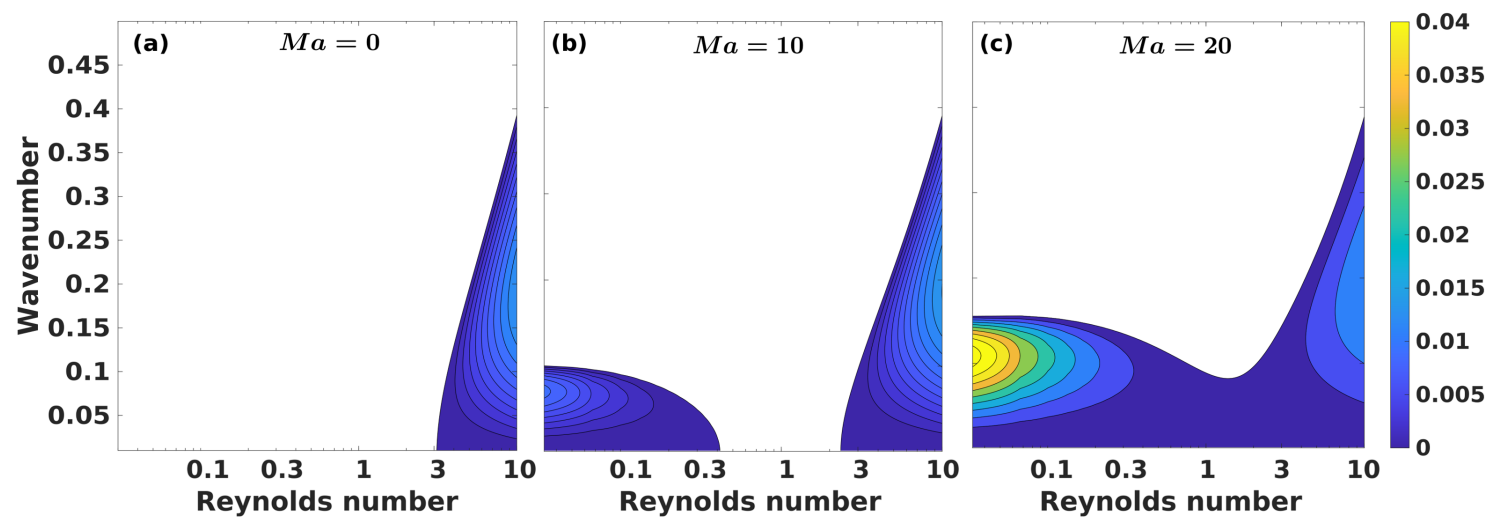

Figure 4.2: Contours of the growth rate in the $(R e-k)$-plane with $K a=250$, $K=1, \operatorname{Pr}=7, \beta=15^{\circ}$, and (a) $M a=0$ (b) $M a=10$ (c) $M a=20$. 


\subsection{Vapor recoil instability (E-mode)}

The results presented here are associated with the evaporation effect on the temporal instability of falling liquid films. First, we compare the effect of vapor recoil between the extended Orr-Sommerfeld eigenvalue problem and the long wave theory for larger wavenumbers $k$. Figure 4.3 shows the temporal growth rate $\omega_{i}$ as a function of $k$ for different values of $R e$. The two models agree when the $k$ is small and inertia is weak $(R e=1)$, however, as $R e$ increases the long wave theory shows huge error in predicting $\omega_{i}$, see $R e=5$ and $R e=15$ for instance. Moreover, the error in predicting the cut-off $k_{c}$ also increases as $R e$ increases. This shows the main contribution of this monograph to the literature, the extended Orr-Sommerfeld model eliminates the restrictions made on the long wave theory and provides wider view of the vapor recoil instability in falling liquid films.

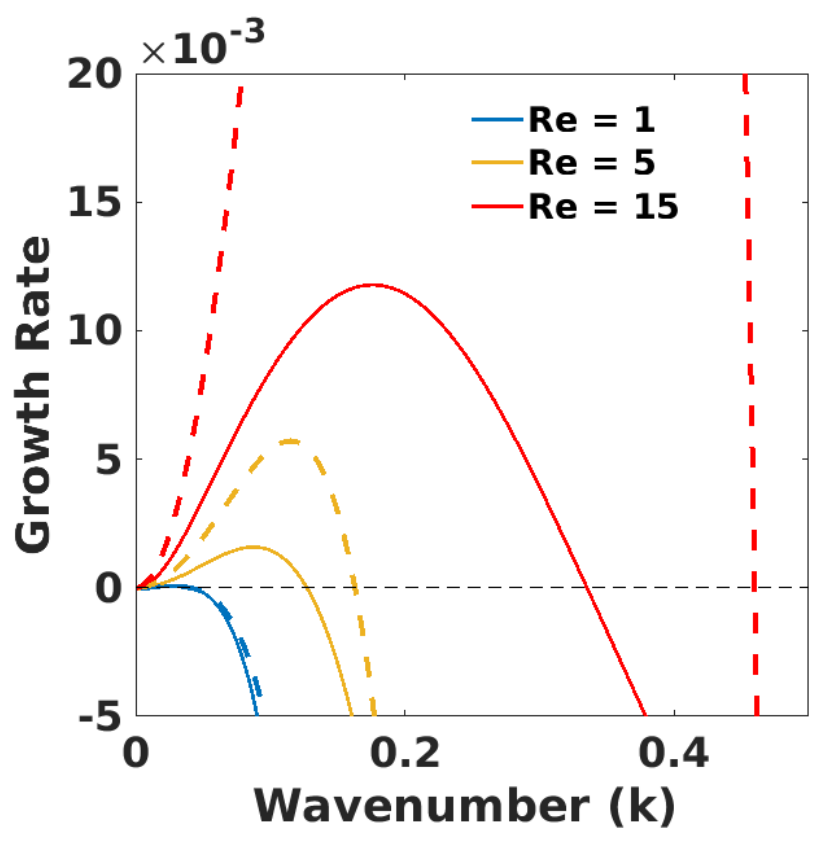

Figure 4.3: Temporal growth rate $\omega_{i}$ in versus the wavenumber $k$. Comparison is made between extended OS model (solid line) and LW expansion (dashed line) for different values of Reynolds number, when $\mathcal{V}_{r}=10, K=1, \beta=15^{\circ}, K a=250$, $\operatorname{Pr}=7$. 

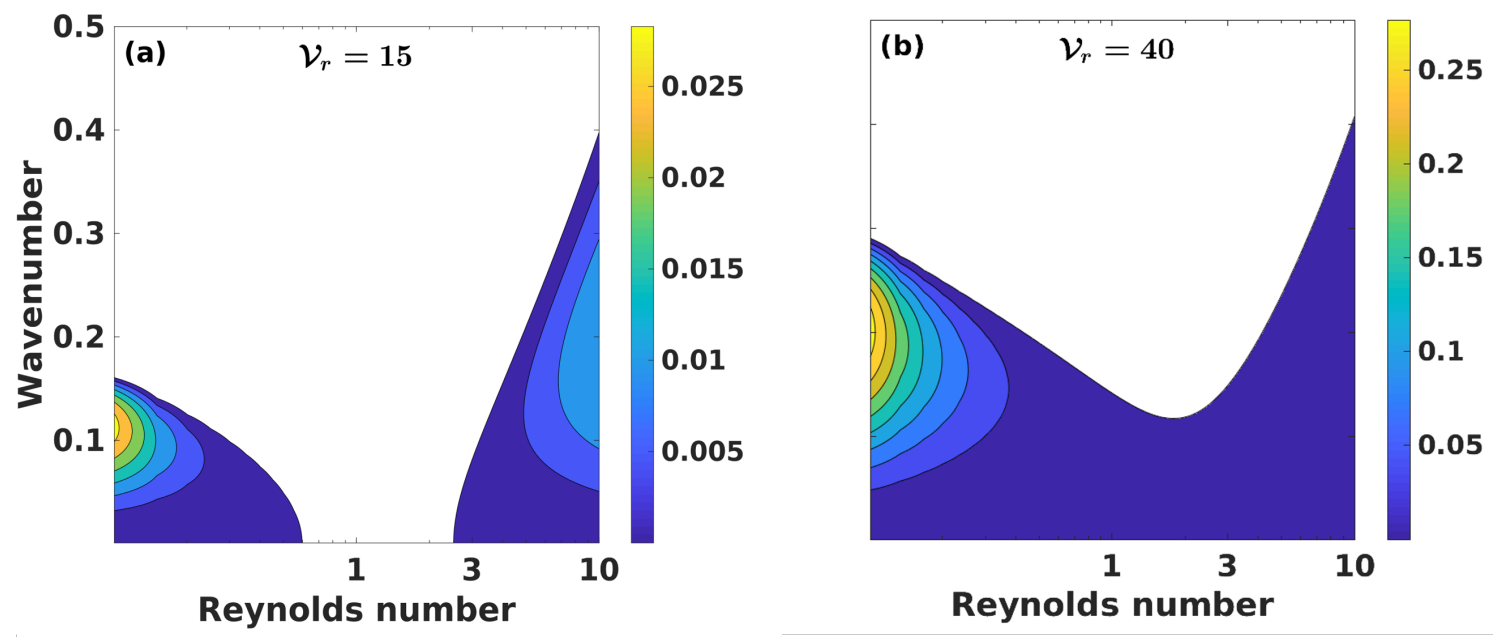

Figure 4.4: Contours of the growth rate in the $R e-k$ plane with $K a=250, \operatorname{Pr}=7$, $\beta=15^{\circ}, K=1$, and (a) $\mathcal{V}_{r}=15$ (b) $\mathcal{V}_{r}=40$

Figure 4.4(a) shows the effect of vapor recoil in the $(R e-k)$ plane. For small $\mathcal{V}_{r}$, a new instability window opens at small $R e$, while the H-mode region is expanded. We named this new instability mode as E-mode. Moreover, as Re increases the E-mode disappears. For larger values of $\mathcal{V}_{r}$, the two modes combine into one unstable mode. Consequently, we conclude that the vapor recoil effect (E-mode) have similar behavior as the Marangoni effect (S-mode).

The combined effect of Marangoni (S-mode) and vapor recoil (E-mode) is studied for two different values of Re. Figure 4.5(a) shows the growth rate along the wavenumber for a small $R e$, the H-mode does not exist, while the green line shows growth rate due to the introduction of Marangoni effect only. Then vapor recoil effect only is introduced to further increase the growth rate (red line). Combining the effects of vapor recoil and Marangoni results in a significant increase in the growth rate, which indicates that Marangoni and evaporation effects enhance each other. For larger Re, Figure 4.5(b) shows how the combined effects enhance each other and the H-mode at the same time, causing a significant increase in the growth rate. 

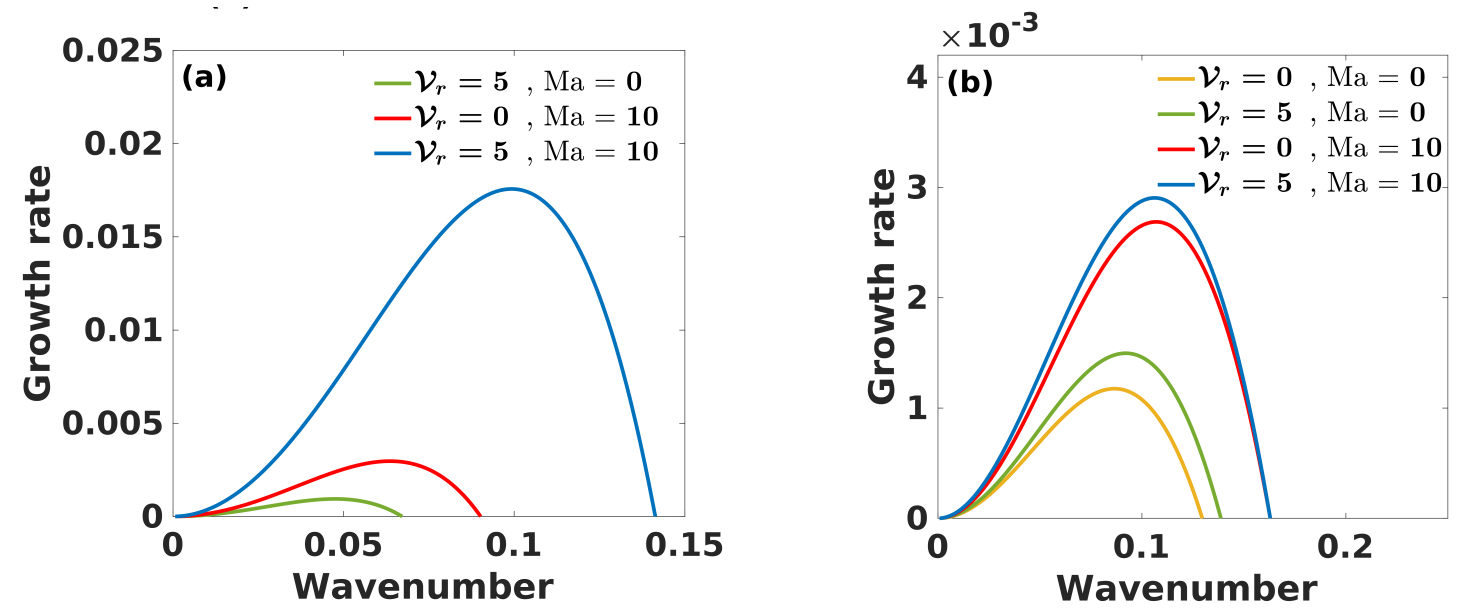

Figure 4.5: Temporal growth rate $\omega_{i}$ in terms of the wavenumber $k$ for two different Reynolds numbers, (a) $R e=0.1$ and (b) $R e=4$. Parameters are different combinations of $\mathcal{V}_{r}$ and $M a$, while $K=1, \beta=15^{\circ}, K a=250, \operatorname{Pr}=7$, $B i=1$.

The effect of vapor recoil without any Marangoni effect is shown in figure 4.6. For $(K=0)$ which is a representative of the quasi-equilibrium case, the temperature gradient across the film is constant (see figure 2.3c), therefore the trough of a wave will experience higher mass fluxes, and hence will have higher vapor recoil than a crest, this in return destabilizes the film. As the parameter $K$ increases, corresponding to the non-equilibrium cases, the film becomes less volatile, and consequently the vapor recoil effect is weaker, thus stabilizing the film.

Finally, the effect of mass loss (film thinning) is examined. If we compare the growth contours for a thinning film in figures 4.7(b) and 4.7(c) against a constant thickness film in figure 4.7(a), we see that the H-mode shrinks as the film thins, while the E-mode is expanding. This is expected because as the film thins, the viscous forces become more dominant and therefore more stabilizing. At the same time, the evaporation rate becomes higher as the film thins, and thus the vapor recoil effect is stronger which expands the E-mode further. 


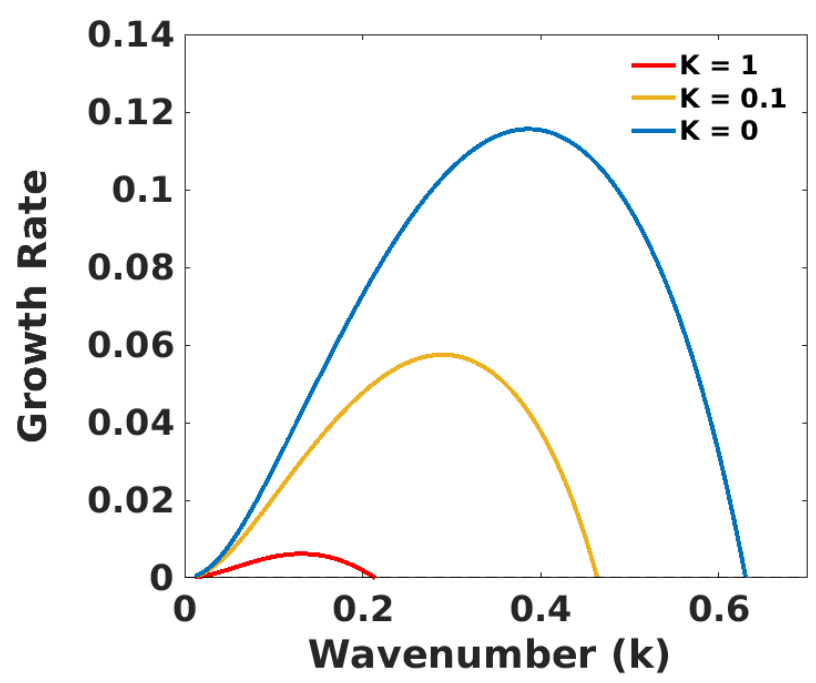

Figure 4.6: Temporal growth rate $\omega_{i}$ in terms of the wavenumber $k$ for different values of the parameter $K$, when $R e=1, \mathcal{V}_{r}=20, \beta=15^{\circ}, K a=250, \operatorname{Pr}=7$, $B i=1$ 

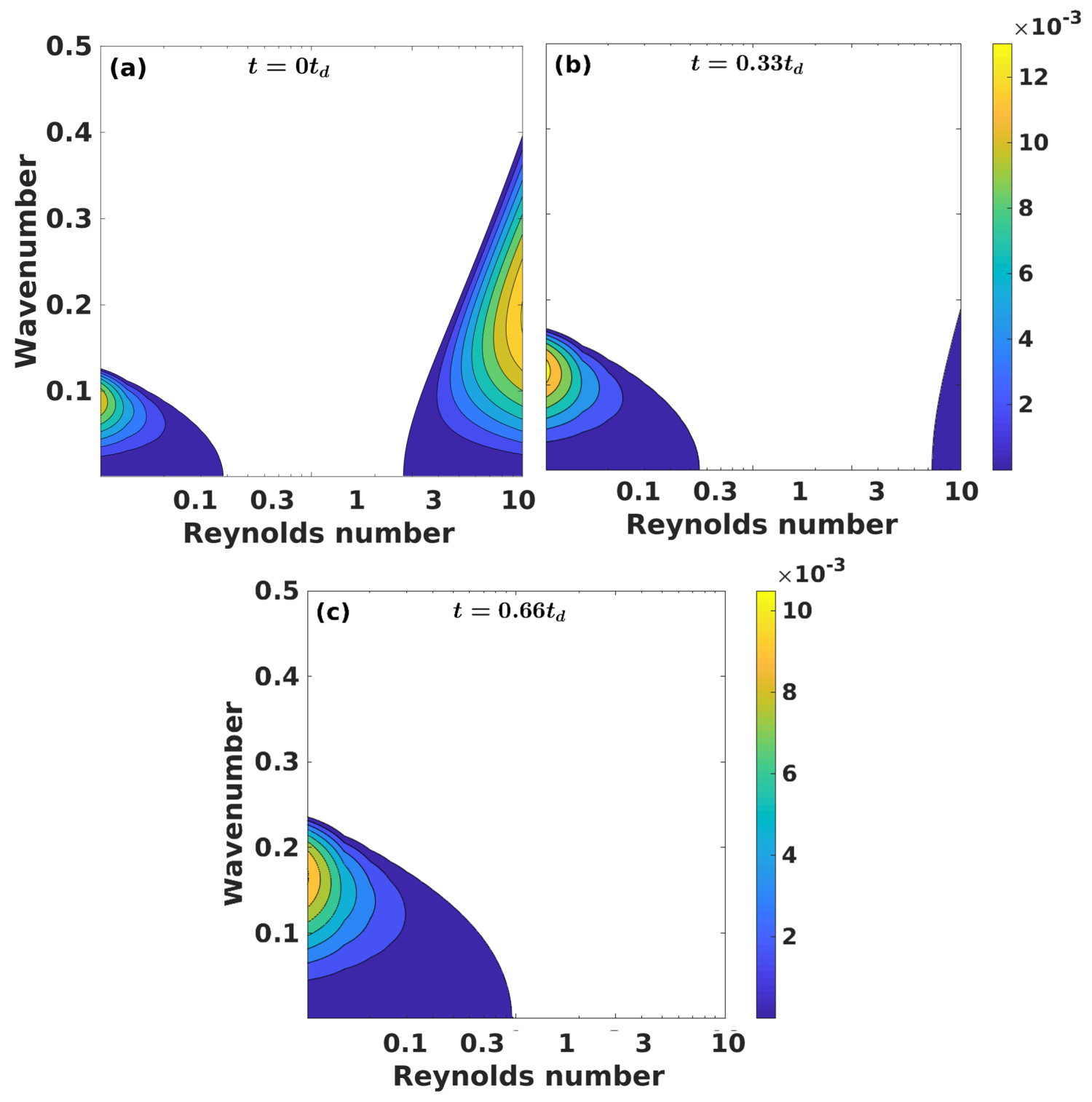

Figure 4.7: Contours of the growth rate in the $R e-k$ plane with $K a=250$, $\operatorname{Pr}=7, \beta=15^{\circ}, K=1$, and $\mathcal{V}_{r}=10$ for times: (a) $t=0 t_{d}$ (b) $t=0.33 t_{d}$ and (c) $t=0.66 t_{d}$ 


\subsection{Perturbation energy}

In order to understand how the instabilities are generated and by which components of the flow, the different terms in the kinetic energy balance represented by equation 3.33 are evaluated. The energy balance gives more insight on the results presented in the previous section and how the different modes of instability enhance each other. First we revisit the energy balance equation for completeness:

$$
K I N+S T E+H Y D=R E Y+S H E+D I S+M A R+V R E
$$

The right hand side terms represent the rate of change of available energy, while the left hand side terms represent the way this energy is distributed to the perturbation. In the following, we distinguish the terms associated with the different instability modes, and also show how instability modes support each other through energy analysis. In all the results presented in this section, asterisks $\left(^{*}\right)$ indicate that the energy terms are normalized with the dissipation energy DIS. In this way, the contribution of every term in the energy balance to the instability modes is more easily examined.

\subsubsection{Energy analysis of the H-mode}
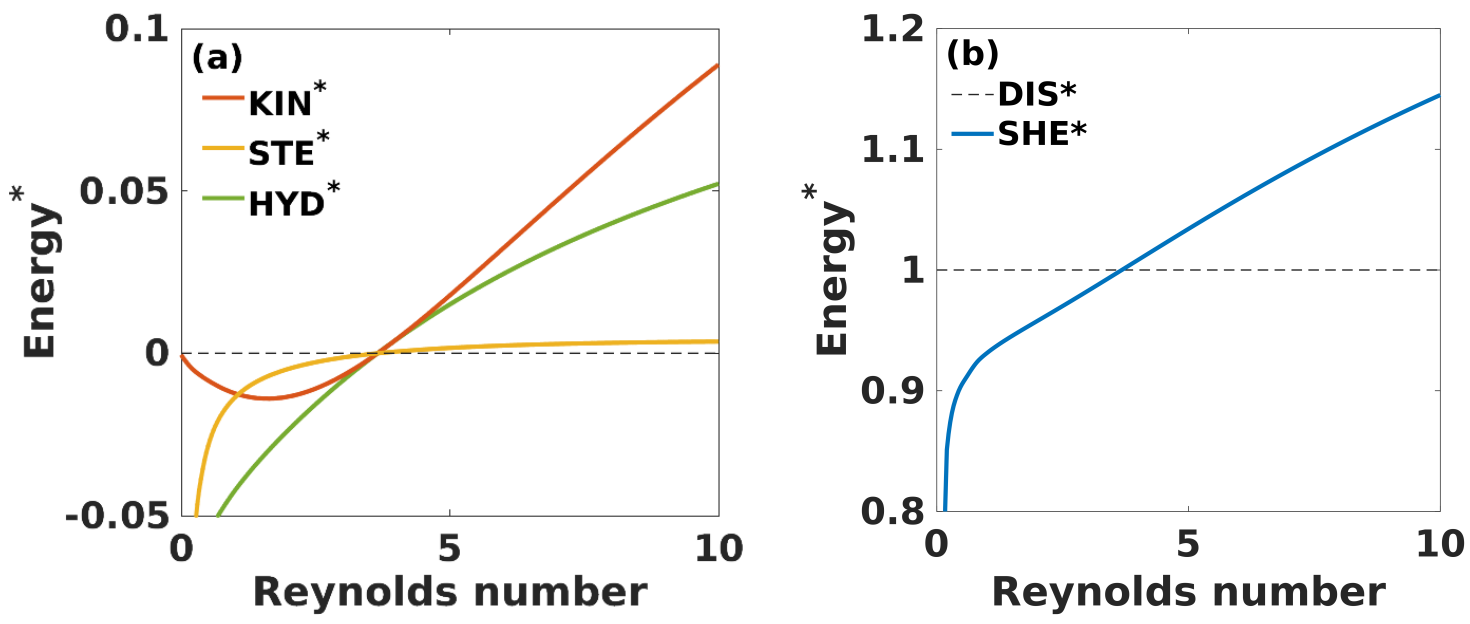

Figure 4.8: Normalized energy terms along $R e$ for $k=0.1, K a=250, \beta=15^{\circ}$ 
Figure 4.8(a) shows the left hand side terms in the kinetic energy balance for $k=0.1$. Generally, they are proportional to the growth rate as mentioned earlier. Moreover, when $R e$ is small the flow rate is low, and HYD is comparable to STE, however as Re increases HYD becomes dominant and STE becomes negligible for such small wavenumber. For an isothermal film, the net available energy comes only from the work done by the perturbation shear stress SHE, the flow is unstable when SHE < DIS, and unstable otherwise. see figure 4.8(b).

\subsubsection{Energy analysis of the S-mode}
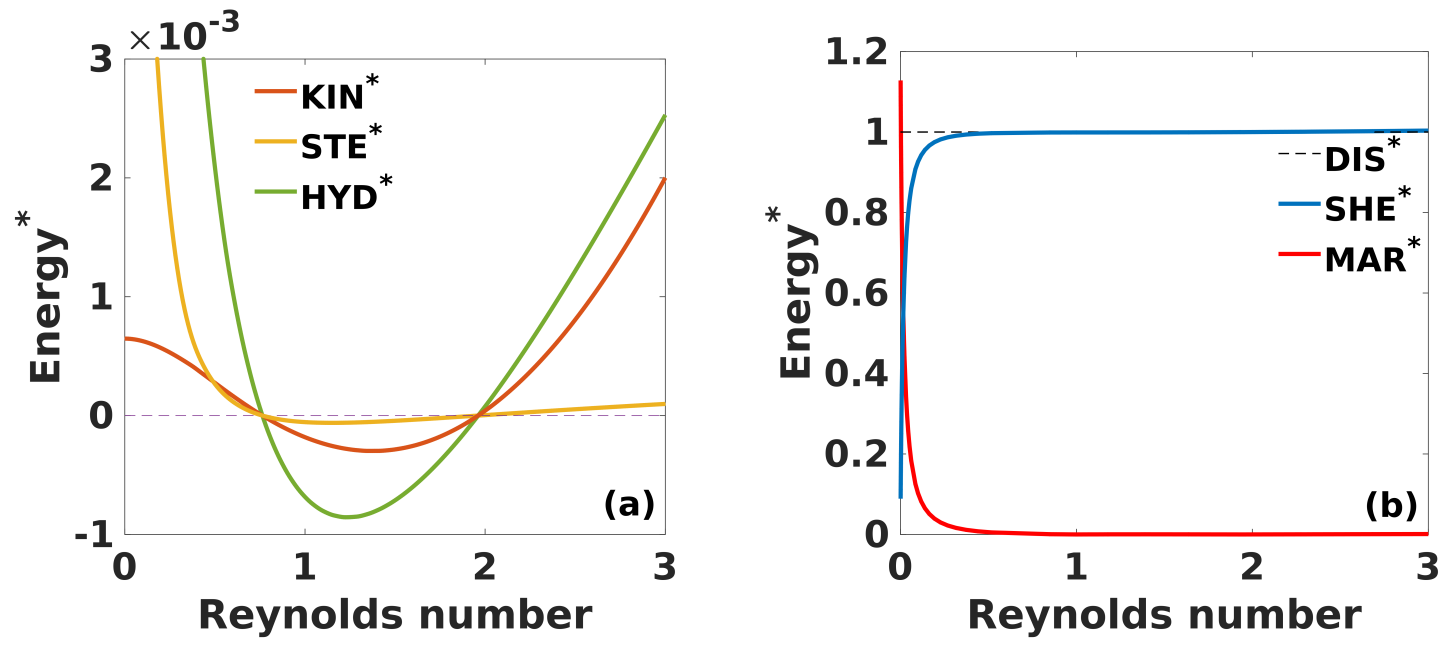

Figure 4.9: Normalized energy terms along $R e$ for $k=0.1, K a=250, K=1$, $\operatorname{Pr}=7, \beta=15^{\circ}$.

Similarly, KIN* ${ }^{*} \mathrm{HYD}^{*}$, and $\mathrm{STE}^{*}$ are positive when the flow is unstable $\left(\omega_{i}>0\right)$, they are positive in the S-mode and H-mode as seen in figure 4.9. (a). However the results shown in 4.9 (b) are more significant, as it compares $\mathrm{SHE}^{*}$ and $\mathrm{MAR}^{*}$ along Re. For $R e<<1, \mathrm{SHE}^{*}$ is negligible, and $\mathrm{MAR}^{*}$ is of $\mathcal{O}(1)$, as $R e$ increases, $\mathrm{SHE}^{*}$ increases and $\mathrm{MAR}^{*}$ decreases. More interestingly, MAR* becomes negligible and $\mathrm{SHE}^{*}$ becomes $\mathcal{O}(1)$ even before the S-mode window closes, indicating that the instability there is due to $\mathrm{SHE}^{*}$. Nevertheless, the instability at small values of Re occurs only when $M a \neq 0$, it is evident that the slight contribution of the S-mode is essential. 


\subsubsection{Energy analysis of the E-mode}

With regarding the vapor recoil effect, we found that the interaction the E-mode interacts with the H-mode the same way as the S-mode. Figure 4.10 shows similar results to those of figure 4.9. Specially, the fact that $\mathrm{VRE}^{*}$ becomes negligible even before the E-mode window closes.
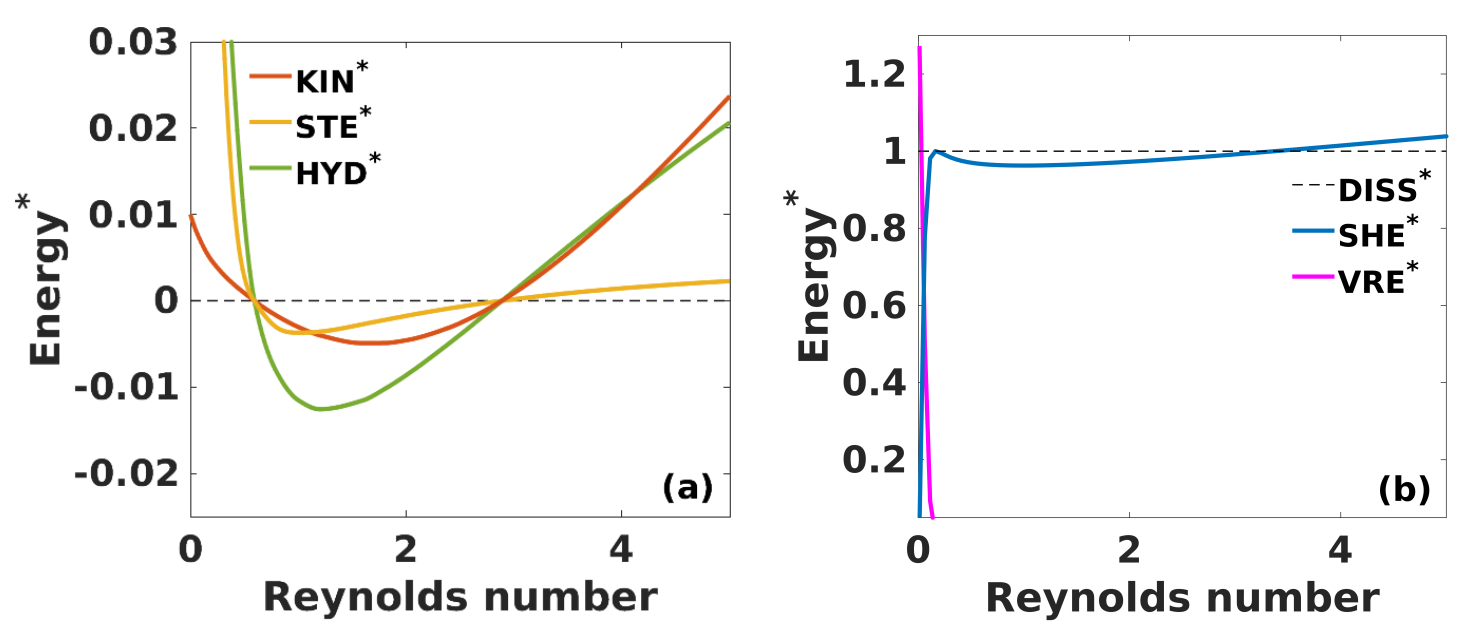

Figure 4.10: Normalized energy terms along $R e$ for $k=0.1, K a=250, \operatorname{Pr}=7$, $\beta=15^{\circ}, M a=0, \mathcal{V}_{r}=10$, and $K=1$.

\subsubsection{Energy analysis of combined S-mode and E-mode}

Figure 4.11(a) emphasis on the fact that the E-mode and S-mode enhance each

other. In order to examine this effect clearly, we plot the terms MAR* and $\mathrm{VRE}^{*}$ as a function of the wavenumber at $R e=0.01$. When $M a=10$ and $\mathcal{V}_{r}=0$, the sum of $\mathrm{SHE}^{*}$ and MAR* creates the unstable S-mode (red line). On the other hand, when $M a=0$ and $\mathcal{V}_{r}=2.5$ the S-mode disappears and the E-mode exists only as a result of the work done by shear stress and vapor recoil $\left(\mathrm{SHE}^{*}+\mathrm{VRE}^{*}\right)$ shown in the blue line. When both Marangoni effect and vapor recoil are present, the total energy, i.e. a combination of work done by the shear stress, thermocapillary and vapor recoil $\left(\mathrm{SHE}^{*}+\mathrm{MAR}^{*}+\mathrm{VRE}^{*}\right)$ increases significantly to a value much larger than for the S-mode or E-mode alone. This 
shows that the two modes enhance each other. An alternative way to show the interaction between the S-mode and E-mode is plotting the terms $\mathrm{SHE}^{*}, \mathrm{MAR}^{*}$ and $\mathrm{VRE}^{*}$ for each of the two modes separated and also when the two modes are combined as shown in figure 4.11. $\mathrm{SHE}^{*}$ is plotted for four different cases as shown in figure 4.11(b), since $R e$ is small no significant changes occur. With regards to MAR*, figure 4.11(c) shows a drastic increase when S-mode is combined with E-mode. Finally, VRE* show a significant increase as well when E-mode is combined with S-mode, as shown in figure 4.11(d). This indicates that E-mode and S-mode enhance each other.
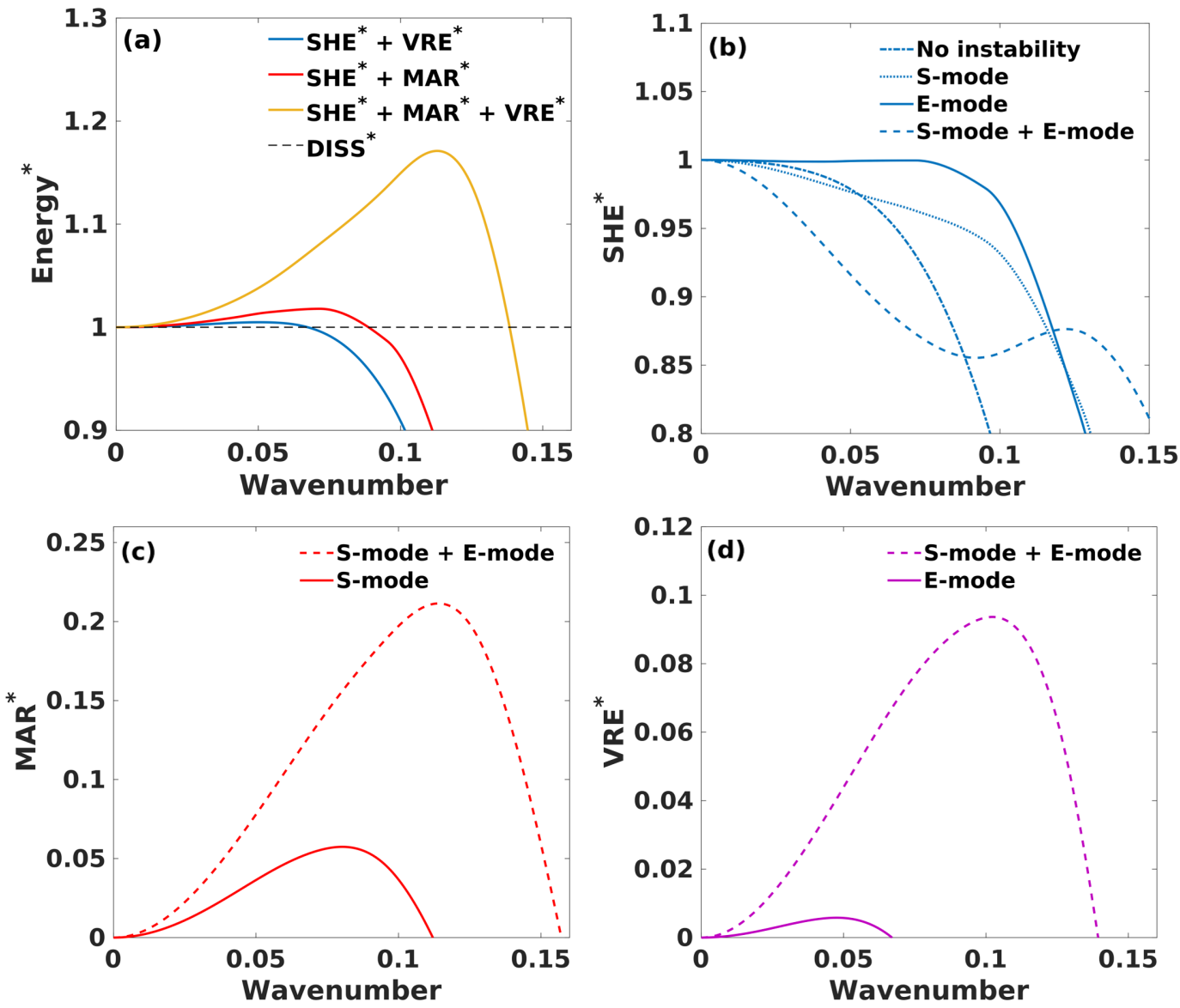

Figure 4.11: Normalized energy terms versus $k$, for $R e=0.1, \beta=15^{\circ}, K a=250$, $\operatorname{Pr}=7, K=1$, (a) total energy when $M a=10$ and $\mathcal{V}_{r}=0$ (red line), $M a=0$ and $\mathcal{V}_{r}=5$ (blue line), $M a=10$ and $\mathcal{V}_{r}=5$ (yellow line). (b) SHE*, (c) MAR*, and (d)VRE* 


\section{Chapter 5}

\section{Conclusions and perspectives}

In summary, we extended the Orr-Sommerfeld eigenvalue problem to include evaporation effects in falling liquid films. We also demonstrated how the long wave theory, which is the only available model in the literature, is only valid for small Reynolds numbers and wavenumbers. We also reassured that the E-mode induced by vapor recoil effect behaves the same way as the S-mode induced by Marangoni effect, we also showed that the flow is most unstable due to these modes when Reynolds number is small. The degree of equilibrium of evaporation was considered as well, where it was found to be highly effective. We also studied the interaction between the well known hydrodynamic instability mode (H-mode) and the E-mode induced by vapor recoil. It was found that the H-mode is enhanced by the E-mode the same way as it is enhanced by the S-mode induced by Maranogni effect. Moreover, we showed that the S-mode and E-mode enhance each other, specially when Reynolds number is small.

In order to understand which mechanisms contribute the most to the evaporation instability E-mode, we extended the perturbation kinetic energy balance to include evaporation effects, an extra term corresponding to the work done by vapor recoil was added. We found that, the work done by vapor recoil is necessary to have the E-mode, however, when Reynolds number increases, it does not contribute to the instability as much as the work done by perturbation shear 
stress. We also found that the work done by Marangoni effect is significantly enhanced when vapor recoil is present, while the opposite holds as well, which indicates that the two modes highly enhance one another.

Moreover, studying the temporal instability of falling liquid films using direct numerical simulations (DNS) was part of this work. A front tracking/finite volume solver for multiphase flows was adapted to simulate isothermal falling liquid films. The solver is based on the one-fluid approach, where the governing equations are solved for the whole computational domain. We validated the DNS solver by comparing the root mean square velocity $\left(U_{r m s}\right)$ against the temporal growth rate obtained from the OS solution, and excellent agreement was found, see appendix C for more details. Furthermore, the energy equation was incorporated in the DNS solver to account for the heat transfer between the liquid and the gas. Adding the Marangoni effect is a part of our future plans, which can be done easily by calculating the temperature at the interface, and accounting for the gradient in temperature when calculating the surface tension. Nevertheless, evaporation effects will also be accounted for in the DNS solver by implementing the model derived by Muradoglu [54] for evaporating process in multiphase flows. Having Marangoni and evaporation effects implemented in the DNS solver allow us to accurately study the linear and nonlinear instability of evaporating falling liquid films without the aid of approximated models, which could be of a great addition to the literature.

Finally, there are several possible ways to extend the theoretical work presented in this thesis. An analytical solution of the different terms in the kinetic energy balance can be obtained by assuming a long wave instability. This will provide simple expressions to examine the perturbation energy without the need for numerical solvers. In addition, the Orr Sommerfeld eigenvalue problem can be used to study the evaporation instability in the transverse direction. It can also be utilized to study the impact of evaporation effects on the short-wave instability modes such as thermocapillary P-mode. Moreover, a simple sign adjustment to the OS model can transform it to study the condensation effect of the instability of falling liquid films. 


\section{Bibliography}

[1] S. Kalliadasis, C. Ruyer-Quil, B. Scheid, and M. G. Velarde, Falling liquid films, vol. 176. Springer Science \& Business Media, 2011.

[2] G. Tryggvason, R. Scardovelli, and S. Zaleski, Direct Numerical Simulations of Gas-Liquid Multiphase Flows. Cambridge University Press, 2011.

[3] P. Colinet, J. C. Legros, and M. G. Velarde, Nonlinear dynamics of surfacetension-driven instabilities. Wiley-vch, 2001.

[4] S. L. Goren and R. Mani, "Mass transfer through horizontal liquid films in wavy motion," AIChE Journal, vol. 14, no. 1, pp. 57-61, 1968.

[5] D. P. Frisk and E. J. Davis, "The enhancement of heat transfer by waves in stratified gas-liquid flow," International Journal of Heat and Mass Transfer, vol. 15, no. 8, pp. 1537-1552, 1972.

[6] M. K. Smith, "The mechanism for the long-wave instability in thin liquid films," Journal of Fluid Mechanics, vol. 217, pp. 469-485, 1990.

[7] C.-S. Yih, "Stability of liquid flow down an inclined plane," The physics of Fluids, vol. 6, no. 3, pp. 321-334, 1963.

[8] T. B. Benjamin, "Wave formation in laminar flow down an inclined plane," Journal of Fluid Mechanics, vol. 2, no. 6, pp. 554-573, 1957.

[9] R. Kelly, D. Goussis, S. Lin, and F. Hsu, "The mechanism for surface wave instability in film flow down an inclined plane," Physics of Fluids A: Fluid Dynamics, vol. 1, no. 5, pp. 819-828, 1989. 
[10] K. Smith, "On convective instability induced by surface-tension gradients," Journal of Fluid Mechanics, vol. 24, no. 2, pp. 401-414, 1966.

[11] D. Benney, "Long waves on liquid films," Journal of mathematics and physics, vol. 45, no. 1-4, pp. 150-155, 1966.

[12] S. P. Lin, "Finite-amplitude stability of a parallel flow with a free surface," Journal of Fluid Mechanics, vol. 36, no. 1, p. 113-126, 1969.

[13] A. Pumir, P. Manneville, and Y. Pomeau, "On solitary waves running down an inclined plane," Journal of Fluid Mechanics, vol. 135, p. 27-50, 1983.

[14] S. Saprykin, E. A. Demekhin, and S. Kalliadasis, "Self-organization of twodimensional waves in an active dispersive-dissipative nonlinear medium," Physical review letters, vol. 94, no. 22, p. 224101, 2005.

[15] A. Oron and O. Gottlieb, "Subcritical and supercritical bifurcations of the first-and second-order benney equations," Journal of engineering mathematics, vol. 50, no. 2-3, pp. 121-140, 2004.

[16] C. Ruyer-Quil and P. Manneville, "Modeling film flows down inclined planes," The European Physical Journal B-Condensed Matter and Complex Systems, vol. 6, no. 2, pp. 277-292, 1998.

[17] B. Scheid, C. Ruyer-Quil, and P. Manneville, "Wave patterns in film flows: modelling and three-dimensional waves," Journal of Fluid Mechanics, vol. 562, pp. 183-222, 2006.

[18] R. Craster and O. Matar, "Dynamics and stability of thin liquid films," Reviews of modern physics, vol. 81, no. 3, p. 1131, 2009.

[19] P. Kapitza and S. Kapitza, "Wavy flow of thin layers of viscous fluid," Collected papers of PL Kapitza, vol. 2, pp. 662-709, 1965.

[20] V. Y. Shkadov, "Wave flow regimes of a thin layer of viscous fluid subject to gravity," Fluid Dynamics, vol. 2, no. 1, pp. 29-34, 1967. 
[21] C. Ruyer-Quil and P. Manneville, "Improved modeling of flows down inclined planes," The European Physical Journal B-Condensed Matter and Complex Systems, vol. 15, no. 2, pp. 357-369, 2000.

[22] C. Ruyer-Quil and P. Manneville, "Further accuracy and convergence results on the modeling of flows down inclined planes by weighted-residual approximations," Physics of Fluids, vol. 14, no. 1, pp. 170-183, 2002.

[23] B. Gjevik, "Occurrence of finite-amplitude surface waves on falling liquid films," The Physics of fluids, vol. 13, no. 8, pp. 1918-1925, 1970.

[24] S. Lin, "Finite amplitude side-band stability of a viscous film," Journal of Fluid Mechanics, vol. 63, no. 3, pp. 417-429, 1974.

[25] S. Sreenivasan and S. Lin, "Surface tension driven instability of a liquid film flow down a heated incline," International Journal of Heat and Mass Transfer, vol. 21, no. 12, pp. 1517-1526, 1978.

[26] R. Kelly, S. H. Davis, and D. A. Goussis, "On the instability of heated film flow with variable surface tension," in International Heat Transfer Conference Digital Library, Begel House Inc., 1986.

[27] S. G. Bankoff, "Stability of liquid flow down a heated inclined plane," International Journal of Heat and Mass Transfer, vol. 14, no. 3, pp. 377-385, 1971.

[28] B. Spindler, "Linear stability of liquid films with interfacial phase change," International Journal of Heat and Mass Transfer, vol. 25, no. 2, pp. 161-173, 1982.

[29] J. P. Burelbach, S. G. Bankoff, and S. H. Davis, "Nonlinear stability of evaporating/condensing liquid films," Journal of Fluid Mechanics, vol. 195, pp. 463-494, 1988.

[30] S. Joo, S. H. Davis, and S. Bankoff, "Long-wave instabilities of heated falling films: two-dimensional theory of uniform layers," Journal of Fluid Mechanics, vol. 230, pp. 117-146, 1991. 
[31] A. Oron, "Three-dimensional nonlinear dynamics of thin liquid films," Physical review letters, vol. 85, no. 10, p. 2108, 2000.

[32] A. Oron, "Nonlinear dynamics of three-dimensional long-wave marangoni instability in thin liquid films," Physics of Fluids, vol. 12, no. 7, pp. 1633$1645,2000$.

[33] H. J. Palmer, "The hydrodynamic stability of rapidly evaporating liquids at reduced pressure," Journal of Fluid Mechanics, vol. 75, no. 3, p. 487-511, 1976.

[34] J.-M. Delhaye, "Jump conditions and entropy sources in two-phase systems. local instant formulation," International Journal of Multiphase Flow, vol. 1, no. 3, pp. 395-409, 1974.

[35] W. Nusselt, "The surface condensation of water vapour," VDI Z, vol. 60, pp. 541-546, 1916.

[36] R. Atherton and G. Homsy, "On the derivation of evolution equations for interfacial waves," Chemical Engineering Communications, vol. 2, no. 2, pp. 57-77, 1976.

[37] W. B. Krantz and S. Goren, "Stability of thin liquid films flowing down a plane," Industrial \&5 Engineering Chemistry Fundamentals, vol. 10, no. 1, pp. 91-101, 1971.

[38] S. G Yiantsios and B. Higgins, "Rayleigh-taylor instability in thin viscous films," Physics of Fluids A Fluid Dynamics, vol. 1, p. 1484, 091989.

[39] D. Goussis and R. Kelly, "Surface wave and thermocapillary instabilities in a liquid film flow," Journal of Fluid Mechanics, vol. 223, pp. 25-45, 1991.

[40] R. Vellingiri, D. Tseluiko, and S. Kalliadasis, "Absolute and convective instabilities in counter-current gas-liquid film flows," Journal of Fluid Mechanics, vol. 763, pp. 166-201, 2015.

[41] T. W. Kao, "Stability of two-layer viscous stratified flow down an inclined plane," The Physics of Fluids, vol. 8, no. 5, pp. 812-820, 1965. 
[42] B. E. Anshus and S. L. Goren, "A method of getting approximate solutions to the orr-sommerfeld equation for flow on a vertical wall," AIChE Journal, vol. 12, no. 5, pp. 1004-1008, 1966.

[43] A. Davey, "A simple numerical method for solving orr-sommerfeld problems," The Quarterly Journal of Mechanics and Applied Mathematics, vol. 26, no. 4, pp. 401-411, 1973.

[44] G. De Bruin, "Stability of a layer of liquid flowing down an inclined plane," Journal of Engineering Mathematics, vol. 8, no. 3, pp. 259-270, 1974.

[45] S. A. Orszag, "Accurate solution of the orr-sommerfeld stability equation," Journal of Fluid Mechanics, vol. 50, no. 4, pp. 689-703, 1971.

[46] L. Trefethen, Spectral Methods in MATLAB. Software, Environments, and Tools, Society for Industrial and Applied Mathematics, 2000.

[47] P. J. Schmid and D. S. Henningson, Stability and transition in shear flows, vol. 142. Springer Science \& Business Media, 2012.

[48] L. N. Trefethen, Spectral methods in MATLAB, vol. 10. Siam, 2000.

[49] C. B. Moler and G. W. Stewart, "An algorithm for generalized matrix eigenvalue problems," SIAM Journal on Numerical Analysis, vol. 10, no. 2, pp. 241-256, 1973.

[50] G. Tryggvason, "A front-tracking/finite-volume navier-stokes solver for direct numerical simulations of multiphase flows," October, vol. 19, pp. 11-30p, 2012.

[51] G. Tryggvason, B. Bunner, A. Esmaeeli, D. Juric, N. Al-Rawahi, W. Tauber, J. Han, S. Nas, and Y.-J. Jan, "A front-tracking method for the computations of multiphase flow," Journal of computational physics, vol. 169, no. 2, pp. 708-759, 2001.

[52] A. J. Chorin, "Numerical solution of the navier-stokes equations," Mathematics of computation, vol. 22, no. 104, pp. 745-762, 1968. 
[53] G. Tryggvason, R. Scardovelli, and S. Zaleski, Direct numerical simulations of gas-liquid multiphase flows. Cambridge University Press, 2011.

[54] M. Irfan and M. Muradoglu, "A front tracking method for direct numerical simulation of evaporation process in a multiphase system," Journal of Computational Physics, vol. 337, pp. 132-153, 2017. 


\section{Appendix A}

\section{Interface boundary conditions of the one sided model}

First we present the liquid-vapor jump conditions derived by ref. [34 as follows:

- Mass balance jump condition

$$
J=\rho\left(\mathbf{v}-\mathbf{v}^{(s)}\right) \cdot \mathbf{n}=\rho^{(v)}\left(\mathbf{v}^{(v)}-\mathbf{v}^{(I)}\right) \cdot \mathbf{n},
$$

where, $\rho^{(v)}$ is the vapor density, the subscripts $(v)$ and $(s)$ correspond to vapor and interface quantities, respectively and $\mathbf{n}$ is the unit vector normal to the interface.

- Normal stress jump condition

$$
J\left(\mathbf{v}-\mathbf{v}^{(s)}\right) \cdot \mathbf{n}-\left(\mathbf{P}-\mathbf{P}^{(s)}\right) \cdot \mathbf{n} \cdot \mathbf{n}=2 H \sigma(T),
$$

where $J$ is the mass flux across the interface, $\mathbf{P}$ is the deviatoric stress tensor, and $H$ is the mean curvature of the interface.

- Tangential stress jump condition

$$
J\left(\mathbf{v}-\mathbf{v}^{(s)}\right) \cdot \tau-\left(\mathbf{P}-\mathbf{P}^{(s)}\right) \cdot \mathbf{n} \cdot \tau=-\nabla \sigma \cdot \tau,
$$

where $\nabla_{\mathbf{s}} \sigma$ is the tangential surface tension force, and $\tau$ is the tangential unit vector. 
- Energy jump condition

$$
\begin{array}{r}
J\left[L+\frac{1}{2}\left[\left(\mathbf{v}^{(v)}-\mathbf{v}^{(s)}\right) \cdot \mathbf{n}\right]^{2}-\frac{1}{2}\left[\left(\mathbf{v}-\mathbf{v}^{(s)}\right) \cdot \mathbf{n}\right]^{2}\right]+k \nabla T \cdot \mathbf{n}-k \nabla T^{(v)} \cdot \mathbf{n} \\
+2 \mu(\tau \cdot \mathbf{n}) \cdot\left(\mathbf{v}-\mathbf{v}^{(s)}\right)-2 \mu^{(v)}\left(\tau^{(v)} \cdot \mathbf{n}\right) \cdot\left(\mathbf{v}^{(v)}-\mathbf{v}^{(s)}\right)=0
\end{array}
$$

with $L$ the latent heat of vaporization, $\tau$ and $\tau^{(v)}$ are the rate of deformation stress tensors in the liquid and vapor, respectively.

- No slip at the interface between liquid and vapor

$$
\left(\mathbf{v}-\mathbf{v}^{(s)}\right) \cdot \tau=0 .
$$

We now derive the one sided model based on the assumption that the density, viscosity and thermal conductivity of the liquid are much larger than those of the vapor, we take the following limits:

$$
\frac{\rho^{(v)}}{\rho} \rightarrow 0, \quad \frac{\mu^{(v)}}{\mu} \rightarrow 0, \quad \frac{k^{(v)}}{k} \rightarrow 0 .
$$

Then, we substitute the mass jump balance equation (A.1) in the normal stress jump balance in order to express the velocities in terms of density and mass flux, then by applying the limits we get:

$$
-\frac{J^{2}}{\rho^{(v)}}-(\mathbf{P} \cdot \mathbf{n}) \cdot \mathbf{n}=2 H \sigma(T) .
$$

With regards to the tangential stress jump condition, the first term on the left hand side is removed by substituting the no-slip condition equation (A.5). Then we apply the limits and get:

$$
(\mathbf{P} \cdot \mathbf{n}) \cdot \tau=\nabla_{\mathbf{s}} \sigma \cdot \tau
$$

Finally, the energy jump condition is simplified by applying the limits:

$$
J\left[L+\frac{1}{2}\left[\frac{J}{\rho^{(v)}}\right]^{2}\right]=-k \nabla \mathbf{T} \cdot \mathbf{n} .
$$




\section{Appendix B}

\section{About the Perturbations}

The perturbation quantities, namely $\tilde{u}, \tilde{v}, \tilde{T}, \eta, \mathcal{J}$ are needed for perturbation energy analysis. First we derive the perturbation velocities $\tilde{u}$ and $\tilde{v}$, the perturbation stream function is written as follow:

$$
\psi(x, y, t)=\varphi(y) e^{i(\mathbf{k} \cdot \mathbf{x}-\omega t)}
$$

while the perturbation velocities are:

$$
\begin{gathered}
\tilde{v}=-\partial_{x} \psi(x, y, t)=-i k \varphi(y) e^{i(\mathbf{k} \cdot \mathbf{x}-\omega t)} \\
\tilde{u}=\partial_{y} \psi(x, y, t)=D \varphi(y) e^{i(\mathbf{k} \cdot \mathbf{x}-\omega t)}
\end{gathered}
$$

However, we are only interested in the real part of the velocities, thus we find the real part of the streamfunction as:

$$
\operatorname{Real}(\psi)=\frac{1}{2}\left(\psi+\psi^{*}\right)
$$

where $\psi^{*}$ is the complex conjugate. In order to split the real part from the imaginary part, we expand the streamfunction and it's conjugate as follows:

$$
\begin{aligned}
\psi & =\left(\varphi_{r}+i \varphi_{i}\right) e^{i k\left(x-\left(c_{r} t+i c_{i} t\right)\right)}, \\
\psi^{*} & =\left(\varphi_{r}-i \varphi_{i}\right) e^{i k\left(x-\left(c_{r} t-i c_{i} t\right)\right)} .
\end{aligned}
$$


Next, we write the complex exponential function in term of trigonometric functions using Euler's formula, then we rearrange:

$$
\begin{aligned}
\psi & =\left(\varphi_{r} \cos (\theta)+i \varphi_{r} \sin (\theta)+i \varphi_{i} \cos (\theta)-\varphi_{i} \sin (\theta)\right) Q, \\
\psi^{*} & =\left(\varphi_{r} \cos (\theta)-i \varphi_{r} \sin (\theta)-i \varphi_{i} \cos (\theta)-\varphi_{i} \sin (\theta)\right) Q,
\end{aligned}
$$

where $Q=e^{k c_{i} t}$ and $\theta=k\left(x-c_{r} t\right)$. Next, we get the real part of the stream function as follows:

$$
\begin{gathered}
\operatorname{Real}(\psi)=\frac{1}{2}\left(\psi+\psi^{*}\right), \\
\operatorname{Real}(\psi)=\left(\varphi_{r} \cos (\theta)-\varphi_{i} \sin (\theta)\right) .
\end{gathered}
$$

Finally, the real part of the perturbation velocities is:

$$
\begin{gathered}
\tilde{v}=-\partial_{x} \psi(x, y, t)=k\left(\varphi_{i} \cos (\theta)+\varphi_{r} \sin (\theta)\right) Q, \\
\tilde{u}=\partial_{y} \psi(x, y, t)=\left(D \varphi_{r} \cos (\theta)-D \varphi_{i} \sin (\theta)\right) Q .
\end{gathered}
$$

In a similar manner to how we derived $\tilde{u}$ and $\tilde{v}$, the real part of the perturbation amplitude $\tilde{h}$ is derived as follows:

$$
\begin{aligned}
\tilde{h}(x, t) & =\eta e^{i(\mathbf{k} \cdot \mathbf{x}-\omega t)}, \\
\operatorname{Real}(\tilde{h}) & =\frac{1}{2}\left(\tilde{h}+\tilde{h}^{*}\right),
\end{aligned}
$$

where $\tilde{h}$ and $\tilde{h}^{*}$ are defined as follows:

$$
\begin{aligned}
& \tilde{h}=\left(\eta_{r} \cos (\theta)+i \eta_{r} \sin (\theta)+i \eta_{i} \cos (\theta)-\eta_{i} \sin (\theta)\right) Q, \\
& \tilde{h}^{*}=\left(\eta_{r} \cos (\theta)-i \eta_{r} \sin (\theta)-i \eta_{i} \cos (\theta)-\eta_{i} \sin (\theta)\right) Q .
\end{aligned}
$$

The real part of the perturbation amplitude is:

$$
\tilde{h}=\left(\eta_{r} \cos (\theta)-\eta_{i} \cos (\theta)\right) Q,
$$

where the constant $\eta_{r}$ and $\eta_{i}$ are obtained from the kinematic boundary condition (3.22, a):

$$
\eta=\frac{\varphi_{r}+i \varphi_{i}}{c_{r}+i c_{i}-\bar{U}}
$$


By multiplying by the conjugate of the denominator and rearrange, we get:

$$
\eta=\frac{\left.\varphi_{r}\left(c_{r}-\bar{U}\right)+\varphi_{i} c_{i}\right)}{\left(c_{r}-\bar{U}\right)^{2}+c_{i}^{2}}+i \frac{\left.\varphi_{i}\left(c_{r}-\bar{U}\right)+\varphi_{r} c_{i}\right)}{\left(c_{r}-\bar{U}\right)^{2}+c_{i}^{2}}
$$

with $\eta_{r}$ as the real part, and $\eta_{i}$ as the imaginary part.

The perturbation temperature $\tilde{T}$ is derived using the same approach, the final expression for the real part of $\tilde{T}$ is shown below:

$$
\tilde{T}=\left(\tau_{r} \cos (\theta)-\tau_{i} \cos (\theta)\right) Q
$$




\section{Appendix C}

\section{Direct numerical simulations (DNS) of isothermal films}

This section briefly summarizes the work devoted to direct numerical simulations (DNS) of isothermal falling liquid films. First we briefly present a front tracking/finite volume solver adapted to simulate falling liquid films. Next, the DNS solver is validated for isothermal falling films against linear stability analysis.

\section{C.1 Numerical Solver}

A front tracking/finite volume solver for multiphase flows [50] was adapted to simulate falling liquid films. The solver is based on a single fluid approach [51, where the two phases are treated as one fluid but with different material properties. The governing equations are solved for the whole computational domain on a uniform staggered grid, with the horizontal velocity $u$ is defined at the middle of the right and left edges of the control volume, while the vertical velocity $v$ is defined at the middle of the top bottom, the pressure is defined at the center of the control volume, see figure C.1. 


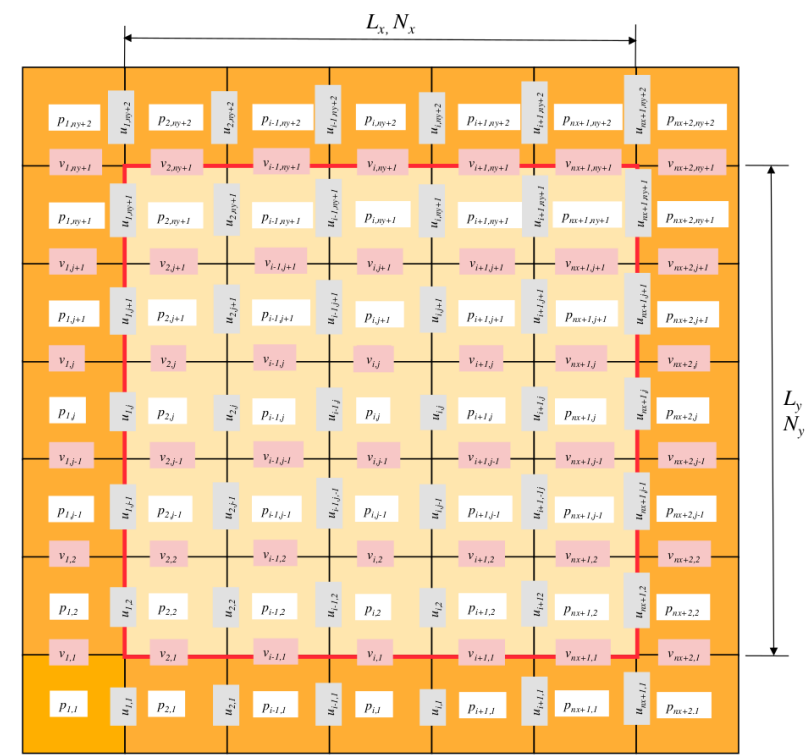

Figure C.1: The standard staggered grid. The pressure nodes reside at the center of control volume. The velocity is stored at the nodes residing in the control volume edges. Picture taken from G. Tryggvason et al. [2].

A second order central difference scheme is used to discretize all the spatial derivatives in the momentum equations, while the time integration is performed using a first-order projection method [52], where a non-free divergence velocity (temporary velocity) field is obtained first without considering the pressure. Afterwards, the pressure is obtained by solving the Poisson equation using a RedBlack Gauss-Seidel method [51]. Finally, the temporary velocity and pressure are used to obtain the corrected velocity at the next time step.

The interface between the two phases is tracked explicitly using a Lagrangian grid. The interface is represented by elements which are connected by marker points. As the interface moves, the liquid and gas domains change, in order to track the two domains, an indicator function $F I(x, t)$ is formed as follows:

$$
F I(x, t)= \begin{cases}1, & \text { in liquid phase } \\ 0, & \text { in gas phase. }\end{cases}
$$


$F I(x, t)$ is updated every time step, then it is used to update the material properties in gas and liquid as follows:

$$
\rho=\rho_{l} F I(x, t)+\rho_{g}(1-F I(x, t)), \quad \mu=\mu_{l} F I(x, t)+\mu_{g}(1-F I(x, t)),
$$

Finally, the connection between the fixed grid and the moving interface is highly important. Surface tension is found at the interface first, then smoothed onto the grid in order to account for in the momentum equation, on the contrary, the velocity is interpolated from the grid onto the interface marker points in order to move the interface. A comprehensive description of this smoothing and interpolating process is available in [53].

\section{C.2 Isothermal falling liquid film simulation}

The temporal stability of falling liquid films was studied using the DNS solver illustrated earlier. In this section we conduct two-dimensional simulations. The domain was represented by a rectangle, with length equal to $L_{x}=\frac{2 \pi}{k}$ in which $k$ is the chosen wavenumber. The bottom edge corresponds to the inclined surface wit a no-slip boundary condition applied there, while an open boundary condition was applied on the top, periodic boundary conditions were set for the sides. The initial conditions are a combination of the base state solution obtained in equation (2.21) plus an initial disturbance on the interface.

$$
h(x)=1+A \sin (k x) .
$$

The amplitude of the disturbance $A$ is approximated as $1 \%$ of initial film height. The disturbance is added in order to trigger the instability of the film, for a stable configuration, the disturbance will fade away with time and the flow will converge to the base state solution. On the other hand, for an unstable flow, the disturbance grows linearly at the beginning, followed by the non-linear region, which finally leads to the rupture of the film.

The DNS tool was verified by comparing the root mean square velocity $\left(U_{r m s}\right)$ with the growth rate obtained from the linear stability analysis. $U_{r m s}$ is calculated 
every time step for the liquid phase only as follows,

$$
U_{r m s}=\left[\int_{0}^{L_{x}} \int_{0}^{L_{y}}(u(x, y, t)-U(y))^{2} d y d x\right]^{\frac{1}{2}}
$$

where $U$ is the initial velocity field.

Figure (C.2) shows that the growth rate predicted by our OS solver perfectly agrees with the evolution of the instability found by DNS, while long wave theory failed to predict the correct growth rate. We obtained a similar result also for different parameters and then we conclude that our 2D isothermal DNS solver is accurate.

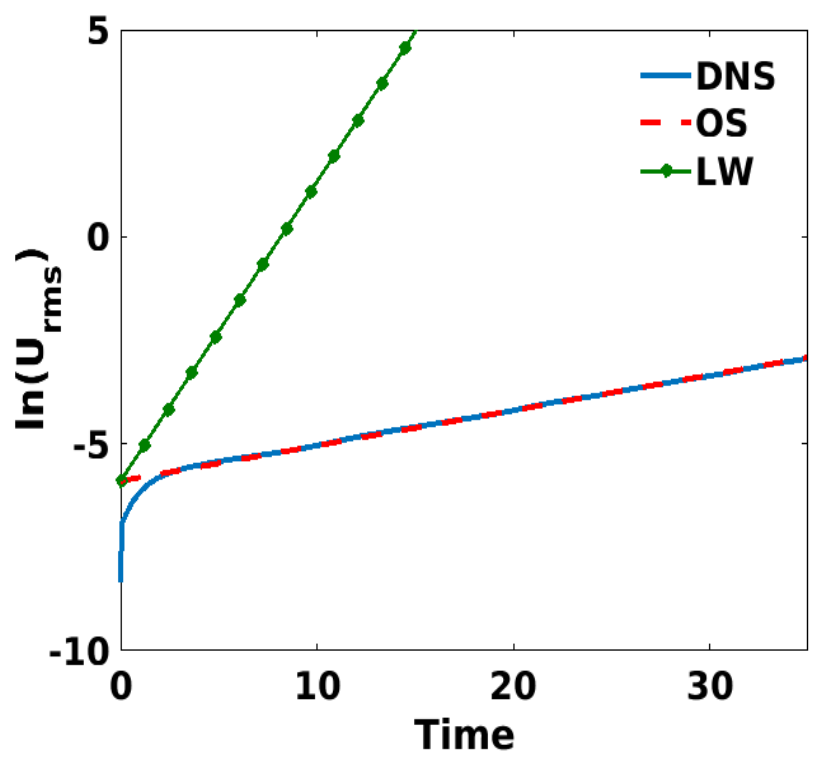

Figure C.2: Logarithm of the root mean square velocity $\ln \left(U_{r m s}\right)$ versus time for $R e=6.5, K a=10, \beta=45^{\circ}$ and $k=0.48$. The linear growth found by our DNS (cyan line) perfectly agrees with the value of the growth rate $\left(\omega_{i}=0.034\right)$ found with our OS solver (dashed red line) while the LW theory (green line) fails to predict it.

Figure (C.3) shows the contours of the horizontal velocity (a,c,e) and the vertical velocity $(\mathrm{b}, \mathrm{d}, \mathrm{f})$ taken from a direct numerical simulation of an unstable isotheraml film falling down an inclined surface. Figures $\overline{C .3} \mathrm{a}$ and $\overline{\mathrm{C} .3 \mathrm{~b}}$ show 
the initial conditions (base state solution with a perturbation of amplitude $\mathrm{A}=$ 0.01). Figures C.3 and C.3 $\mathrm{d}$ show the linear growth of the disturbance, while the figures C.3 and C.3F show the non-linear growth which is characterized by the so-called Kapitza waves [1]. 

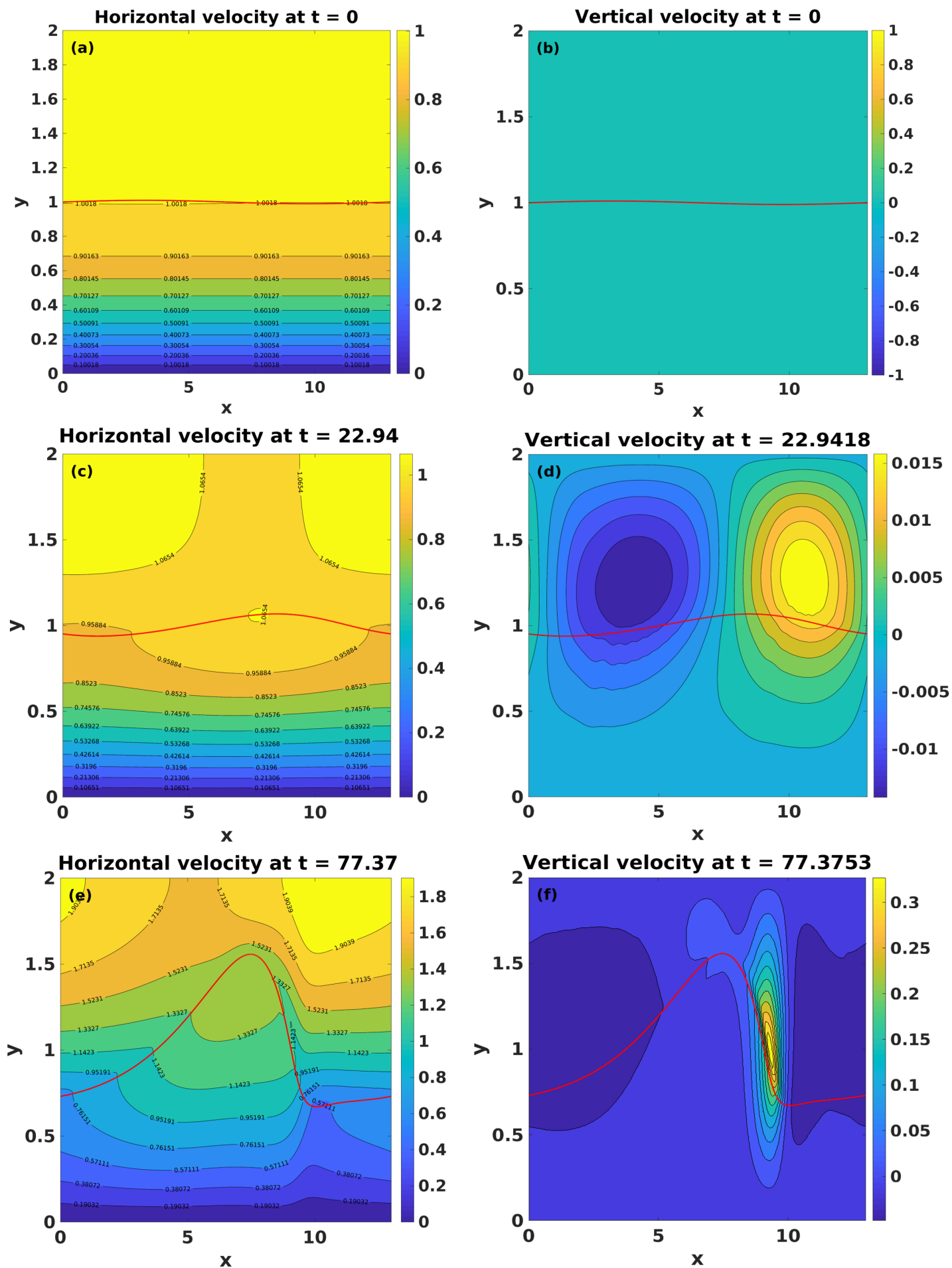

Figure C.3: Contours of the horizontal (a,c,e) and vertical (b,d,f) velocity at different time $(\mathrm{a}, \mathrm{b}) t=0,(\mathrm{c}, \mathrm{d}) t=22.94$ and $(\mathrm{e}, \mathrm{f}) t=77.35$. We consider an isothermal film falling down an inclined surface with $R e=6.5, K a=10, \beta=45^{\circ}$ and $k=0.48$. 